\title{
Ultrasound Elastography and Characterization of Soft Tissue Mimicking Phantoms
}

\author{
By
}

\section{Rachel Morehouse}

\author{
A thesis submitted to \\ the Faculty of Graduate Studies and Research \\ in partial fulfilment of the degree requirements of
}

\section{Master of Applied Science}

Ottawa-Carleton Institute of Electrical and Computer Engineering

Department of Systems and Computer Engineering

Carleton University

Ottawa, Ontario, Canada

(September 2010)

Copyright $\odot$ Rachel Morehouse, 2010 


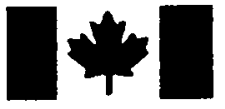

Library and Archives

Canada

Published Heritage

Branch

395 Wellington Street

Ottawa ON K1A ON4

Canada
Bibliotheque et

Archives Canada

Direction du

Patrimoine de l'édition

395 , rue Wellington

Ottawa ON K1A ON4

Canada
Your file Votre reférence

ISBN: 978-0-494-71572-7

Our file Notre référence

ISBN: 978-0-494-71572-7
NOTICE:

The author has granted a nonexclusive license allowing Library and Archives Canada to reproduce, publish, archive, preserve, conserve, communicate to the public by telecommunication or on the Internet, loan, distribute and sell theses worldwide, for commercial or noncommercial purposes, in microform, paper, electronic and/or any other formats.

The author retains copyright ownership and moral rights in this thesis. Neither the thesis nor substantial extracts from it may be printed or otherwise reproduced without the author's permission.
AVIS:

L'auteur a accordé une licence non exclusive permettant à la Bibliothèque et Archives Canada de reproduire, publier, archiver, sauvegarder, conserver, transmettre au public par télécommunication ou par l'Internet, prêter, distribuer et vendre des thèses partout dans le monde, à des fins commerciales ou autres, sur support microforme, papier, électronique et/ou autres formats.

L'auteur conserve la propriété du droit d'auteur et des droits moraux qui protège cette thèse. $\mathrm{Ni}$ la thèse ni des extraits substantiels de celle-ci ne doivent être imprimés ou autrement reproduits sans son autorisation.
In compliance with the Canadian Privacy Act some supporting forms may have been removed from this thesis.

While these forms may be included in the document page count, their removal does not represent any loss of content from the thesis.
Conformément à la loi canadienne sur la protection de la vie privée, quelques formulaires secondaires ont été enlevés de cette thèse.

Bien que ces formulaires aient inclus dans la pagination, il n'y aura aucun contenu manquant. 
The undersigned recommend to

the Faculty of Graduate Studies and Research

acceptance of the thesis

\title{
Ultrasound Elastography and Characterization of Soft Tissue Mimicking Phantoms \\ Submitted by Rachel Morehouse
}

\begin{abstract}
In partial fulfilment of the requirements for the degree of
Master of Applied Science
\end{abstract}

Y. Ono, Thesis Supervisor

H. Schwartz

Chair, Department of Systems and Computer Engineering

Carleton University

2010 


\section{Abstract}

Ultrasound elastography, a noninvasive method of measuring the elastic properties of soft tissues, was investigated using a correlation technique. Phantoms, used in elastography research to mimic biological soft tissue, were fabricated to resemble a tumor using graphite powder as scatterers and two different agar concentrations, 2-w\% and 3-w\% agar for the background and tumor, respectively. Strain images were constructed and evaluated using their SNR and contrast. A multicompression technique reduced decorrelation noise and a 2D median filter removed outlier data points, and thus the imaging quality was improved.

Ultrasound velocity, stress-relaxation, Young's modulus and hysteresis were also investigated using various single concentration agar-based phantoms. The ultrasound velocity of the developed phantoms in the range of the agar concentration between $1 \mathrm{w} \%$ and $3 \mathrm{w} \%$ was $1490 \mathrm{~m} / \mathrm{s} \pm 1 \mathrm{~m} / \mathrm{s}$. The stress-relaxation curves showed that the phantoms gradually relaxed under constant compression. The Young's modulus was $89 \mathrm{kPa}$ and $111 \mathrm{kPa}$ for the tumor background of the tumor phantom, respectively. Hysteresis, where load and unload stress-strain relationships are not the same, was observed for the 2-w\% agar phantom. 


\section{Acknowledgements}

I would like to thank my supervisor Dr. Yuu Ono, Associate Professor of Systems and Computer Engineering, Carleton University for his expertise, guidance and encouragement. I appreciate both the time and effort that he provided me with throughout my graduate studies. I would also like to thank Dr. Andy Adler, Professor of Systems and Computer Engineering, Carleton University for the use of the ultrasound imaging system.

I would also like to express my appreciation to Carleton University and the Natural Sciences and Engineering Research Council of Canada (NSERC) for the opportunity and the financial support that they provided for this research. Also, the

Ontario Graduate Scholarship in Science and Technology and the Allan Buchanan Graduate Scholarship is acknowledged.

I am grateful to my colleagues Jason Silver and George Tanev for their efforts and assistance throughout my research work. Finally, a special thanks to family and friends for their support, enthusiasm and patience. 


\section{Table of Contents}

$\begin{array}{ll}\text { Abstract } & i\end{array}$

Acknowledgements $\quad$ ii

Table of Contents $\quad$ iii

List of Tables v v v v v v

List of Figures $\quad v i$

List of Symbols $\quad v i$

\begin{tabular}{l|l} 
Introduction & $I$
\end{tabular}

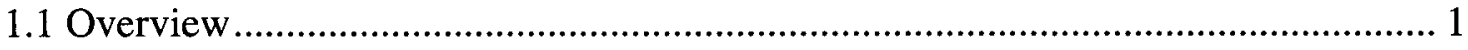

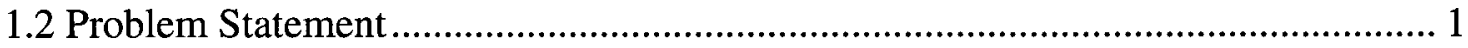

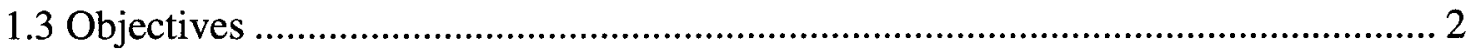

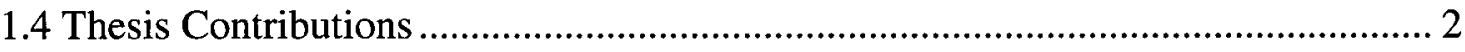

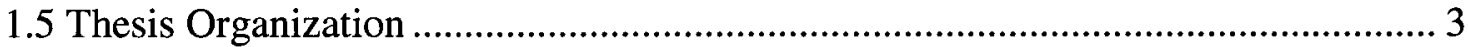

$\begin{array}{ll}\text { Chapter 2: Background Review } & 5\end{array}$

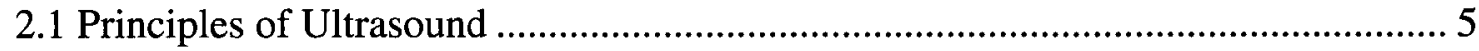

2.1.1 Ultrasound Physics............................................................................ 5

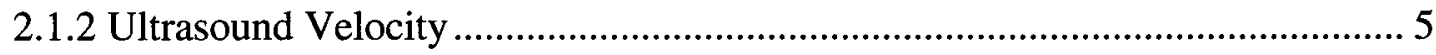

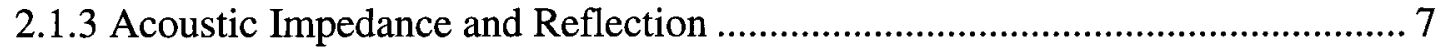

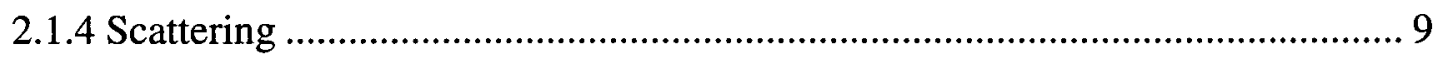

2.2 Methods of Determining Tissue Stiffness.................................................. 10

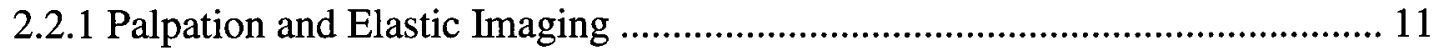


2.3 Tissue Mimicking Phantom Characterization........................................................... 13

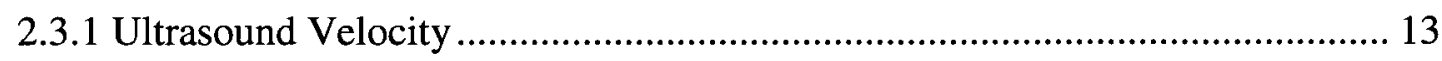

Chapter 3: Tissue Mimicking Phantom Construction and Characterization 16

3.1 Tissue Mimicking Phantom Construction .......................................................... 16

3.2 Tissue Mimicking Phantom Characterization Methods............................................. 21

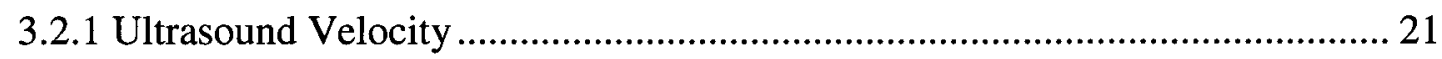

3.2.2 Stress-Relaxation ........................................................................................ 26

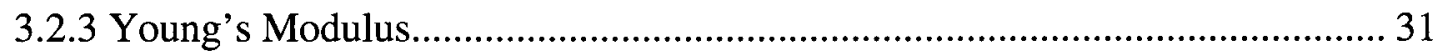

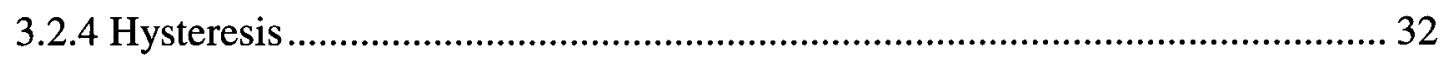

3.3 Tissue Mimicking Phantom Characterization Results and Discussion.................... 34

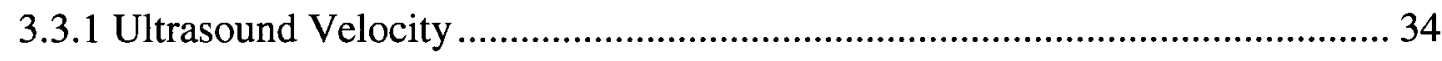

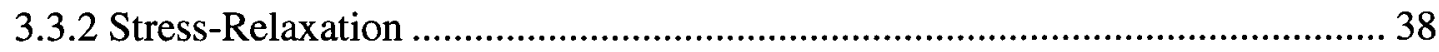

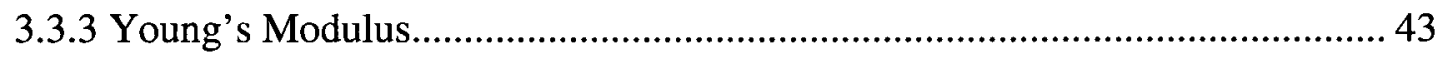

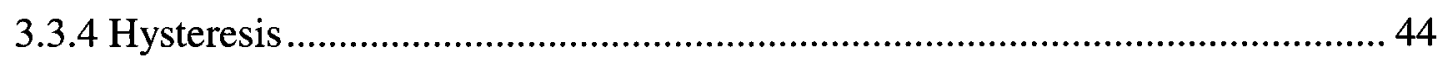

Chapter 4: Elastic Imaging $\quad 46$

4.1 Displacement and Strain Measurement Methods .................................................... 46

4.1.1 Phantom Construction ....................................................................................... 46

4.1.2 Displacement and Strain Imaging................................................................. 48

4.2 Experimental Results and Discussion ............................................................... 59

4.2.1 Displacement and Strain Imaging .................................................................59

4.2.2 Young's Modulus from Ultrasound Strain Images......................................... 73

Chapter 5: Conclusions and Future Work 76

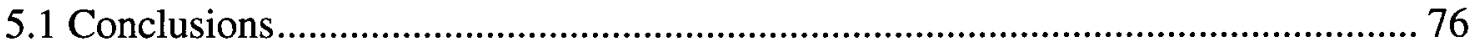

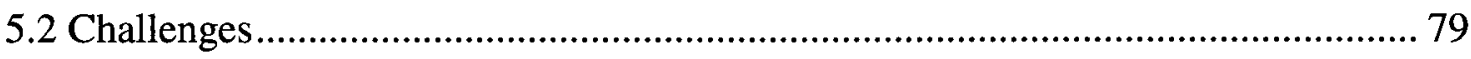

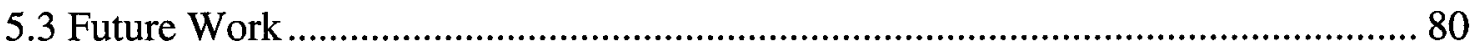

$\begin{array}{lr}\text { Appendix A } & 84\end{array}$

$\begin{array}{lr}\text { References } & 86\end{array}$ 


\section{List of Tables}

Table 1: Results for $0.8 \mathrm{~mm}$ Compression without Multicompression.............70

Table 2: Results for $0.8 \mathrm{~mm}$ Compression with Multicompression................70 


\section{List of Figures}

Figure 2.1:Pulse echo technique.

Figure 2.2:Models of (a) a series of small masses and stiff springs and (b) a series of larger masses and less stiff springs. Copied from [2] 7

Figure 2.3: Models of (a) a series of small masses and weak springs and (b) a series of larger masses and stiffer springs. Copied from [2] .......................................................... 8

Figure 2.4: Diagram to illustrate reflected and transmitted wave....................................... 9

Figure 2.5: Diagrams to illustrate scattering. Copied from [2] ...................................... 10

Figure 3.1: Pictures of (a) silicone mold and (b) device used to slice phantom............... 19 Figure 3.2: Pictures of 3-w\% agar-based phantom constructed and (a) unmolded, (b) slice

Figure 3.3: Schematic diagram of ultrasound echoes used for the velocity measurement of phantom in broadband reflection substitution technique: (a) $\mathrm{V}_{1}$ as a water reference; (b) $V_{2}$ with phantom and $V_{3}$ at water/phantom interface.

Figure 3.4: Block diagram of experimental setup for velocity measurement of phantom. .

Figure 3.5: Picture of ultrasound velocity measurement setup........................................ 23

Figure 3.6: Diagram to illustrate time shifts from echoes. ……………………............... 26

Figure 3.7: Model for Stress-relaxation for soft biological tissue. Copied from [26] ..... 27

Figure 3.8: Block diagram of Stress-relaxation experimental setup................................. 28

Figure 3.9: Setup with (a) top view of probe, mechanical arm, Pyrex container, Plexiglas, balance and phantom and (b) side view of phantom below mechanical arm and probe and above Plexiglas. 29

Figure 3.10: Plot of mechanical arm and probe displacement weight during contact test. 
Figure 3.11: Plot of mechanical arm and probe displacement weight with $0.2 \mathrm{~mm}$

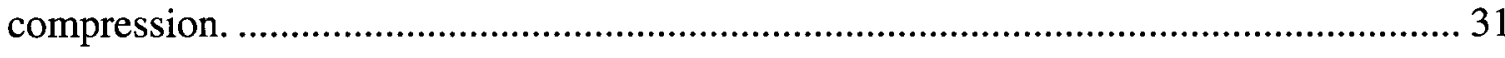

Figure 3.12: Stress-strain curves for (a) elastic and (b) biological materials. ................ 33

Figure 3.13: Picture of hysteresis measurement setup.......................................... 34

Figure 3.14: Ultrasound signals acquired: (a) without phantom, (b) with phantom; and (c) with phantom and foil reflector.......................................................................... 35

Figure 3.15: Ultrasound velocities of phantoms measured with Al foil reflector with

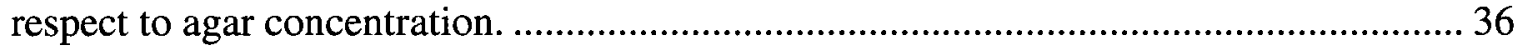

Figure 3.16: Velocity error with respect to $\mathrm{Al}$ foil thickness. ................................. 38

Figure 3.17: Stress-relaxation curves for (a) 2 and (b) 3-w\% agar phantoms................ 39

Figure 3.18: Stress-relaxation curves for $2 \mathrm{~g}$ and $3 \mathrm{~g}$ agar phantoms with (a) $0.2 \mathrm{~mm}$, (b)

$0.4 \mathrm{~mm}$ and (c) $0.8 \mathrm{~mm}$ compression............................................................... 40

Figure 3.19: Stress-relaxation curves for $2 \mathrm{~g}$ and $3 \mathrm{~g}$ agar phantoms with (a) $0.8 \mathrm{~mm}$, (b)

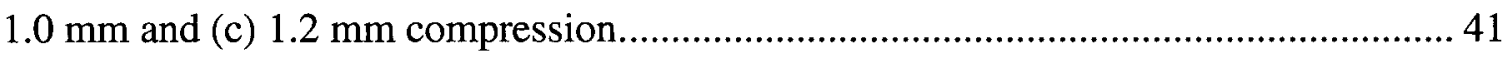

Figure 3.20: Tumor contrast with increasing strain.......................................... 42

Figure 3.21: Stress-strain curves.................................................................... 43

Figure 3.22: Original (markers) and linear fit (solid line) for 2-w\% and 3-w\% agar

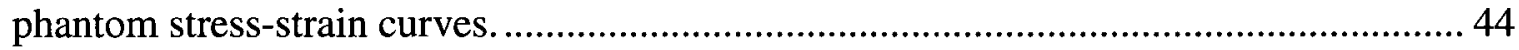

Figure 3.23: Hysteresis for $2 \mathrm{w} \%$ agar phantom................................................ 45

Figure 3.24: Pictures of (a) silicone tumor mold with plastic tube, (b) tumor phantom in uniform mold and (c) unmolded phantom. ........................................................ 47

Figure 3.25: Pictures of (a) device used to slice phantoms for elastic imaging and (b)

sliced composite, tumor phantom. ................................................................ 48

Figure 3.26: Strain profile for (a) uniform springs and (b) unequal springs.................. 49

Figure 3.27: Plot of displacement versus phantom depth...................................... 49

Figure 3.28: Diagram to illustrate scatterer displacement represented with black arrows.

Figure 3.29: Diagrams to illustrate (a) physical system and (b) probe as frame of reference. 
Figure 3.30: Diagram to illustrate RF signals collected for displacement and strain images. Copied from [30].

Figure 3.31: Diagram of pre and post-compression RF signals subdivided into windows. 56

Figure 3.32: Diagram to illustrate overlapping windows to improve axial resolution. .... 57

Figure 3.33: Displacement with poor probe alignment. 59

Figure 3.34: Displacement SNR versus increasing window length. 60

Figure 3.35: Strain images for $0.2 \mathrm{~mm}$ compression (a) before filtering and after median

filtering using a (b) $2 \times 2$ and (c) $3 \times 3$ neighborhood. 62

Figure 3.36: Strain images for $0.2 \mathrm{~mm}$ compression (a) before filter and after median

filtering using a (b) $2 \times 2$ and ( $3 \times 3)$ neighborhood. Colorbar indicates strain. 63

Figure 3.37: Strain image for $0.8 \mathrm{~mm}$ compression (a) before filter and after median filtering using a (b) $2 \times 2$ and (c) $3 \times 3$ neighborhood. 65

Figure 3.38: Strain image for $0.8 \mathrm{~mm}$ compression with multicompression (a) before filtering and after filtering using a (b) $2 \times 2$ and (c) $3 \times 3$ neighborhood............................66

Figure 3.39: Strain image with marked areas used for SNR and contrast calculations.... 69

Figure 3.40: Tumor SNR without and with multicompression....................................... 71

Figure 3.41: Background SNR without and with multicompression................................. 72

Figure 3.42: Contrast before and after median filtering. .................................................. 72

Figure 3.43: Original (markers) and linear fit (solid line) for tumor phantom stress-strain curves. 


\section{List of Symbols}

\begin{tabular}{|c|c|c|}
\hline Symbol & Description & Unit \\
\hline$\overline{A_{m}}$ & Mean signal amplitude & arb \\
\hline$A_{s t d}$ & Standard deviation of the signal amplitude & arb \\
\hline$a$ & Area & $\mathrm{m}^{2}$ \\
\hline B & Bulk modulus & $\mathrm{kPa}$ \\
\hline$c$ & Ultrasound velocity & $\mathrm{m} / \mathrm{s}$ \\
\hline$c_{w}$ & Ultrasound velocity of water & $\mathrm{m} / \mathrm{s}$ \\
\hline$c_{p}$ & Ultrasound velocity of phantom & $\mathrm{m} / \mathrm{s}$ \\
\hline$\Delta c_{p}$ & Velocity error & $\%$ \\
\hline$c_{f}$ & Ultrasound velocity of aluminum foil & $\mathrm{m} / \mathrm{s}$ \\
\hline$C_{\sigma}$ & Contrast from stress measurements & \\
\hline$C_{\varepsilon}$ & Contrast from strain measurements & \\
\hline$d$ & Material depth & $\mathbf{M}$ \\
\hline$d_{i}$ & Local displacement & $\mathrm{Mm}$ \\
\hline$d_{p}$ & Phantom depth & $\mathrm{Mm}$ \\
\hline$d_{f}$ & Aluminum foil thickness & $\mathrm{Mm}$ \\
\hline$d_{w}$ & Window length & $\mathrm{Mm}$ \\
\hline $\mathrm{E}$ & Young's modulus & $\mathrm{kPa}$ \\
\hline$\varepsilon$ & Strain & \\
\hline$f$ & force & $\mathrm{N}$ \\
\hline$f_{c}$ & Center frequency & $\mathrm{MHz}$ \\
\hline$g$ & Gravity & $\mathrm{m} / \mathrm{s}^{2}$ \\
\hline$k$ & Stiffness & $\mathrm{Pa}$ \\
\hline
\end{tabular}




\begin{tabular}{|lll|}
\hline$k_{a}$ & Stiffer spring in series a & $\mathrm{Pa}$ \\
$k_{b}$ & Less stiff spring in series b & $\mathrm{Pa}$ \\
$\lambda$ & Wavelength & $\mathrm{M}$ \\
$m$ & mass & $\mathrm{G}$ \\
$m_{a}$ & Mass of series a & $\mathrm{G}$ \\
$m_{b}$ & Mass of series b & $\mathrm{G}$ \\
$p$ & Pressure & $\mathrm{Pa}$ \\
$p_{r}$ & Reflected pressure & $\mathrm{Pa}$ \\
$p_{i}$ & Incident pressure & $\mathrm{Pa}$ \\
$p_{t}$ & Transmitted pressure & $\mathrm{Pa}$ \\
$\mathrm{R}$ & Amplitude reflection coefficient & \\
$\rho$ & Density & $\mathrm{kg} / \mathrm{m}^{3}$ \\
$\mathrm{SNR}$ & Signal-to-noise ratio & $\mathrm{dB}$ \\
$\sigma$ & Stress & $\mathrm{Pa}$ \\
$\sigma_{\mathrm{t}}$ & Stress of tumor & $\mathrm{kPa}$ \\
$\sigma_{\mathrm{b}}$ & Stress of background & $\mathrm{kPa}$ \\
$\tau_{i}$ & Time shift at each window & $\mathrm{S}$ \\
$\mathrm{t}$ & Time & $\mathrm{S}$ \\
$\Delta t_{l}$ & Time shift between $\mathrm{V}_{1}$ and $\mathrm{V}_{2}$ echoes & $\mathrm{S}$ \\
$\Delta t_{2}$ & Time shift between $\mathrm{V}_{2}$ and $\mathrm{V}_{3}$ echoes & $\mathrm{S}$ \\
$\mathrm{T}$ & Transmission coefficient & $\mathrm{S}$ \\
$T_{i}$ & Time between windows & \\
$v$ & Particle velocity & \\
$\mathrm{V}_{1}$ & Echo from bottom of container (without phantom) \\
$\mathrm{V}_{2}$ & Echo from bottom of container (with phantom0 \\
$\mathrm{V}_{3}$ & Echo from water/phantom interface & \\
\hline & & \\
\hline
\end{tabular}




\begin{tabular}{|lll|}
\hline$z$ & Acoustic Impedance & $\mathrm{Ns} / \mathrm{m}^{3}$ \\
$z_{1}$ & Acoustic Impedance of one material & $\mathrm{Ns} / \mathrm{m}^{3}$ \\
$z_{2}$ & Acoustic Impedance of second material & $\mathrm{Ns} / \mathrm{m}^{3}$ \\
\hline
\end{tabular}




\section{Introduction}

The first chapter introduces this thesis and provides an overview of the entire document and its organization.

\subsection{Overview}

Understanding the mechanical properties of soft tissues, specifically stiffness, is relevant for the diagnosis of many health related illness, such as muscle injury, breast cancer, and liver and heart disease. In addition, providing lower cost and no radiation risk diagnostic tools, such as ultrasound, could make possible safer and better-quality health care for people of all ages. Another motivation behind this research is to gain knowledge about pathological and healthy tissue stiffness and employ this knowledge to differentiate between abnormal and healthy tissue.

\subsection{Problem Statement}

Palpation, examination using touch, is a qualitative method used in medicine to assess tissue stiffness for the detection and diagnose of numerous health-related problems. However, palpation is limited by the examiners experience and the location and size of the tissue changes within the body. Changes in tissue stiffness can indicate pathology (disease) or injury that may require additional diagnostic tests, such as computed tomography (CT), magnetic resonance imaging (MRI), traditional ultrasound and contrast-enhanced ultrasound (CEUS). However, CTs emit radiation, MRIs are 
expensive and involve waiting lists. Since traditional medical ultrasound and CEUS measures tissue echogenicity (echoes from reflection or scattering) tumors may go undetected. Ultrasound elastography could provide a no radiation risk, low cost diagnostic method for all of society. In addition, elastography research could increase knowledge of healthy tissue stiffness.

\subsection{Objectives}

The objectives of this research were to construct and characterize soft tissue mimicking phantoms and demonstrate ultrasound elastography using the phantoms developed. The first objective was to characterize one acoustic (ultrasound velocity) and three mechanical properties (stress-relaxation, hysteresis and Young's modulus) of single concentration phantoms, constructed to resemble biological soft tissue. The results of the first objective were important to facilitate the second and primary objective, ultrasound elastography research using a composite, which resembled a tumor, of the single concentrations phantoms.

\subsection{Thesis Contributions}

The following is a list of contributions as explained in this thesis:

\section{Research Contributions:}

- Developed and implemented a novel signal processing technique to improve the quality (SNR) of strain images. Multicompression in elastography literature is a common experimental method that replaces a larger physical compression with smaller step-wise physical compressions to improve displacement and strain 
images. Multicompression in this thesis replaces the physical experimental method with a signal processing method.

- Developed and implemented a novel technique to measure the ultrasound velocity of phantoms and a model that assessed the velocity error associated with implementing the technique. This achievement has been presented as follows. $\mathbf{R}$. Morehouse and Y. Ono, "An Ultrasound Velocity Measurement Technique for

Tissue-Mimicking Phantoms for Ultrasound Imaging", Proceedings of $33^{\text {rd }}$ Canadian Medical and Biological Engineering Conference, June 2010, Vancouver, BC.

Technical Contributions:

- Implemented experimental procedures to produce phantom stress-relaxation and stress-strain curves and to collect ultrasound data for phantom displacement and strain images.

- Implemented existing correlation and spline interpolation techniques to produce and improve displacement and strain images.

- Developed and implemented algorithms to pre-process raw data from ultrasound imaging system.

\subsection{Thesis Organization}

Chapter 1 introduces the subjects discussed throughout this thesis.

Chapter 2 provides background information about the principles of ultrasound and the methods of determining tissue stiffness (palpation and elastic imaging), along with two 
mechanical properties of biological soft tissues (stress-relaxation and hysteresis) and one acoustic property (ultrasound velocity).

Chapter 3 describes phantom development and construction of single concentration phantoms, along with characterization experimental methods (ultrasound velocity, stressrelaxation, hysteresis, and Young's modulus), results and discussion.

Chapter 4 describes tumor phantom development and construction, along with displacement and elastic imaging experimental methods, results and discussion. Young's modulus is also determined from ultrasound strain images.

Chapter 5 concludes this thesis, describes the research challenges, and then discusses recommended future work. 


\section{Chapter 2: \\ Background Review}

Ultrasound elastic, or strain imaging, a non-invasive method of measuring the elastic properties of soft tissues, is investigated. In this thesis various concentrations of agar-based phantoms were developed and characterized, specifically ultrasound velocity, stress-relaxation and hysteresis were determined. Ultrasound displacement and strain images were created using various agar-based concentrations and composite phantoms, and then Young's modulus was determined for two different phantom concentrations.

This chapter explains the principles of medical ultrasound imaging, the common methods used to determine soft-tissue stiffness and the background of phantom characterization relevant to this thesis.

\subsection{Principles of Ultrasound}

\subsubsection{Ultrasound Physics}

Sound waves are mechanical pressure waves that propagate through materials by expanding and compressing particles in the material [1]. In an ultrasound imaging system the particles move forward and back in the same direction as the acoustic wave propagation, thus the wave is called a longitudinal wave [1].

\subsubsection{Ultrasound Velocity}

Ultrasound velocity of a material, $\mathrm{c}$, can be determined by: 


$$
c=\frac{2 d}{t}
$$

where $d$ is the depth of the material and $t$ is the time it takes for the sound wave, or echo, to propagate through the material and return using a pulse echo technique. The depth is multiplied by 2 for a round trip scenario, as shown in Fig. 2.1.

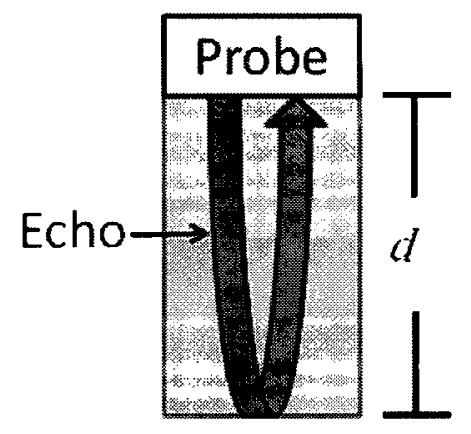

Figure 2.1:Pulse echo technique.

The Ultrasound velocity of a material or phantom depends on the materials density, $\rho$, with units $\mathrm{kg} / \mathrm{m}^{3}$ and coefficient of stiffness, $k$, with units of $\mathrm{Pa}$ [2], [3].

$$
c=\sqrt{\frac{k}{\rho}}
$$

To demonstrate how density and stiffness determine ultrasound velocity Fig. 2.2 shows two series of masses connected by springs [2]. The series in Fig. 2.2 (a) has small masses, $m_{a}$, and the series in Fig. 2.2 (b) has larger masses, $m_{b}$, representing low and high density, respectively [2]. The small masses are connected by stiffer springs, $k_{a}$, and the larger masses are connected by less stiff springs, $k_{b}$ [2]. A longitudinal wave pushing on the first mass of each series would propagate to the next mass via the springs [2]. In Fig. 2.2 (a) the masses are light and the springs are stiffer, so the wave would travel quickly, but in Fig. 2.2 (b) the masses are heavier and the springs are less stiff, so the wave would 
travel slower [2]. Thus, ultrasound velocity depends on the material it is moving through, and varies through different types of tissue. However, the average ultrasound velocity for biological soft tissue is $1540 \mathrm{~m} / \mathrm{s}$ and most soft tissue lies within $5 \%$ of the average [2]. For example, the ultrasound velocities of fat and muscle are $1470 \mathrm{~m} / \mathrm{s}$ and $1600 \mathrm{~m} / \mathrm{s}$, respectively [4].

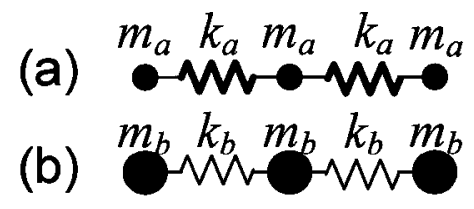

Figure 2.2:Models of (a) a series of small masses and stiff springs and (b) a series of larger masses and less stiff springs. Copied from [2].

\subsubsection{Acoustic Impedance and Reflection}

How particles respond in a material to a wave of pressure (longitudinal wave) is dependent on the acoustic impedance of that material, $\mathrm{z},[2]$.

$$
z=\frac{p}{v}
$$

where $p$ and $v$ are the local pressure and particle velocity, respectively. The acoustic impedance of a material, $z$, depends on the density and stiffness of the material [2]:

$$
z=\sqrt{\rho k}
$$

Acoustic velocity can also be defined in terms of velocity [2]:

$$
z=\rho c
$$


Acoustic impedance can also be modeled with a series of masses and springs [2]. In Fig. 2.3 (a) the masses, $m_{a}$, are light and the springs, $k_{a}$, are weak [2]. The first light mass is easily accelerated by a wave and receives little resistance from the adjacent weak spring, thus mass movement is easy and the overall impedance in the series is low [2]. In Fig. 2.3 (b) the heavy masses, $m_{b}$, are harder to accelerate with the same wave and movement is further reduced by the stiff spring, $k_{b}$, and thus the impedance in the series is high [2].

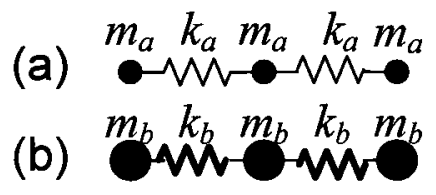

Figure 2.3: Models of (a) a series of small masses and weak springs and (b) a series of larger masses and stiffer springs. Copied from [2]

A sudden change in acoustic impedance from a change in the ratio of pressure to particle velocity at boundaries, such as organ interfaces, generates a reflected wave [2]. Part of the incident wave is reflected, thus produces an echo in a round trip scenario, and the other part is transmitted, as shown in Fig. 2.4. The amplitude of the reflected wave, or amplitude reflection coefficient, $R$, is [1]:

$$
R=\frac{p_{r}}{p_{i}}=\frac{z_{2}-z_{1}}{z_{2}+z_{1}}
$$

where $p_{r}$ and $p_{i}$ are the pressure amplitudes of the reflected and incident wave, respectively, and $z_{1}$ and $z_{2}$ are the acoustic impedances of the different materials, as shown in Fig 2.4. Since the entire incident pressure is reflected or transmitted: 


$$
p_{i}=p_{\mathrm{t}}-p_{r}
$$

where $p_{t}$ is the pressure amplitude of the transmitted wave, therefore the amplitude transmission coefficient is [1]:

$$
T=R+1
$$

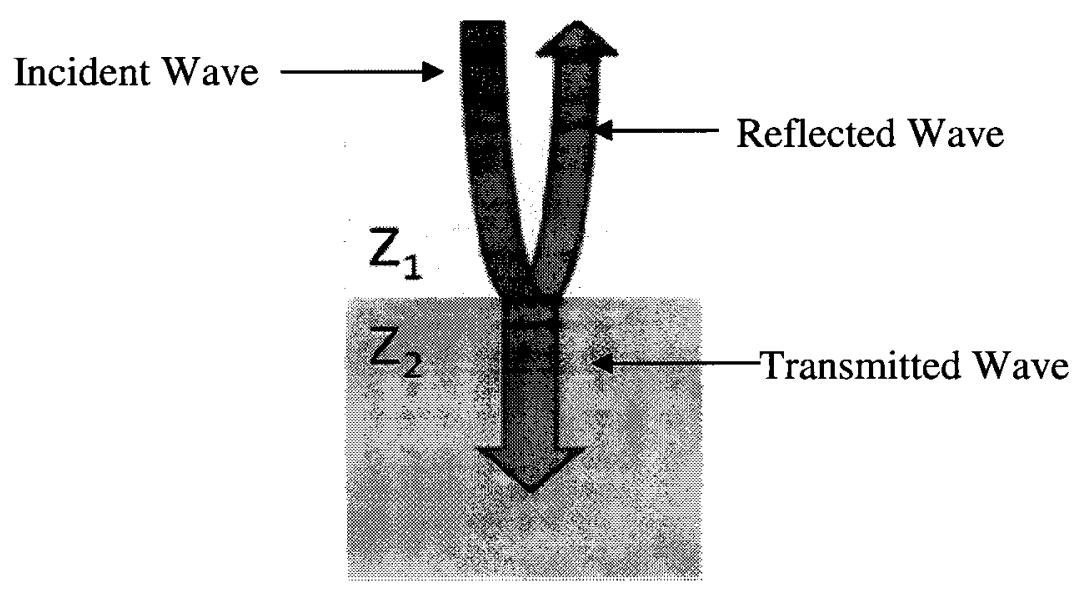

Figure 2.4: Diagram to illustrate reflected and transmitted wave.

\subsubsection{Scattering}

When an acoustic wave encounters a small or rough particle within a material, the incident wave is scattered in many directions, as shown in Fig. 2.5 [2]. A small particle would be considered smaller than the wavelength, $\lambda$, of the incident wave, as shown in Fig. 2.5. Backscatter is the returned acoustic wave from scattering of the incident wave. Reflection provides images of tissue boundaries, such as organ and blood vessel boundaries, while backscatter provides images of boundaries and parenchyma (nonsupporting tissues within an organ) [5]. Scattering material, such as graphite particles, is added to phantoms to mimic tissue parenchyma, thus elastographic imaging can be performed. 


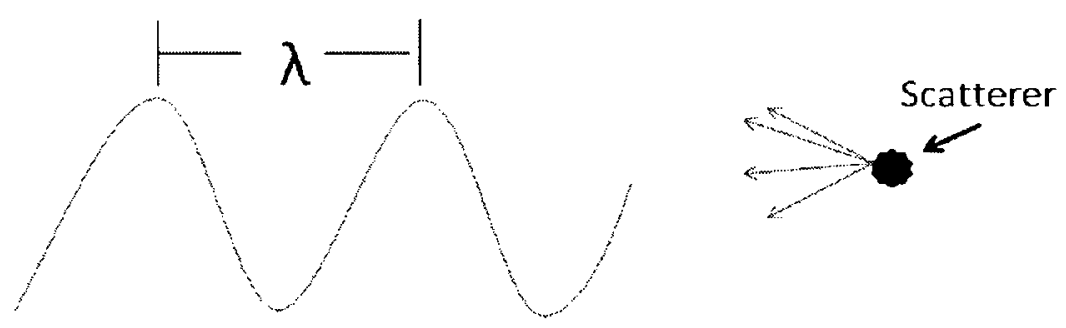

Figure 2.5: Diagrams to illustrate scattering. Copied from [2].

\subsection{Methods of Determining Tissue Stiffness}

Biological soft tissues are complex in structure, responses and anisotropic (mechanical properties that vary with angle) [6]. Soft tissues can be classified as homogeneous (uniform distribution), inhomogeneous (small fluctuations in distribution) or heterogeneous (large variations in spatial distribution). Soft tissues are also inelastic and some soft tissues are either isotropic, such as fat, while others, such as muscle, are anisotropic. To simplify the complex nature of tissues, they are often assumed to be homogeneous, linearly elastic and isotropic. Investigating and understanding the viscoelastic properties of tissues, such as stress-relaxation, hysteresis, and creep, helps to provide insight into the factors that affect tissue elasticity and elastography, or strain imaging. In addition, understanding and investigating the acoustic properties of these complex tissues, such as ultrasound velocity, scattering and attenuation, is also beneficial to understanding wave propagation in tissue [6], [7]. Since soft tissue phantoms are constructed to resemble the mechanical and acoustic properties of soft tissues, phantom research is also important so in-vitro conditions represent clinical results [7], [8]. 


\subsubsection{Palpation and Elastic Imaging}

Ultrasound elastography is a quantitative method for imaging the elasticity, or compressibility, of biological soft tissues; however the role of palpation in medicine remains the standard for determining tissue stiffness or hardness [9]. Palpation is used to access tissue injury, fractures and detect tumors. Detecting tumors with palpation is limited by the examiner's experience, and the tumor's location and size within the body [10]. Tissue contrast, measured by traditional sonography, is mechanically unrelated to tissue stiffness [10], thus an abnormal, stiff tumor may go undetected by traditional ultrasound, or sonography. The goal of elastography is not to replace sonography, but to emulate palpation, thus provide tissue stiffness data.

Elastography plays an important clinical role, since it has been used to successfully image breast, thyroid, prostate gland, and lymph node tumors [6], [11]. Other applications include monitoring liver disease progression, myocardial intravascular ultrasound imaging (IVUS), vein thrombosis detection and muscle or tendon injury [11]. Elastography research has increased knowledge of tissue structure along with normal tissue stiffness differences [10]. Most research methods in elastography currently report relative tissue stiffness data, nevertheless these measurements have also shown to be beneficial [4].

The measurements that most closely approximate tissue palpation or stiffness are Young's modulus and shear modulus. Young's modulus is the ratio of longitudinal stress to longitudinal strain in a material, while shear modulus is the ratio of transverse stress to transverse strain in a material [4]. Elastography is a method which generates 
elastograms, or strain images [10]. Sonoelastography is elastography using ultrasound. If the applied stress is known or measured and the resultant strains are known or measured, then Young's modulus of a material can also be determined.

Elastography cannot be measured directly, so all elastographic methods involve indirectly measuring strain by applying an external or internal stimulus and measuring tissue response, or motion. There are three general methods to image tissue elasticity with ultrasound [6]. The first method is called quasi-static, which as the name implies, involves applying a small, almost static compression to materials and determining the resultant tissue displacement or strain. The term 'static' is used because the data acquisition time is much less compared to loading time [12]. The second method is a dynamic method called sonoelaticity and involves using low-frequency vibrations applied to tissue from a vibrating source [12]. The resultant displaced waves are detected using Doppler [12]. The third method is called organic because natural movements of the body, such as vascular contractions, create the stimulus [6].

Magnetic resonance elastography (MRE) is a combined method that modulates transverse ultrasonic waves using the magnetic field gradient of a magnetic resonance imaging (MRI) scanner [6]. The MRI images propagating transverse waves and shear modulus is calculated using prior knowledge of wavelength and tissue density [13].

To implement the quasi-static method, which was used for all elastographic images in this thesis, an ultrasound scanner that collects radio-frequency $(\mathrm{RF})$ ultrasound signal, a phantom or tissue sample and signal analysis software, such as Matlab are needed. Digital filtering techniques may also prove worthwhile. The ultrasound probe can 
be pressed onto a phantom and the RF signal can be acquired [10]. After compressing the phantom more a second RF signal can be acquired [10]. The displacement of the phantom creates a time shift in the ultrasound echoes and the time lag of the echo peaks can be determined using a cross-correlation technique. Since the time shift is proportional to displacement, and strain is the gradient of displacement, displacement and strain can be obtained from the time shifts [10].

Other post-signal processing methods used to estimate time shifts include frequency and phase shift analysis [14]. The primary disadvantages to frequency and phase shift analysis is the limitation and thus uncertainty of measurements when axial motion is greater than a quarter of an ultrasonic wavelength [15]. This problem is not seen with correlation methods, which is performed in the time domain. One disadvantage of correlation is decorrelation, which appear as displacement or strain amplitude spikes in images [16]. Another issue with correlation is that the ultrasound system sampling rate may not be sufficient to detect time shifts that are used to calculate strains [15]. Various methods used to improve time shift accuracy, such as Hilbert transforms and interpolation, and signal to noise ratio, such as filtering are investigated and discussed [16].

\subsection{Tissue Mimicking Phantom Characterization}

\subsubsection{Ultrasound Velocity}

Ultrasound phantoms are used in ultrasound biomedical research to calibrate medical and research ultrasound equipment for quality control and performance testing, 
and to train technicians [7], [17]. They are fabricated to resemble the acoustic properties of soft biological tissues, thus it is important to accurately evaluate their ultrasound velocity, attenuation and scattering characteristics [7], [8]. The International Electrotechnical Commission 1390 (IEC) and the American Institute of Ultrasound in Medicine Standard 1990 suggests that ultrasound phantoms have a velocity of $1540 \mathrm{~m} / \mathrm{s}$ [7], [8]. Alteration in ultrasound velocity for calibration phantoms away from $1540 \mathrm{~m} / \mathrm{s}$ leads to blurred images [17] from beam focusing and inaccurate distance measurements [18], and thus impacts medical diagnosis. Therefore, it is important to evaluate the ultrasound velocity accurately in the phantom development and/or fabrication.

Ultrasound velocity, $c$, of a sample can be obtained by: $c=2 d / t$ as shown in eqn. 2.1. However, it is often difficult to measure the thickness of a soft tissue phantom with the desired accuracy using a caliper or micrometer, since the physical contact of such devices with the sample may deform and/or damage the phantom, resulting in deterioration of velocity measurement accuracy. In an effort to improve the measurement accuracy, various approaches have been explored [19].

A broadband reflection substitution technique is one of the methods used to measure the ultrasound velocity of materials [20]. In this method, the time delay difference of ultrasound echoes reflected from the top and bottom surfaces of the sample immersed into water is measured. Then, the ultrasound velocity of the sample is calculated using that of water as a reference. Therefore, the direct measurement of the sample thickness is not required. However, it is often difficult to observe an echo, having 
a good signal-to-noise ratio (SNR), reflected at the water/phantom interface, because the acoustic impedances of soft-tissue phantoms are close to that of water.

In order to improve the SNR of the echo reflected at water/phantom interface, a technique to place a reflector between the water and the phantom has been proposed and investigated. An aluminum (Al) foil reflector having a thickness of $15 \mu \mathrm{m}$ was placed on the top surface of the phantom. Using the proposed technique, the ultrasound velocities of five agar-based phantoms with varying agar concentrations were measured at room temperature. In addition, the effect of the $\mathrm{Al}$ foil thickness on the velocity measurement accuracy was investigated. 


\section{Chapter 3: \\ Tissue Mimicking Phantom Construction and Characterization}

This chapter explains the construction and characterization of tissue mimicking materials, or phantoms. Ultrasound phantoms, which represent the acoustic and mechanical properties of biological soft tissue, are constructed as they are needed for invitro elastography research. First, the materials chosen to construct all the phantoms used in this thesis, specifically characterization and elastic imaging are presented. Next, the phantom construction and characterization methods, specifically ultrasound velocity, stress-relaxation, Young's modulus and hysteresis are explained. To conclude this chapter, characterization results are presented and discussed.

\subsection{Tissue Mimicking Phantom Construction}

It was decided to use agar and graphite powder as materials for constructing phantoms for all experiments conducted in this thesis. Agar is made from seaweed and was laboratory grade. Since agar and graphite phantoms were developed for elastic imaging it was decided to also characterize the agar and graphite phantoms. Agar and agar/gelatine combinations have been commonly used in the past to mimic the acoustic and elastic properties of soft biological tissue [21], [22], [23]. Agar has also been used successfully to mimic organs, breast tissues, sinus cavities and vascular systems [22]. 
Graphite powder was added to each phantom for absorption and ultrasound scatterers for imaging. The amount of graphite added to each phantom to achieve uniform distribution and sufficient scatters for imaging was investigated previously [23], so the same amount of graphite (3-w\%) with the same particle size composition of $6.813 \mu \mathrm{m}(10 \%), 26.03$ $\mu \mathrm{m}(50 \%)$ and $50.33 \mu \mathrm{m}(90 \%)$ in diameter was used in this thesis. The graphite powder was purchased from Alfa Aesar (Ward Hill, MA, USA).

Although agar was used for this thesis, alginate was also considered. It is clear that each material has advantages and disadvantages. The advantages of alginate were that it set very quickly (within $10 \mathrm{~min}$ ) and the shelf-life of the material was longer than agar. The disadvantage of using alginate was that it required blending with a hand-held mixer, which introduced air and thus required additional degassing. The alginate phantoms were also more elastic (rubber-like) to touch and thus more difficult to slice. The advantages to agar were the shelf-life, once the agar phantom were stored in distilled water they remained usable for months, and they sliced cleanly. Using different concentrations of agar allowed phantoms to have varying stiffness and an ultrasound velocity close to that of biological tissue $1540 \mathrm{~m} / \mathrm{s}$ [2]. The disadvantages of agar phantoms were the slow set time and the fragility of the phantoms at low concentrations.

The following procedure was used to construct five homogeneous agar-based phantoms with agar concentrations of 1, 1.5, 2, 2.5 and 3-w\% for the investigation of the effect of agar concentration on phantom ultrasound velocity. An outline and flowchart of the construction method for a uniform phantom is located in Appendix A. Distilled water was boiled using a metal kettle and hot plate for 30 minutes to degas, and then $100 \mathrm{ml}$ of 
degassed water was slowly poured into a titled glass beaker to prevent introducing air into the water. Agar was slowly added (for instance, $1 \mathrm{~g}$ for $1-\mathrm{w} \%$ phantom), which prevented clumping, while the water was stirred, which facilitated combining the agar and water. The mixture was heated, using a hot water bath placed on a hot plate, to above $80{ }^{\circ} \mathrm{C}$ to dissolve the agar, (melting point of agar $85{ }^{\circ} \mathrm{C}$ ) and then stirred to combine. Heating and stirring was repeated twice until the mixture appeared to have changed from cloudy to clear. Because of evaporation, water was added to maintain the mixture at $100 \mathrm{ml}$. The mixture was then stirred until the temperature measured $35^{\circ} \mathrm{C}$. At this temperature the mixture became more viscous, and thus was capable of suspending particles. If the mixture was allowed to cool below $35^{\circ} \mathrm{C}$ then a film formed on the surface and the mixture started to set, thus more components could not be added without clumping and the mixture could not be poured.

Graphite powder was added as ultrasound scatterers while the mixture was stirred more vigorously. If the stir speed was too slow the powder would sit on the surface of the mixture and not become combined, thus an adequate stir speed (vortex on the top observed but not deep enough to reach the stir stick, which would add air to the mixture) allowed the mixture and graphite powder to combine. Graphite powder has very little effect on velocity [8], [24]. It is the graphite that gives the phantoms their black color.

The phantom was poured into a silicone rubber mold, shown in Fig. 3.1 (a), and then place into an ice water bath for a minimum of one hour to quickly set the agar and prevent gradients in the concentration of the graphite particles. The phantom was then 
unmolded, wrapped in plastic wrap, labeled (phantom number, date and amount of components) and stored in the fridge.

Phantoms were sliced using a device composed of two blades separated in parallel by $23 \mathrm{~mm}$ spacers, as shown in Fig. 3.1 (b). Fig. 3.2 (a) presents a picture of an unmolded 3-w\% agar phantom. The unmolded phantom was sliced along the dotted lines, as indicated in Fig. 3.2 (a), to make a phantom sample (dimensions approximately $23 \mathrm{x} 40 \mathrm{x}$ $50 \mathrm{~mm}$, as shown in Fig. 3.2 (b), used in the velocity measurement experiment.
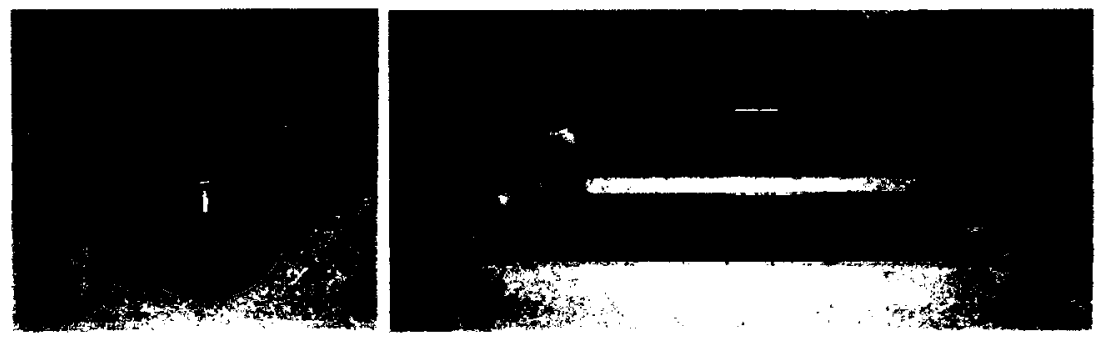

Figure 3.1: Pictures of (a) silicone mold and (b) device used to slice phantom.

(a)

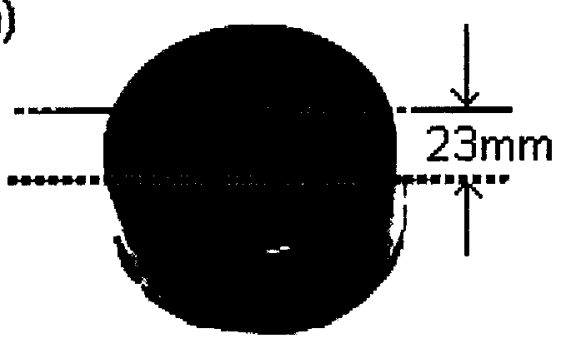

>

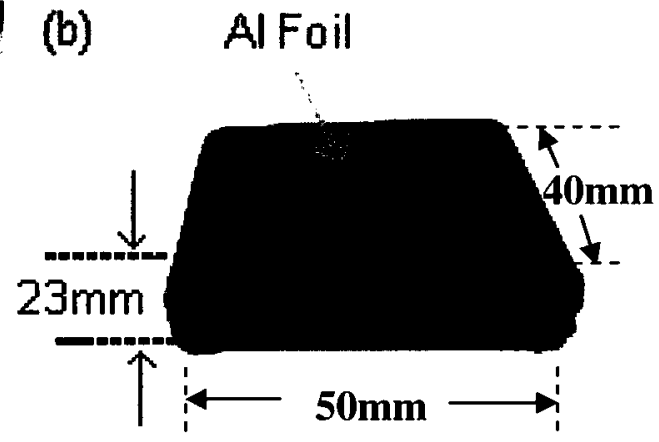

Figure 3.2: Pictures of 3-w\% agar-based phantom constructed and (a) unmolded, (b) slice with aluminum $(\mathrm{Al})$ foil reflector on surface.

Isotropic, single concentration phantoms with 2 and $3-w \%$ agar were also constructed for visco-elastic experiments (stress-relaxation and hysteresis) and to determine Young`s modulus, discussed later in this chapter, and for elastic imaging, 
discussed in Chapter 5. Since the visco-elastic experiments, compared to ultrasound velocity experiments, were performed later in time the procedure to construct phantoms changed slightly in order to improve the reproducibility of the phantom construction; these changes are discussed below.

The temperature of the degassed water was measured and ensured to be above 90 ${ }^{\circ} \mathrm{C}$ before pouring into a beaker. In addition, the beaker was also heated, by immersing in boiling water, before receiving the water. Ensuring the water and the beaker were heated allowed the agar to combine easier and reduced the time required to reheat the mixture. The mixture was also covered when heated and stirred, thus preventing evaporation and the need to add water. Also, a hotpot instead of a pot on a hotplate was now used to heat the hot water bath that was used to heat the mixture. Using a hotpot was more stable, and thus safer. To reduce the time needed to construct the phantoms the mixture was cooled to $50^{\circ} \mathrm{C}$ before graphite powder was added. It was determined that using the ice water bath and reducing the temperature to $50{ }^{\circ} \mathrm{C}$, instead of $35{ }^{\circ} \mathrm{C}$, was sufficient to prevent gradients in the concentration of graphite particles. A higher temperature was not investigated.

The molded phantoms were also placed in the ice water bath overnight instead of being placed in the fridge. In the morning the phantoms were at room temperature, so the phantoms did not need to reach room temperature before experiments could start. It was also observed that the stiffness and shape of the phantom changed when the temperature of the phantom changed. Since all experiments were done at room temperature, the phantoms were sliced at room temperature. 


\subsection{Tissue Mimicking Phantom Characterization Methods}

\subsubsection{Ultrasound Velocity}

In order to improve the SNR of the echo reflected at water/phantom interface, a technique to place a reflector between the water and the phantom has been proposed and investigated. An aluminum (Al) foil reflector having a thickness of $15 \mu \mathrm{m}$ was placed on the top surface of the phantom. Using the proposed technique, the ultrasound velocities of five agar-based phantoms with varying agar concentrations were measured at room temperature. The effect of the $\mathrm{Al}$ foil thickness on the velocity measurement accuracy will be discussed in section 3.3.1.

Fig. 3.3 shows the ultrasound echoes used for the velocity measurement of a phantom in the broadband reflection substitution technique. $V_{1}$ and $V_{2}$ are the echoes reflected from the bottom of a water container with and without a phantom, respectively, and $V_{3}$ is the echo from the water/phantom interface. Using the literature value of the velocity of a reference medium, such as distilled water $\left(c_{w}\right)$ [25], at a known temperature, the ultrasound velocity of a phantom $\left(c_{p}\right)$ can be calculated using the following equation:

$$
\frac{1}{c_{p}}=\frac{1}{c_{w}}+\frac{\Delta t_{1}}{2 d_{p}}
$$

where $\Delta t_{1}$ is the time shift between the $\mathrm{V}_{1}$ and $\mathrm{V}_{2}$ echoes and $d_{p}$ is the thickness of the phantom. Since $2 d_{p}=\Delta t_{2} \cdot c_{p}$, where $\Delta t_{2}$ is the time shift between the $\mathrm{V}_{2}$ and $\mathrm{V}_{3}$ echoes, $c_{p}$ is given by:

$$
c_{p}=c_{w}\left(1-\frac{\Delta t_{1}}{\Delta t_{2}}\right)
$$


Using eqn. 3.2, $c_{p}$ is determined with signal analysis of three echoes, $\mathrm{V}_{1}, \mathrm{~V}_{2}$ and $\mathrm{V}_{3}$, as shown in Fig. 3.3, thus the direct measurement of the phantom thickness is not required.

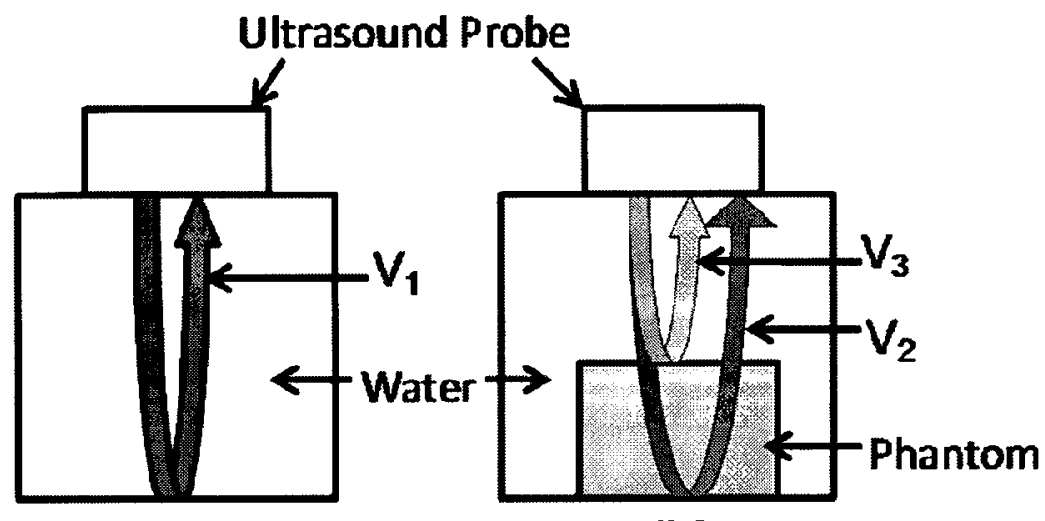

(a)

(b)

\section{Reference Signal Phantom Signal}

Figure 3.3: Schematic diagram of ultrasound echoes used for the velocity measurement of phantom in broadband reflection substitution technique: (a) $\mathrm{V}_{1}$ as a water reference; (b) $\mathrm{V}_{2}$ with phantom and $\mathrm{V}_{3}$ at water/phantom interface.

Fig. 3.4 presents a block diagram of the experimental setup used to measure the ultrasound velocity of the phantoms and Fig. 3.5 shows a picture of the ultrasound velocity experimental setup. A Pyrex glass container was filled with distilled water. A flat plate of Plexiglas with a thickness of $12.7 \mathrm{~mm}$ was fixed onto the bottom of the container to ensure clear echoes of $V_{1}$ and $V_{2}$. A thermocouple was inserted into the water to measure the water temperature with a resolution of $0.1{ }^{\circ} \mathrm{C}$. The thermocouple was also used to measure the temperature of the phantom. 


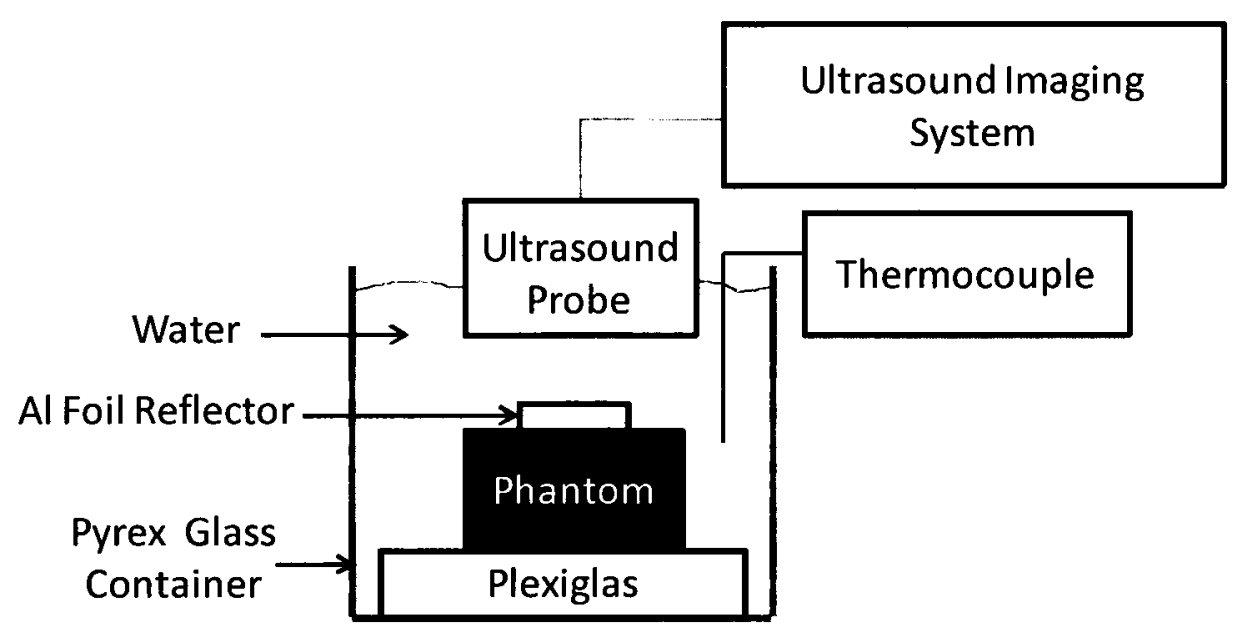

Figure 3.4: Block diagram of experimental setup for velocity measurement of phantom.

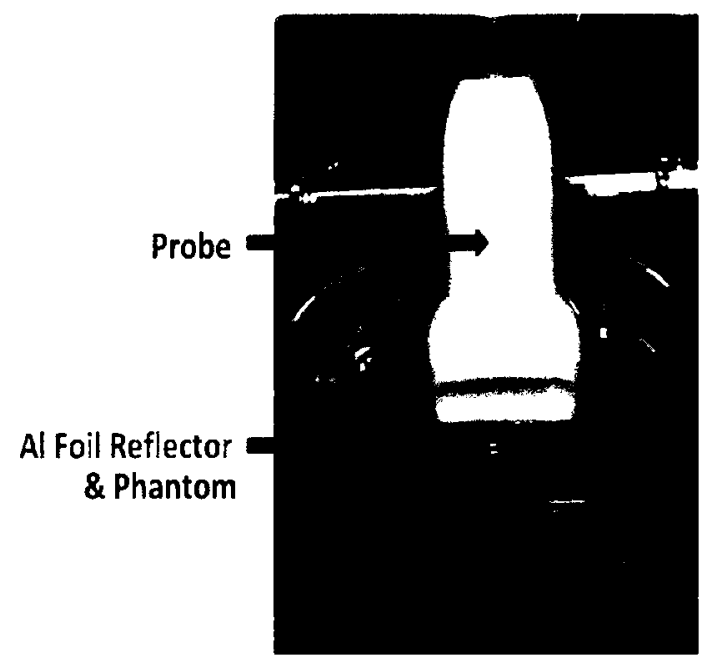

Figure 3.5: Picture of ultrasound velocity measurement setup.

A linear array ultrasound probe (L10-5, ESAOTE, Maastricht, The Netherlands) was connected to a medical ultrasound imaging system (Picus, ESAOTE, Maastricht, The Netherlands) and the probing surface was immersed into water at a depth that allowed 
enough room for the phantom to be placed underneath the transducer. The ultrasound center frequency, $\mathrm{f}_{\mathrm{c}}$, was $5 \mathrm{MHz}$. Alignment of the ultrasound beam normal to the Plexiglas plate was accomplished by adjusting the angle of the probe so as to obtain the maximum amplitude of the $V_{1}$ echo reflected from the Plexiglas plate. An aluminum foil reflector with area of $5 \mathrm{~mm}$ by $5 \mathrm{~mm}$ was placed on the top of the phantom sample. The foil thickness, $d_{f}$, was $15 \mu \mathrm{m}$ measured using a micrometer.

First, the temperature of the water was measured with a thermocouple, and then the water reference $V_{1}$ echo without the phantom was acquired. Before immersing the phantom sample into water, a droplet of water was placed on the foil and then the wet foil reflector was placed on the top of the phantom to ensure that no air bubbles were trapped between the phantom and the foil. The phantom with the foil was immersed into water and placed on the Plexiglas plate. The temperature of the phantom was measured using a thermocouple and the temperature difference between the water and the phantom was verified to be within $0.1{ }^{\circ} \mathrm{C}$. Then, the $V_{2}$ and $V_{3}$ echoes were acquired. The ultrasound signals were acquired with a sampling frequency of $33 \mathrm{MHz}$ and an $\mathrm{A} / \mathrm{D}$ resolution of 12 bits.

Acquired ultrasound signals were analyzed using Matlab. Before the RF signals were processed the header information and the lens signals, the first 16 and 69 data points, respectively, were removed from the RF signals.

The time shifts of $\Delta t_{1}$ between $V_{1}$ and $V_{2}$ and $\Delta t_{2}$ between $V_{2}$ and $V_{3}$, as shown in Fig. 3.6, were determined using signal processing techniques. Correlation is a common method used to estimate signal time shifts, or delays [15], [19]. Correlation determines 
signal similarities either using one signal (auto-correlation), which determines how similar waveforms are at different times, or using two signals (cross-correlation) which determines the similarity between two waveforms. To determine time shifts, Matlab's built in function "xcorr" was used to generate correlated signals. The correlated signals amplitudes are correlation coefficients, which indicate the degree of similarity. A correlation coefficient of 1 (normalized from 0 to1) occurs if two signal waveforms are identical.

A Hilbert transform on the correlated signals generated analytic signals, and then computing the magnitudes of the analytic signals gave the envelopes [19]. The envelopes of the signals removed possible phase changes that could occur at the phantom interfaces.

In addition, spline interpolation at the peak of the envelope was performed to improve the time shift measurement accuracy [16], because the peak determined in discrete data was not always the best estimate of the actual peak location. Improving the accuracy of the peak location improved time shift accuracies, and thus velocity measurement accuracy.

Spline interpolation was performed using Matlab's built in function "spline," which applies a cubic (third order) spline to connect data points. Cubic splines are preferred for this research as they have a smoothing affect at the peak data point (slope does not change rapidly as with linear splines). To improve processing time the entire length of the cross-correlation signal was not splined, but rather the data points before and after the peak data point were selected as end points (series of three data points), and 200 intervals were used between end points. 
To determine $\Delta \mathrm{t}_{1}$, the time zero index (for zero lag) from the auto-correlation of the reference signal was subtracted from the shifted time index (splined peak of the envelope) of the cross-correlation of the reference and phantom signals, and then multiplied by the sampling interval. To determine $\Delta \mathrm{t}_{2}$, the indices (splined peaks of the envelope) of the auto-correlation of the phantom signal were subtracted, and then multiplied by the sampling interval.

The ultrasound velocity of water, $c_{w}$, was obtained using literature [25] and the water temperature measured during the experiment. Then, the ultrasound velocity, $c_{p}$, of the phantom was calculated using eqn. 3.2.

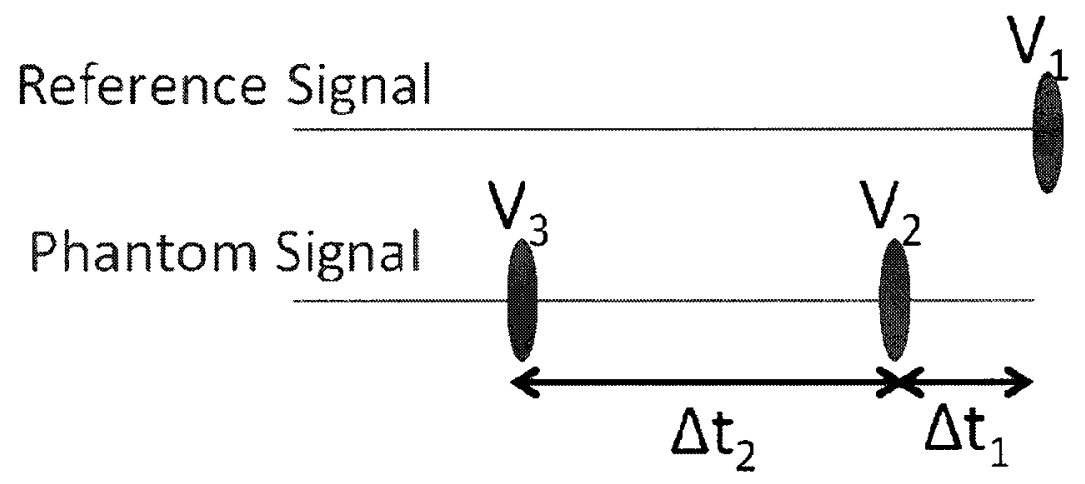

Figure 3.6: Diagram to illustrate time shifts from echoes.

\subsubsection{Stress-Relaxation}

When biological tissues are strained and the strain is kept constant over time, as shown in Fig. 3.7, the stress, $\sigma$, in the tissue does not remain constant, but decreases in time [26]. This behaviour, or phenomenon, is called stress-relaxation. 


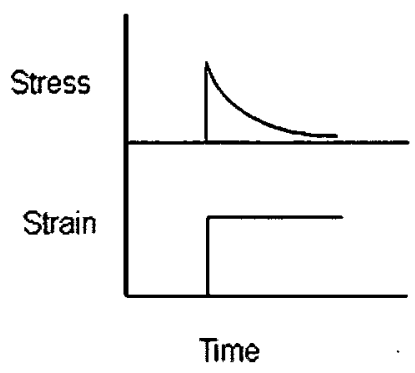

Figure 3.7: Model for Stress-relaxation for soft biological tissue. Copied from [26].

Fig. 3.8 shows a block diagram of the experimental setup used to acquire stressrelaxation measurements. A linear array probe (L10-5, ESAOTE, Maastricht, The Netherlands) was connected to an ultrasound imaging system (Picus, ESAOTE, Maastricht, The Netherlands). The probing end of the linear array probe was inserted into the opening of the mechanical arm, flushed with the bottom surface of the mechanical arm, and then secured with adhesive tape and wire. The probe was not required in stressrelaxation measurements, but the same experimental setup was also used for displacement and strain imaging. A Pyrex container with a glued piece of Plexiglas on the bottom was placed on a balance (EJ-4100, A\&D Company, Tokyo, Japan, resolution $0.1 \mathrm{~g})$. The balance, Plexiglas and mechanical arm were then levelled. Distilled water was poured into the bowl to a depth above $30 \mathrm{~mm}$. The experiment was performed in water to prevent phantom dehydration and to provide slip free boundary conditions [27]. The exact depth of the water was not important, except to completely cover the phantom during measurements. The $2-\mathrm{w} \%$ agar phantom was sliced $30 \mathrm{~mm}$ cubic, and then placed 
on the Plexiglas, directly under the probe surface and completely submerged. Pictures of the setup are shown in Fig. 3.9 (a) and (b).

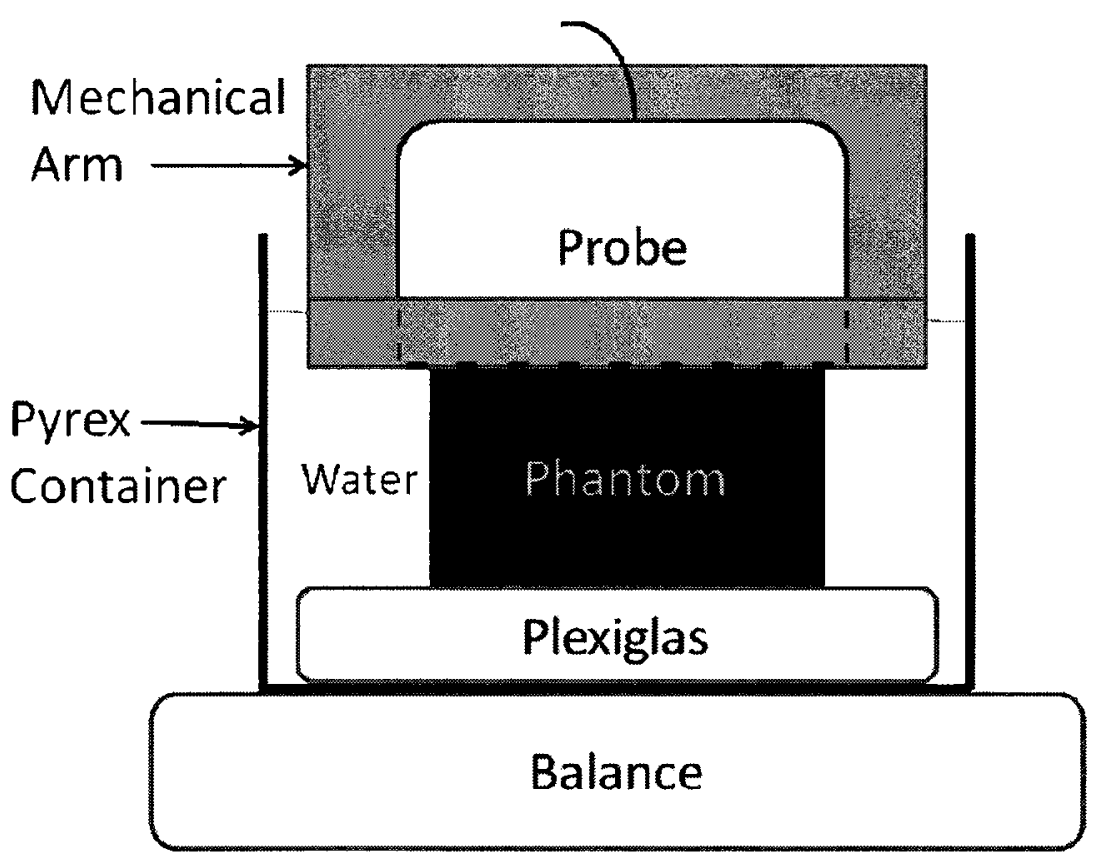

Figure 3.8: Block diagram of Stress-relaxation experimental setup. 


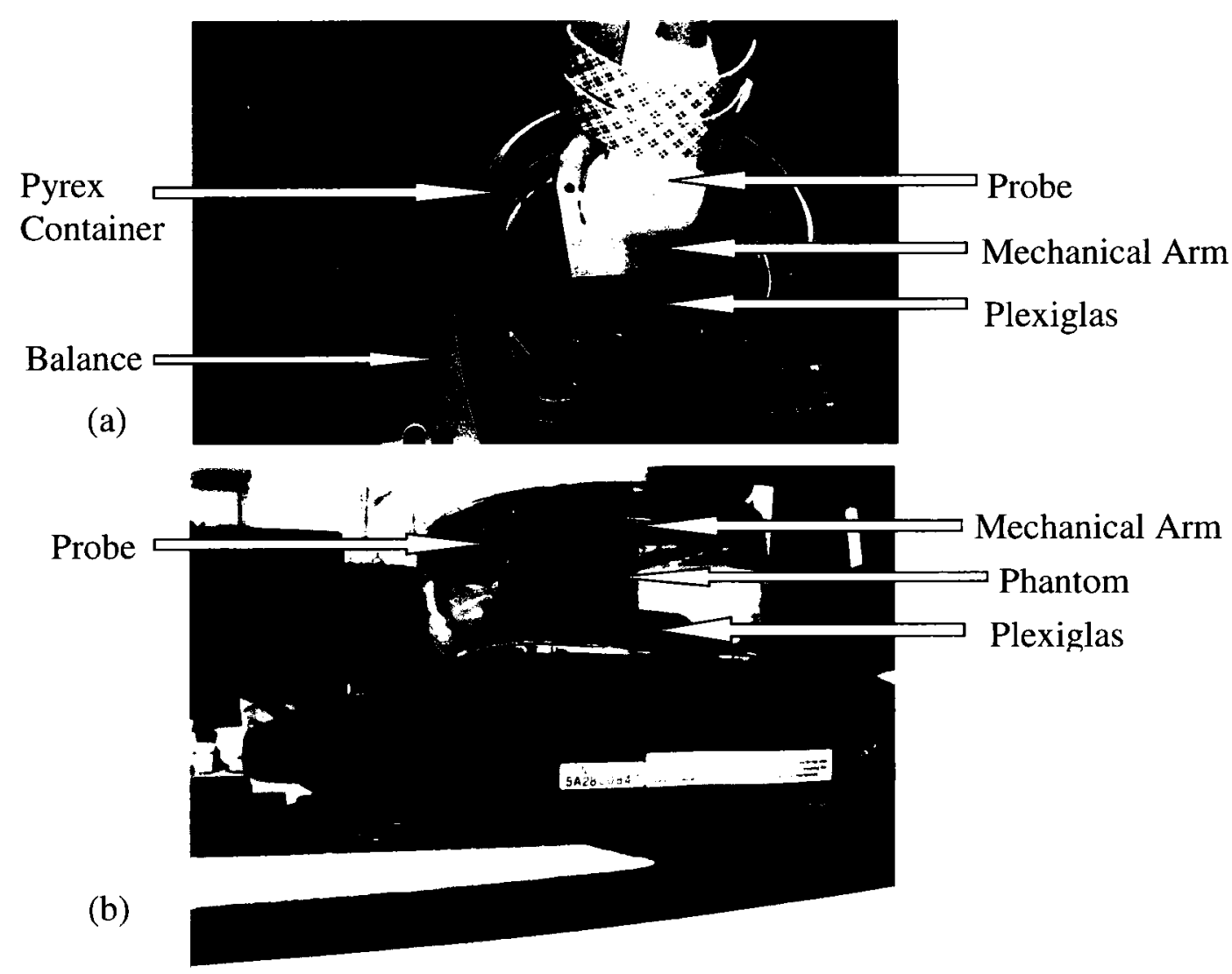

Figure 3.9: Setup with (a) top view of probe, mechanical arm, Pyrex container, Plexiglas, balance and phantom and (b) side view of phantom below mechanical arm and probe and above Plexiglas.

The mechanical arm and probe were lowered into the water until the bottom of the mechanical arm was near but not touching the phantom. The arm was then lowered in steps of $0.06 \mathrm{~mm}$ until the rate of weight change on the balance was greater than the rate of weight change with just water beneath the arm, as shown in Fig. 3.10. At this depth contact was considered achieved, and thus the balance was zeroed. Recording of weight started and continued at $1 \mathrm{~s}$ intervals. The mechanical arm was then lowered $0.2 \mathrm{~mm}$ at $2000 \mathrm{steps} / \mathrm{s}$, or $12 \mathrm{~mm} / \mathrm{s}$, and the $0.2 \mathrm{~mm}$ phantom compression was maintained for 5 minutes. The weight recording stopped and the arm was raised $0.2 \mathrm{~mm}$ at the same rate. 
The phantom was then allowed to rest for $10 \mathrm{~min}$, which was twice the compression duration. The procedure was then repeated for $0.4 \mathrm{~mm}, 0.6 \mathrm{~mm}, 0.8 \mathrm{~mm}, 1.0 \mathrm{~mm}$ and 1.2 $\mathrm{mm}$ total compressions. Since $\sigma=m g / a$, where $m$ is mass, $g$ is gravity and $a$ is area, the stress on the material can be calculated once the weight, or mass, and the area are known.

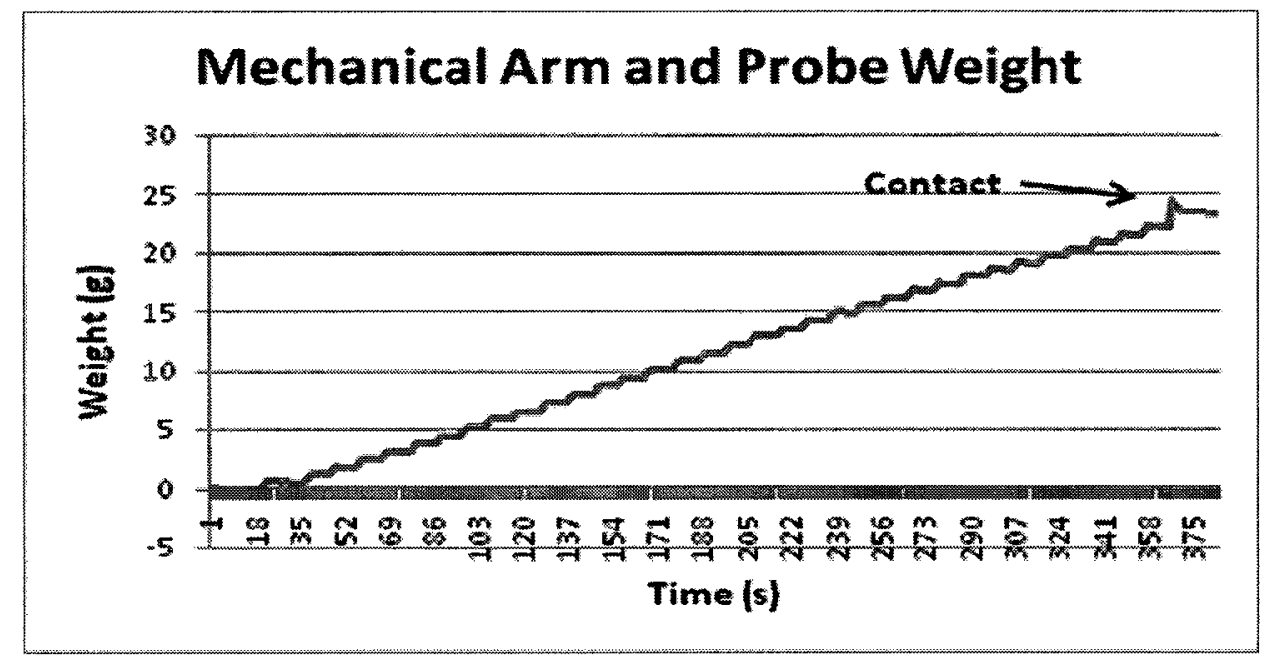

Figure 3.10: Plot of mechanical arm and probe displacement weight during contact test.

In order to determine the stress on the phantom, the weight due to the arm and probe immersed in water would need to be subtracted post-processing. To determine this weight, the phantom was moved from underneath the arm with a metal hex wrench (which had an optimal shape to get under the arm to move the phantom and because of its smoothness water was not removed from the bowl) and kept below water. The mechanical arm was then lowered $0.2 \mathrm{~mm}$, held for $30 \mathrm{~s}$ then raised $0.2 \mathrm{~mm}$. The results of this part of the experiment are shown in Fig. 3.11. The weight subtracted for the 0.2 $\mathrm{mm}$ compression was $2.4 \mathrm{~g}$. This procedure was repeated for $0.4 \mathrm{~mm}, 0.6 \mathrm{~mm}, 0.8 \mathrm{~mm}$, 
$1.0 \mathrm{~mm}$ and $1.2 \mathrm{~mm}$ compressions. Once the data were collected for the 2 -w\% agar phantom the procedure was repeated for the 3-w\% agar phantom.

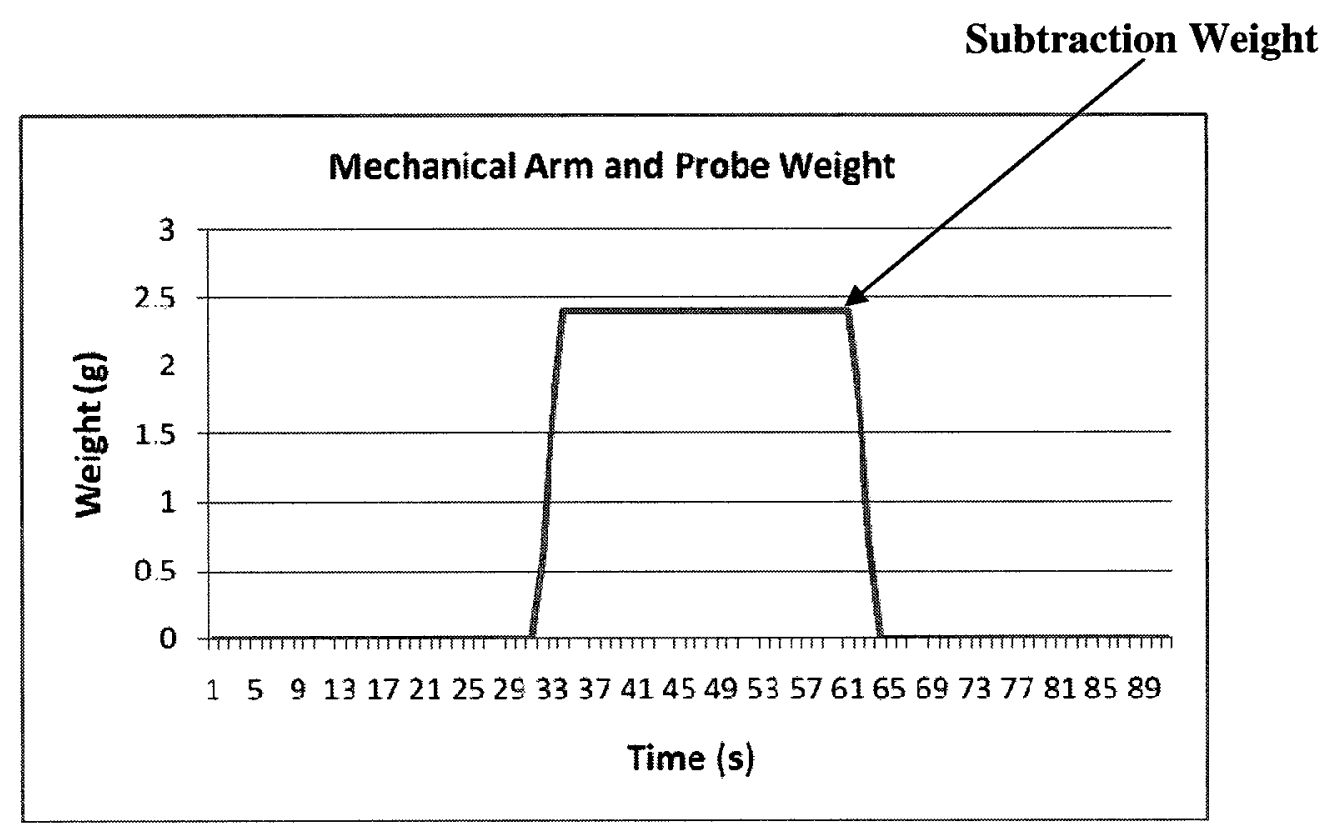

Figure 3.11: Plot of mechanical arm and probe displacement weight with $0.2 \mathrm{~mm}$ compression.

\subsubsection{Young's Modulus}

Young's modulus, E, of a material is governed by Hooke's law:

$$
E=\frac{\sigma}{\varepsilon}
$$

where $\sigma$ and $\varepsilon$ are stress and strain, respectively. This relationship holds in the elastic region of a stress-strain curve. Stress is:

$$
\sigma=\frac{f}{a}
$$

where $f$ is force and $a$ is area over which the force is applied. Since $f=m \cdot g$, stress is also: 


$$
\sigma=\frac{m g}{a}
$$

where $m$ is mass and $g$ is gravity. Strain is the deformation of a material, given by

$$
\varepsilon=\frac{\Delta z}{z}
$$

where $\Delta z$ is the change in length after compression or tension is applied and $z$ is the original length. Substituting eqn. 3.5 and 3.6 into 3.3 Young's modulus of a material is:

$$
E=\frac{m g}{a} \frac{z}{\Delta z}
$$

which has units of pressure $\left(\mathrm{Pa}\right.$ or $\left.\mathrm{Nm}^{-2}\right)$. Isotropic materials have the same mechanical properties in all directions, thus also have the same Young's modulus in all directions.

In order to determine Young's modulus from experimental results a phantom is compressed with a predetermined displacement over a predetermined area and the weight, or mass, of phantom is recorded, as described in section 3.2.2. The Young's modulus of the material can then be determined using the slope of the stress-strain curve, as shown in Fig. 3.12 (a), section 3.2.4.

\subsubsection{Hysteresis}

Elastic material stress-strain curves measured during load and unload do not change, as shown in Fig. 3.12 (a). However, when biological tissues are strained, or loaded, and unloaded the stress-strain curves for the load and unload are not the same, as shown in Fig. 3.12 (b) [26]. This phenomenon is called hysteresis. It was desired to understand if agar phantoms, which were created to resemble soft tissue, exhibit 
hysteresis. In addition, phantoms that exhibit hysteresis could affect Young's modulus calculations. A phantom that exhibits hysteresis (different stress-strain slopes) could have different Young's modulus results depending on experimental conditions, ie. load versus unload, used. Therefore, it is important to select and explain the experimental conditions used to create stress-strain curves and calculate Young's modulus.
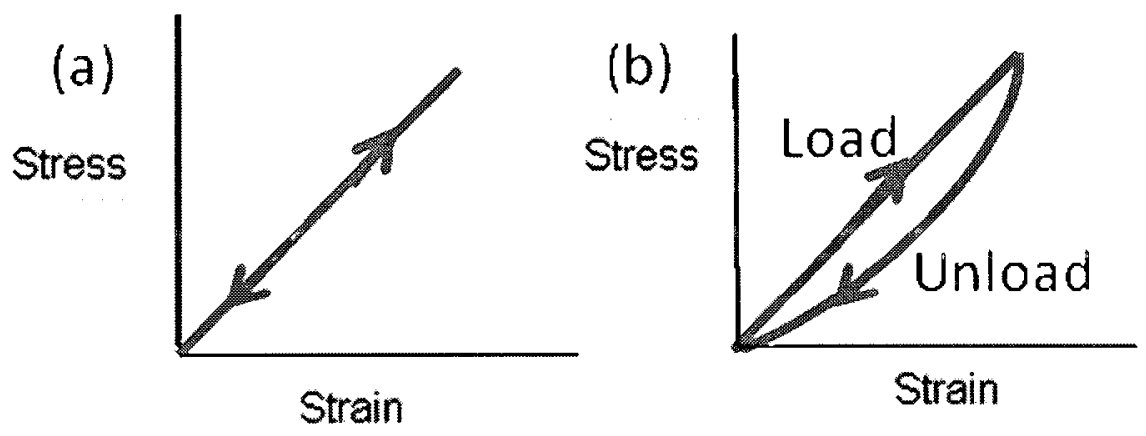

Figure 3.12: Stress-strain curves for (a) elastic and (b) biological materials.

Unlike the stress-relaxation experiments, the hysteresis measurements were done in air and not water, as shown in Fig. 3.13. It was determined after hysteresis measurements were completed that the addition of water would prevent phantom dehydration and reduce slip boundary conditions. Contact was achieved by observing a rapid weight change, $>1 \mathrm{~g}$, on the scale. Using the mechanical arm the phantom was compressed, or loaded, from zero to $1.2 \mathrm{~mm}$ at $0.03 \mathrm{~mm} / \mathrm{s}$ and unloaded at the same rate. The process was repeated 5 times (a total of 6 cycles) using auto-reverse (for unloads) and repeat (5 cycles) with the mechanical stage software, Cosmos. Balance (EJ-4100, 
A\&D Company, Tokyo, Japan) weight was recorded every 0.1s using RSWeight software provided by the manufacturer.

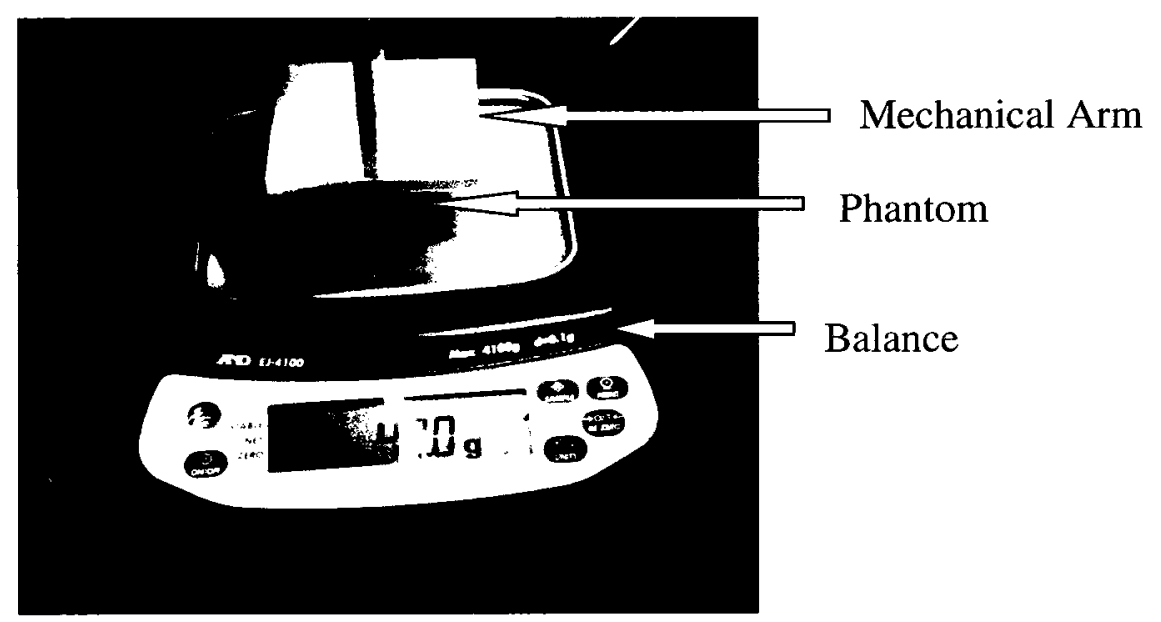

Figure 3.13: Picture of hysteresis measurement setup.

\subsection{Tissue Mimicking Phantom Characterization Results and Discussion}

\subsubsection{Ultrasound Velocity}

Fig. 3.14 shows the ultrasound signals obtained with (a) water as a reference, (b) with the phantom and (c) with the phantom with the $\mathrm{Al}$ foil reflector. The foil reflector at the water/phantom interface improved the SNR of the $V_{3}$ echo, as seen in Fig. 3.14 (c), of which the SNR was $14 \mathrm{~dB}$, while the $\mathrm{V}_{3}$ echo was not clearly observed in Fig. 3.14 (b) without the foil reflector. The SNR was calculated $20 \cdot \log$ of the peak signal amplitude divided by the mean baseline signal ( 32 data points). The $V_{3}$ echo in Fig. 3.14 (b) was probably from the scatterers in the phantom rather than the water/phantom interface. 
Therefore, it may be difficult to accurately determine the time of the peak amplitude of the $V_{3}$ echo in Fig. 3.14 (b).

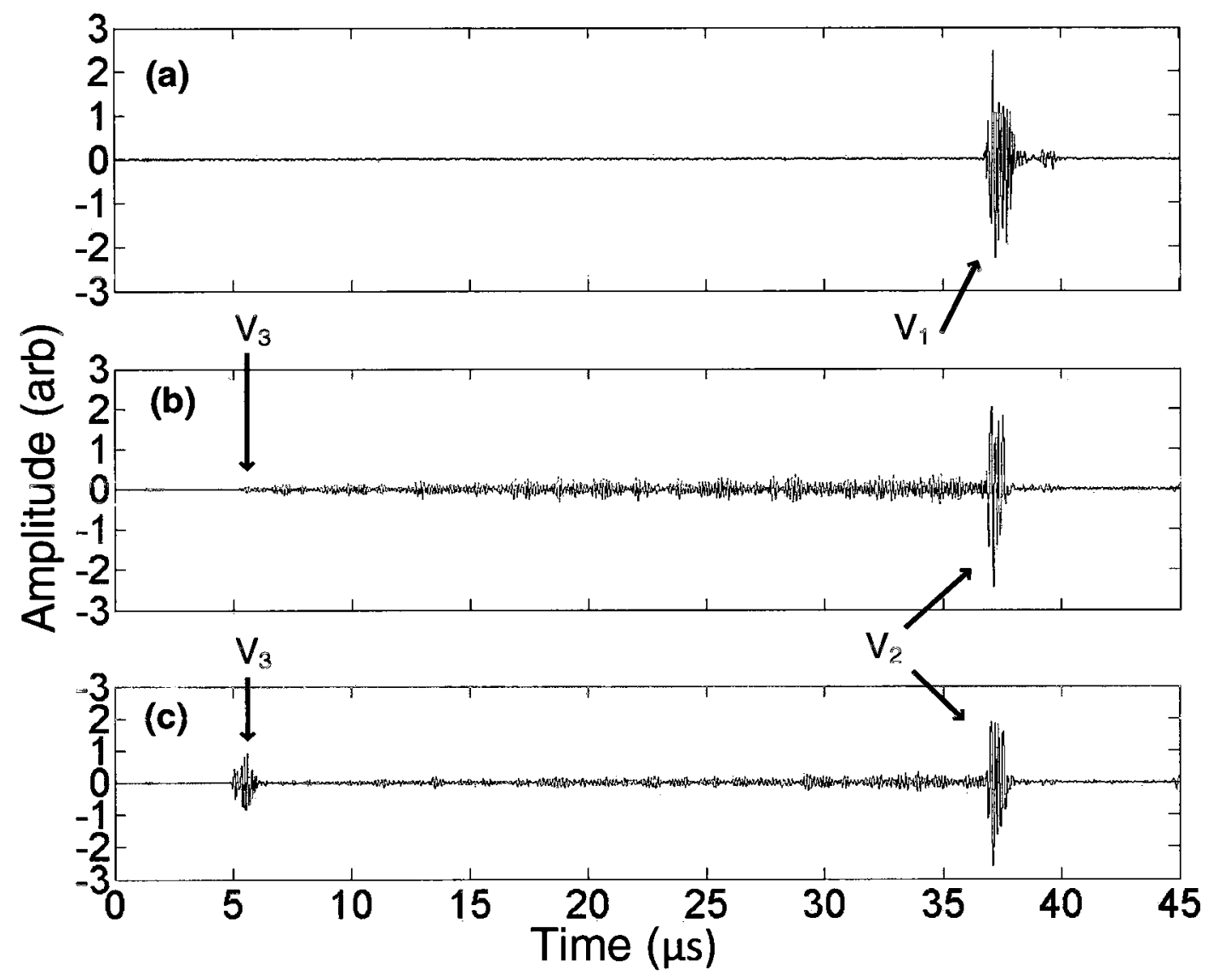

Figure 3.14: Ultrasound signals acquired: (a) without phantom, (b) with phantom; and (c) with phantom and foil reflector.

The ultrasound velocities of five phantoms with varying agar concentrations were measured at room temperature with the $\mathrm{Al}$ foil reflector. The results, calculated using eqn. 3.2 from section 3.2.1, are shown in Fig. 3.15. The measurement reproducibility was estimated within $1.5 \mathrm{~m} / \mathrm{s}(0.1 \%)$ from 10 repeated measurements using a 2-w\% agar phantom without graphite powder. The measured result shown in Fig. 3.15 did not exhibit 
a significant velocity differences in the agar concentration range between 1-w\% and 3w\% with our measurement reproducibility of $1.5 \mathrm{~m} / \mathrm{s}$.

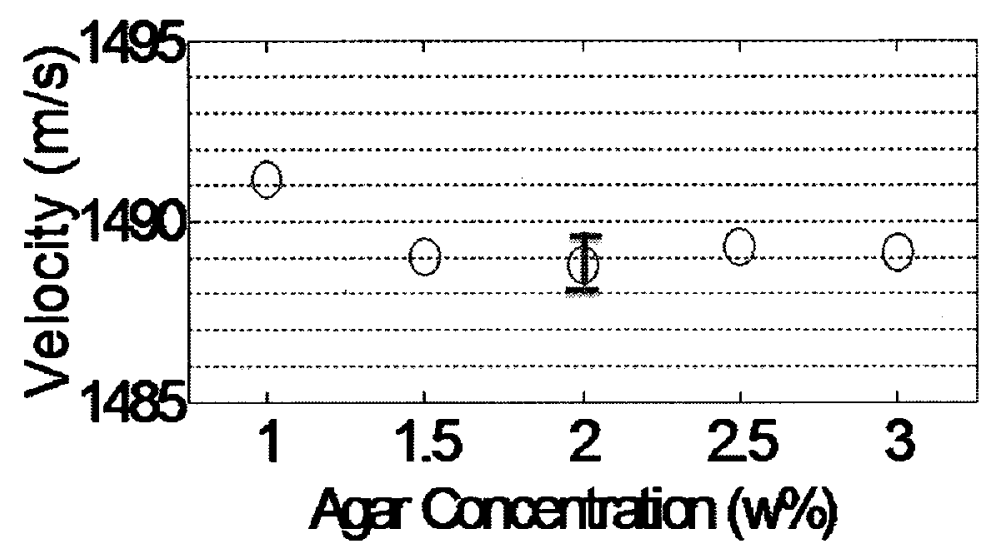

Figure 3.15: Ultrasound velocities of phantoms measured with $\mathrm{Al}$ foil reflector with respect to agar concentration.

For the velocity measurement in Fig. 3.15, obtained by using eqn. 3.2, the effect of the $\mathrm{Al}$ foil reflector on the velocity measurement was not considered, which could cause a velocity measurement error. The $\mathrm{V}_{3}$ echo observed in Fig. 3.14 (c) may be composed of echoes reflected from the top and bottom of the $\mathrm{Al}$ foil. Note that the time shift between the top and bottom echoes from the $\mathrm{Al}$ foil was $4.67 \mathrm{nsec}$ with the $\mathrm{Al}$ foil thickness of $15 \mu \mathrm{m}$ and the velocity in $\mathrm{Al}$ of $6420 \mathrm{~m} / \mathrm{s}$ [28], thus, these echoes could overlap in the time domain.

In order to investigate the effect of $\mathrm{Al}$ foil thickness on the velocity measurement, it is assumed that the echo reflected from the top of the $\mathrm{Al}$ foil was dominant in the $\mathrm{V}_{3}$ echo. In such case, the velocity of the phantom, $c_{p}$, can be obtained using eqn. 3.8 by 
considering the $\mathrm{Al}$ foil in the measurement model, where $d_{f}$ and $c_{f}$ are the thickness and velocity of the $\mathrm{Al}$ foil reflector, respectively. The $\mathrm{V}_{2}$ echo passes through the $\mathrm{Al}$ foil in addition to the phantom. Therefore, the velocity error $\Delta c_{p}$ is defined as eqn. 3.9.

$$
\begin{aligned}
& c_{p}{ }^{*}=c_{w}\left[1-\left(\frac{\Delta t_{1}+2 d_{f}\left(\frac{1}{c_{f}}-\frac{1}{c_{w}}\right)}{\Delta t_{\mathrm{z}}+\frac{2 d_{f}}{c_{f}}}\right)\right] \\
& \Delta c_{p}=\frac{\left(c_{p}-c_{p}\right)}{c_{p}} \times 100
\end{aligned}
$$

Fig. 3.16 presents the results of velocity error, $\Delta c_{p}$, calculated using eqn 3.8 and 3.9. For an $\mathrm{Al}$ foil reflector with $15 \mu \mathrm{m}$ thickness, the error in the ultrasound velocity was $0.05 \%$ or $0.7 \mathrm{~m} / \mathrm{s}$ for an ultrasound velocity of $1490 \mathrm{~m} / \mathrm{s}$. The error was smaller than our measurement reproducibility of $1.5 \mathrm{~m} / \mathrm{s}$ under our experimental conditions employed.

The ultrasound velocity without the foil was determined to be $1489 \mathrm{~m} / \mathrm{s}$ (but the peak was manually selected), while the velocity with the foil measured $1490 \mathrm{~m} / \mathrm{s}$. Although there was not much difference in the velocity measurement, the correlation coefficient using the foil target increased significantly from 0.018 to 0.2 . To obtain the same velocity error $(0.05 \%)$ using callipers the calliper measurement accuracy would need to be $0.1 \mathrm{~mm}$ under our experimental conditions, which could be difficult to accomplish due to the softness and deformability of the sample. 


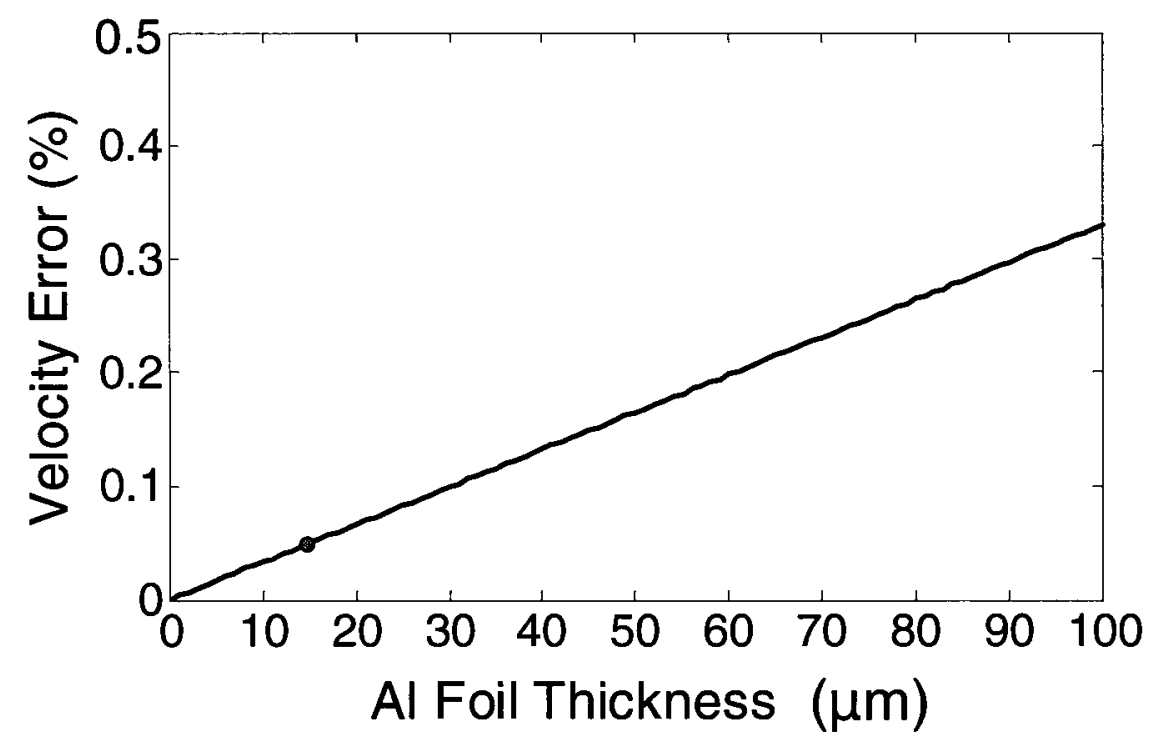

Figure 3.16: Velocity error with respect to $\mathrm{Al}$ foil thickness.

\subsubsection{Stress-Relaxation}

There is stress-relaxation in the phantom, which also exists in biological tissue, because under constant strain the internal stress in the phantom is not constant, but gradually reduces, ie. relaxes. As the applied strains increase the internal stress in the material increased for the 2 and 3-w\% agar phantoms, as shown in Fig. 3.17 (a) and (b), respectively. In addition, there is greater internal stress on the stiffer 3-w\% agar phantom compared to the 2-w\% agar phantom at each compression, as shown in Fig. 3.18 (a) through (c) and Fig. 3.19 (a) through (c). This result was as expected because it requires more force, or stress, to deform stiffer materials than softer, or less stiff, materials. 


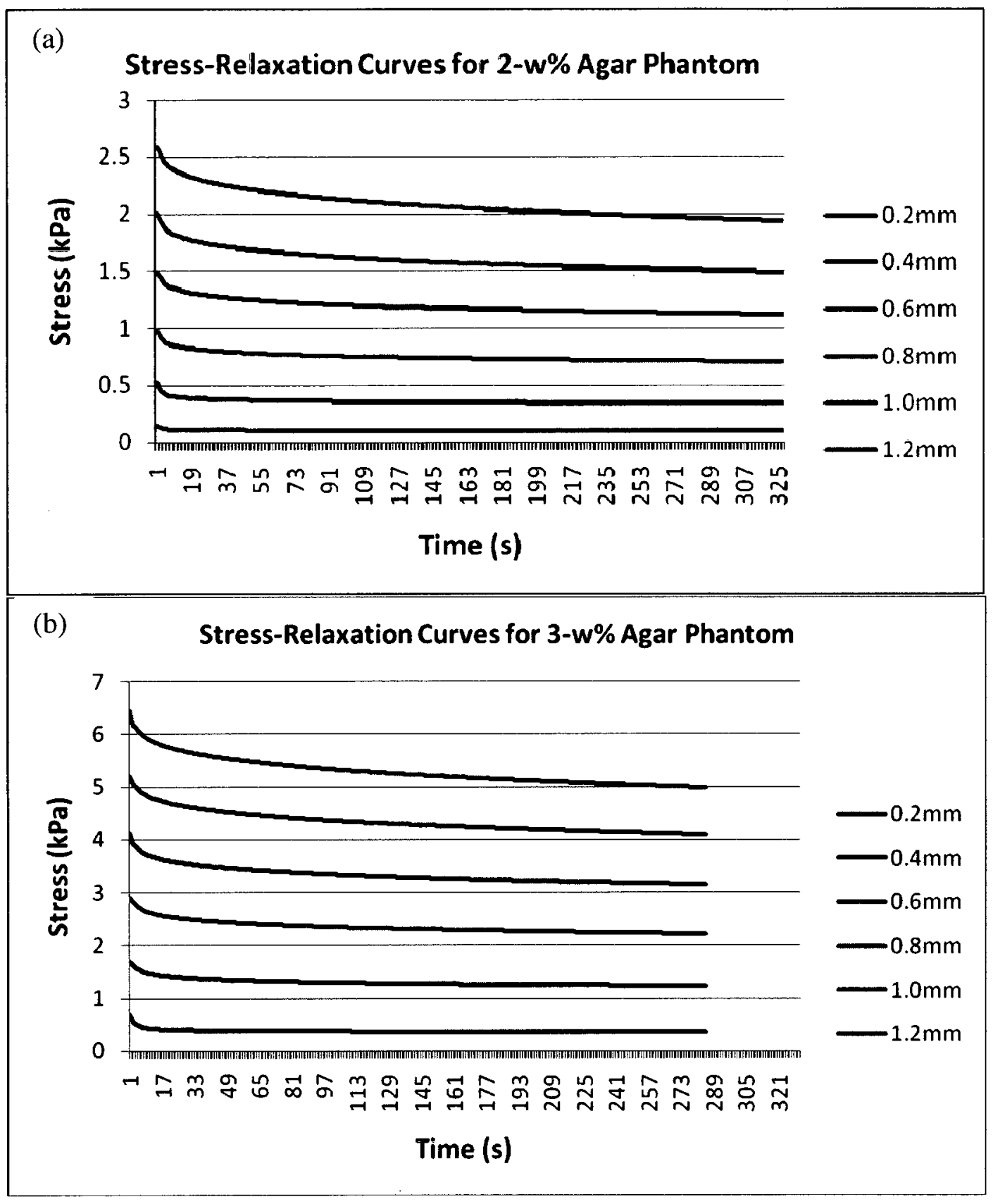

Figure 3.17: Stress-relaxation curves for (a) 2 and (b) 3-w\% agar phantoms. 
(a)

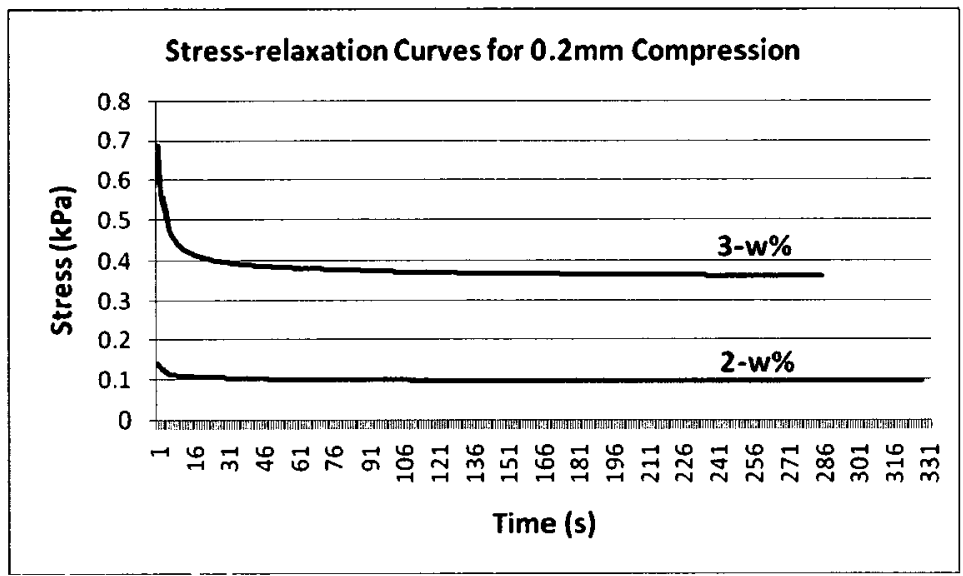

(b)

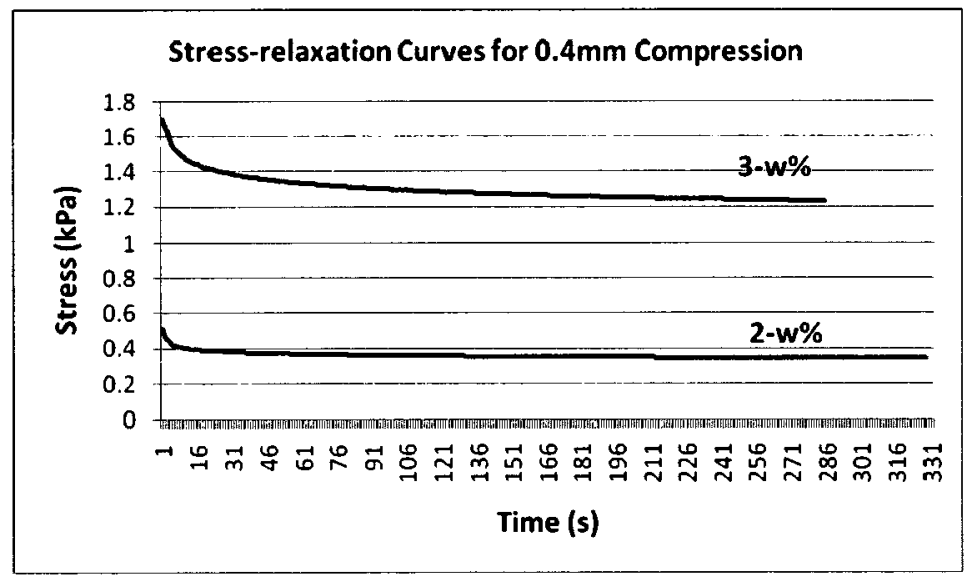

(c)

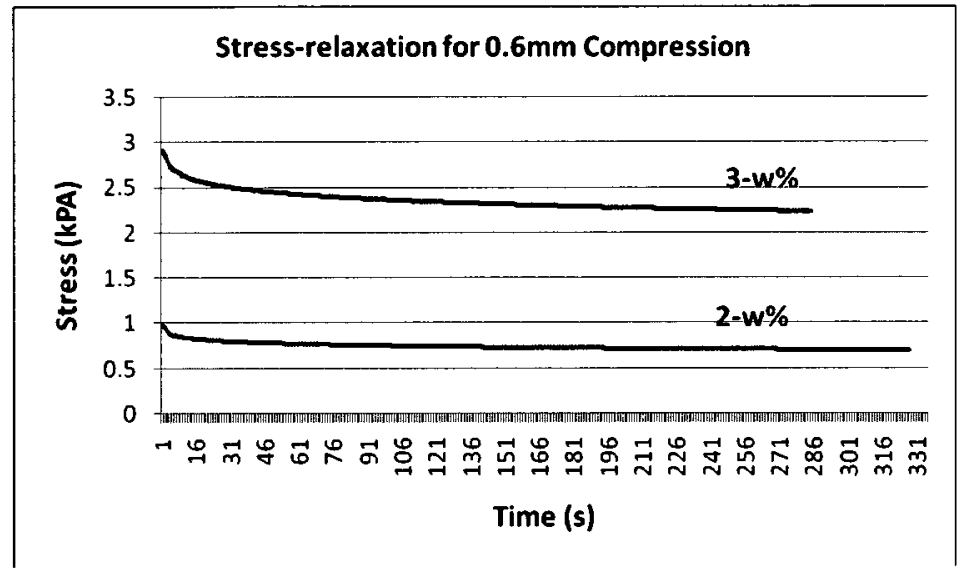

Figure 3.18: Stress-relaxation curves for $2 \mathrm{~g}$ and $3 \mathrm{~g}$ agar phantoms with (a) $0.2 \mathrm{~mm}$, (b) $0.4 \mathrm{~mm}$ and (c) $0.8 \mathrm{~mm}$ compression. 
(a)

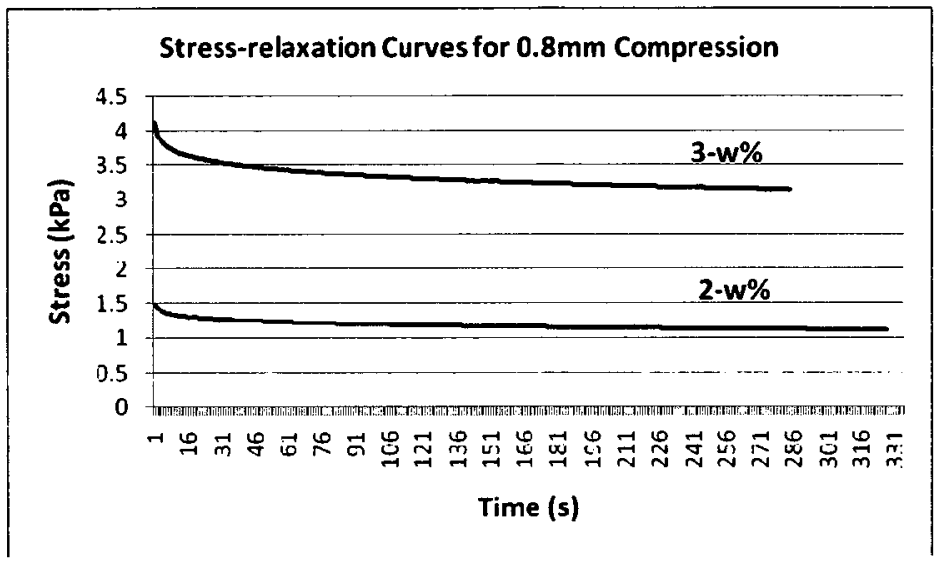

(b)

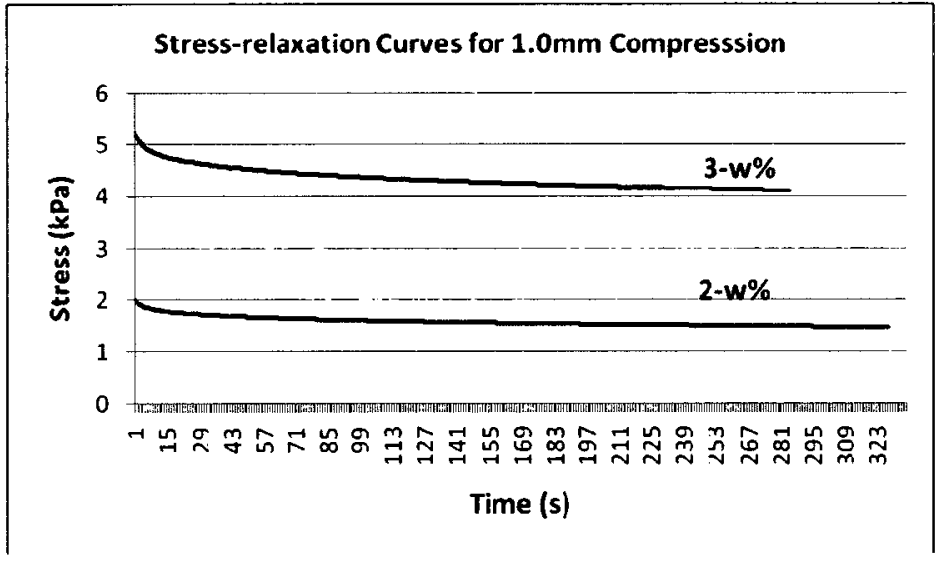

(c)

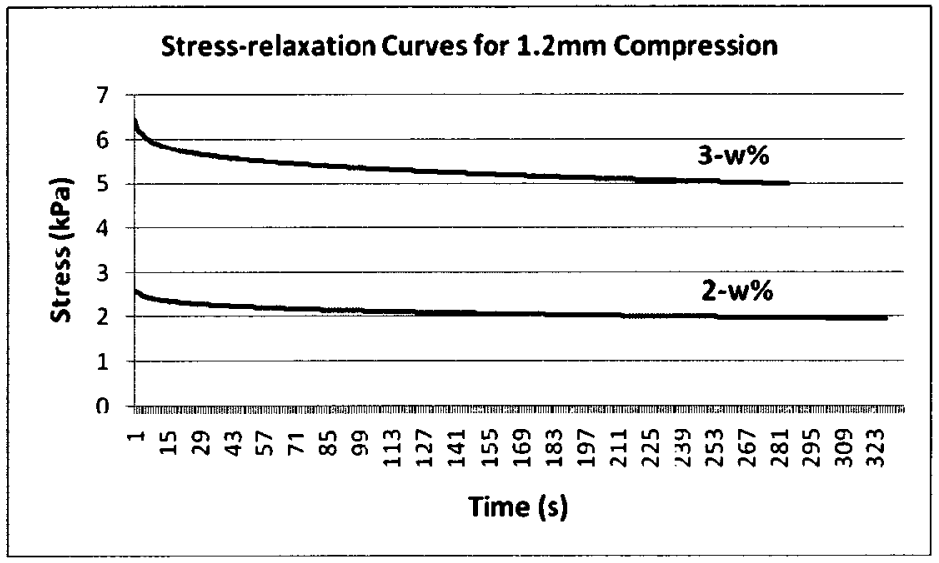

Figure 3.19: Stress-relaxation curves for $2 \mathrm{~g}$ and $3 \mathrm{~g}$ agar phantoms with (a) $0.8 \mathrm{~mm}$, (b) $1.0 \mathrm{~mm}$ and (c) $1.2 \mathrm{~mm}$ compression. 
To detect a tumor, contrast between the tumor and surrounding material must exist. Tumor contrast, $\mathrm{C}$, can be calculated using [1]:

$$
C=\frac{\left(\sigma_{t}-\sigma_{b}\right)}{\sigma_{b}}
$$

where $\sigma_{t}$ and $\sigma_{b}$ are the stress of the tumor and background, or surrounding tissue, respectively. Contrast was calculated using eqn. 3.10 for increasing strains, as shown in Fig. 3.20, using the maximum stress (peaks of stress-relaxation curves) at each strain for the $3-\mathrm{w} \%$ and $2-\mathrm{w} \%$ phantoms. The $3-\mathrm{w} \%$ and $2-\mathrm{w} \%$ phantoms were used to represent the tumor and background, respectively. Fig. 3.20 shows that contrast exists at all applied compressions. In addition, as the applied strain increased the contrast between the tumor and the surrounding area decreased.

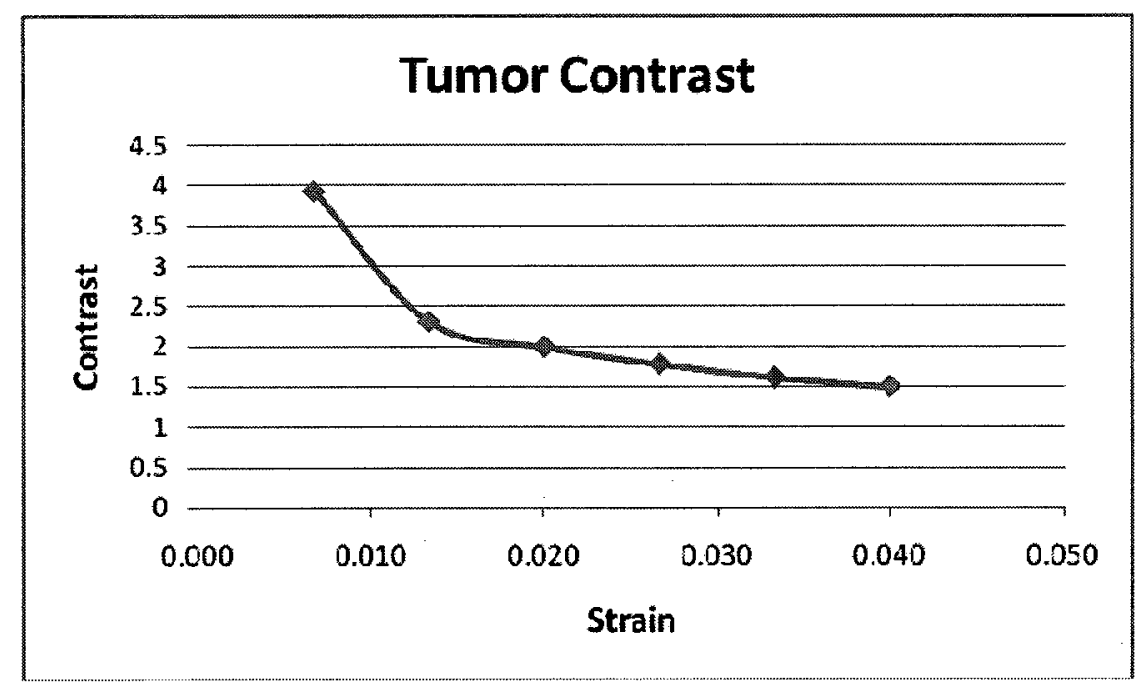

Figure 3.20: Tumor contrast with increasing strain. 


\subsubsection{Young's Modulus}

Because of the stress-relaxation in the phantom, the Young's modulus of the material will depend on when the stress is measured. To improve the accuracy of the Young's modulus calculations, peak stress measurements for each strain were used in this thesis. It is also possible to calculate Young's modulus using stress measurements when the time constant of the stress-relaxation curve is reduced, perhaps after 10min.

Fig. 3.21 shows the stress-strain curves using the peaks of the stress-relaxation curves for increasing strain from section 3.3.2. Since the slope of the 3-w\% agar phantom is greater than the slope of the $2-w \%$ agar phantom, the $3-w \%$ agar phantom is stiffer than the 2-w\% agar phantom. This is as expected since the 3-w\% agar phantom felt stiffer to touch than the 2-w\% agar phantom.

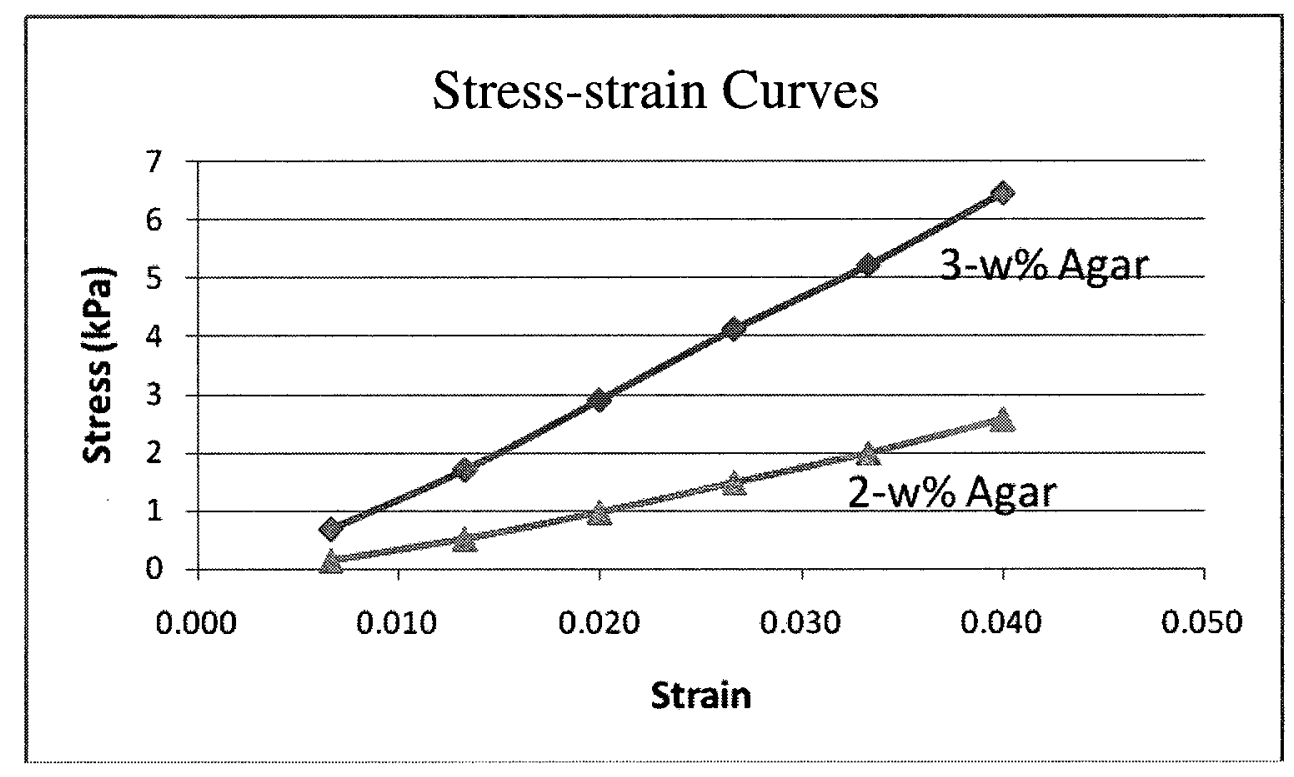

Figure 3.21: Stress-strain curves. 
The original measurement data for the $2-\mathrm{w} \%$ and $3-\mathrm{w} \%$ stress-strain curves, as shown in Fig. 3.21, and the linear fit for the stress-strain curves are shown in Fig. 3.22. The Young's modulus of the 2-w\% and 3-w\% agar phantoms were determined to be 74 $\mathrm{kPa}$ and $174 \mathrm{kPa}$, respectively. Since strain is dimensionless and stress has units of pressure $(\mathrm{kPa})$, Young's modulus also has units of pressure. The Young's modulus results will be discussed in Chapter 4, section 3.5.2 and in the Conclusion.

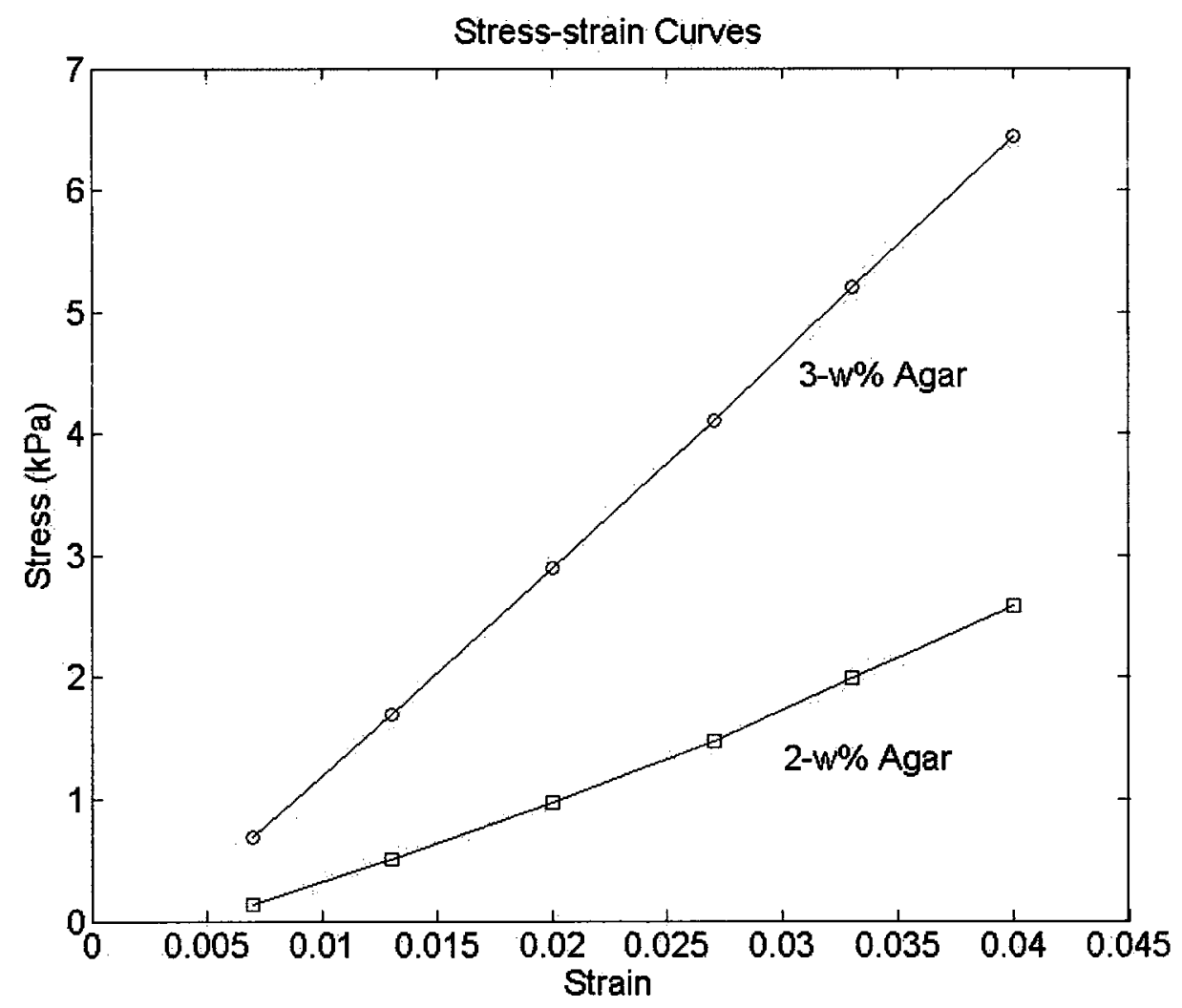

Figure 3.22: Original (markers) and linear fit (solid line) for 2-w\% and 3-w\% agar phantom stress-strain curves.

\subsubsection{Hysteresis}

The balance weight $(\mathrm{g})$ or mass recordings collected were converted to stress $(\mathrm{Pa})$, normalized and plotted in Fig. 3.23. In addition, to produce the plot in Fig. 3.23 a 
threshold of $30 \mathrm{~g}$, or $327 \mathrm{~Pa}$, was also selected. Since there was very little variation from the first and the other cycles, the stress results from the first cycle are provided.

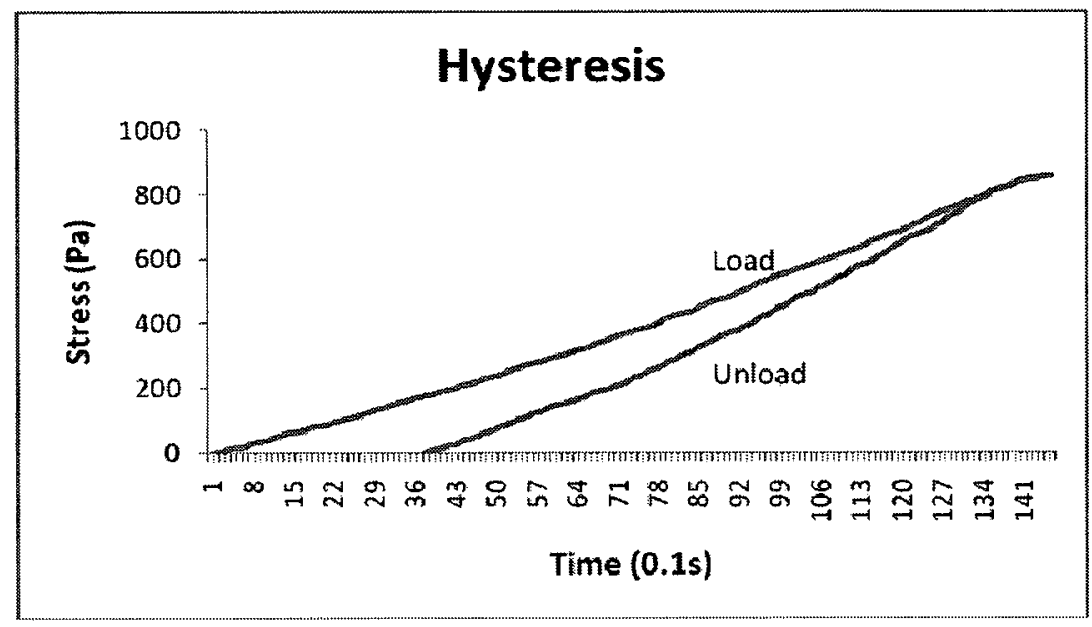

Figure 3.23: Hysteresis for $2 \mathrm{w} \%$ agar phantom.

The change in stress seen in the phantom during load and unload were not the same, as seen by the different slopes in Fig. 3.23. The phantoms stress-strain curves will depend on whether the phantom is being loaded or unloaded. The unload curve does not converge with the load curve near time zero, indicating that energy is stored in the system. Because a threshold was chosen it can not be determined at this time, based on the hysteresis plot, if the material behaves as elastic or inelastic at low compressions. 


\section{Chapter 4: \\ Elastic Imaging}

\subsection{Displacement and Strain Measurement Methods}

\subsubsection{Phantom Construction}

Isotropic, single concentration (uniform) phantoms with 2 and 3 w\% agar were constructed, as discussed in Chapter 3, section 3.1, for elastic imaging. In addition, composite phantoms to resemble a tumor were also constructed for elastic imaging, as shown in Fig. 4.1 (c). An outline and flowchart of the construction methods for uniform and tumor phantoms is located in Appendix A. Silicone molds were modified, as shown in Fig 4.1 (a) using two plastic tubes, outer diameter $9.9 \mathrm{~mm}$, glued to the center of the silicone molds. These molds are referred to as tumor molds. Phantoms were constructed with 2-w\% agar backgrounds and 3-w\% agar centers for elastic imaging. The procedure involved constructing 2-w\% agar phantoms for the background, as discussed in Chapter 3, section 3.1, using the modified tumor molds and then the following additional steps were performed to construct the stiffer center, or tumor, of the phantom.

The background phantoms were allowed to sit in an ice water bath for a minimum of one hour and then unmolded and placed into a silicone uniform mold without a plastic tube. The holes at the bottom of the phantoms were now covered and the phantoms were easier to hold for the next steps. A 3-w\% agar phantom was constructed using the same 
procedure used to construct the 2-w\% agar background phantoms, except that the mixture was poured into the hole of the 2 -w\% agar background phantoms. The mixture was slowly poured into the hole while the background phantoms were tilted. Slowly pouring and tilting the phantoms allowed the mixture to flow easier to the bottom of the hole and reduced the risk of trapping air bubbles. The molded tumor phantom is shown in Fig. 4.1 (b).

(a)

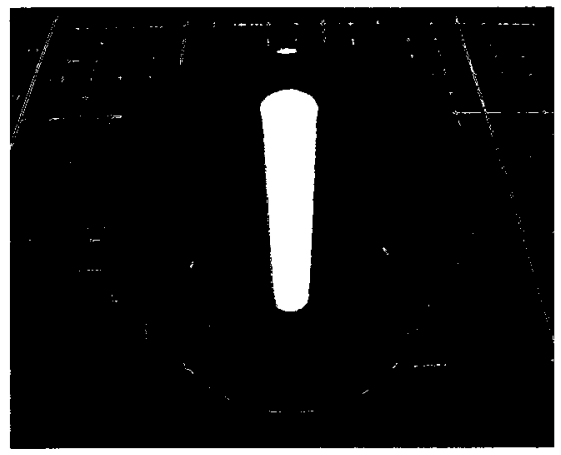

(b)

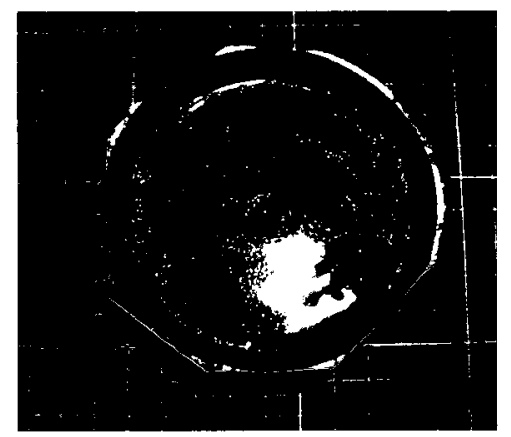

(c)

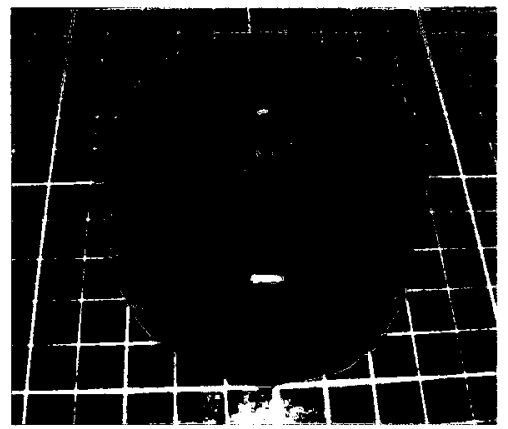

Figure 4.1: Pictures of (a) silicone tumor mold with plastic tube, (b) tumor phantom in uniform mold and (c) unmolded phantom.

All phantoms (single and composite) used for elastic imaging were unmolded and sliced uising a device composed of two blades separated in parallel by $30 \mathrm{~mm}$ spacers, as shown in Fig. 4.2 (a). A sliced tumor phantom is shown in Fig. 4.2 (b). When not being 
used all phantoms for elastic imaging were stored at room temperature in silicone molds, covered with plastic wrap and placed into a Pyrex container with distilled water to prevent dehydration.

(a)

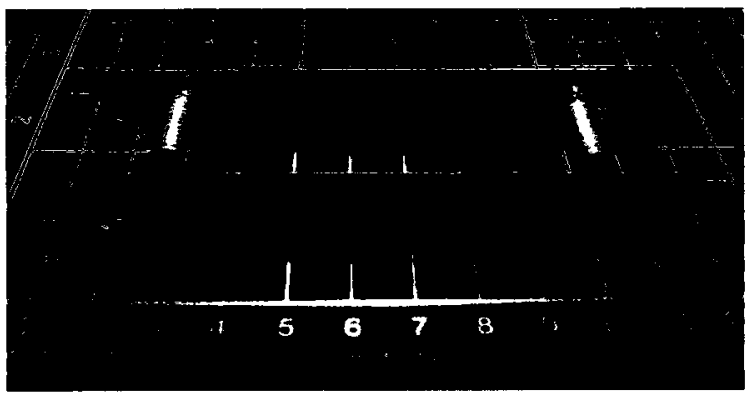

(b)

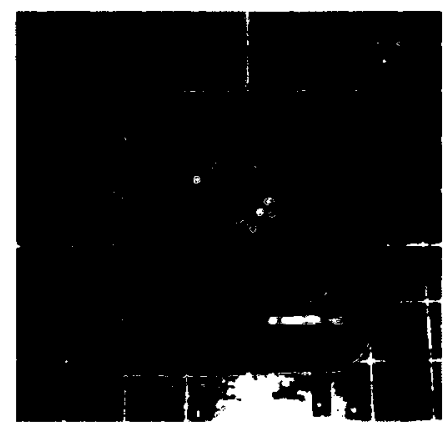

Figure 4.2: Pictures of (a) device used to slice phantoms for elastic imaging and (b) sliced composite, tumor phantom.

\subsubsection{Displacement and Strain Imaging}

Elastography, or strain imaging, of materials can be explained using a spring model [29]. When uniform springs are compressed with a uniaxial load the strain profile, as shown in Fig. 4.3 (a), is constant [29]. As shown in Fig. 4.3 (b), the strain profile changes when a less compressible, or stiff, spring is sandwiched between two equally more compressible, or weak, springs [29]. Less compressible tissue, such as a stiff tumor, surrounded by more compressible tissue, would have a similar strain profile to Fig. 4.3 (b). The strain on the incompressible tissue changes relative to the surrounding compressible tissue. 

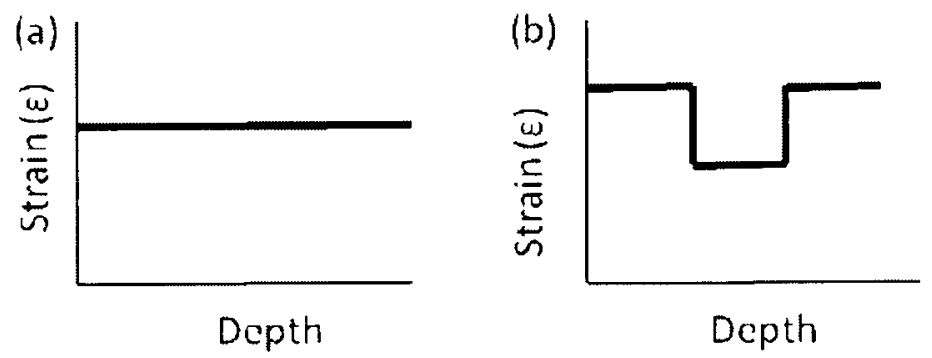

Figure 4.3: Strain profile for (a) uniform springs and (b) unequal springs.

When a uniform, or homogeneous, phantom is compressed with a uniaxial load, in the direction of beam propagation, the longitudinal displacement and strain are seen at all points in the material [12]. Since strain is the gradient of displacement, a uniform phantom would have a linear displacement with increasing depth, as shown in Fig. 4.4. Displacement increases with phantom depth, as illustrated with post-compression scatterer displacement in Fig. 4.5, because the probe position is the reference. Thus, it is important to understand the ultrasound coordinate system that governs ultrasound measurement results.

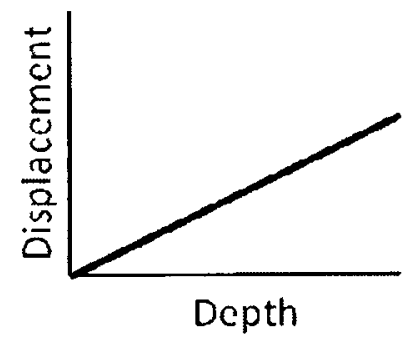

Figure 4.4: Plot of displacement versus phantom depth. 


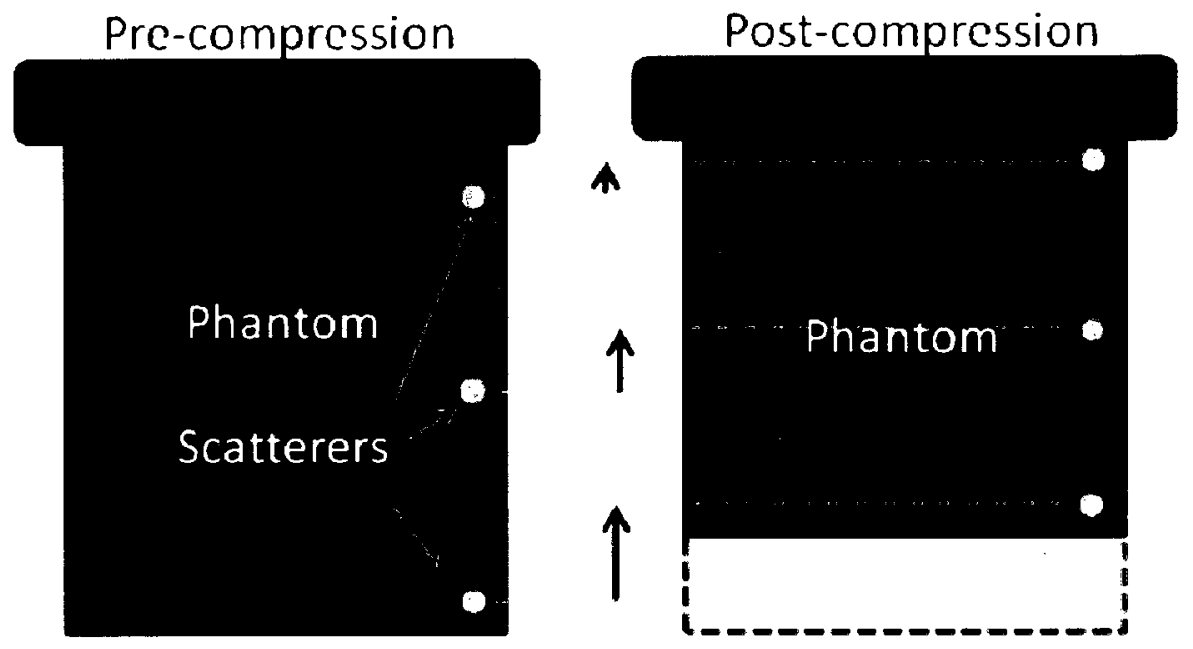

Figure 4.5: Diagram to illustrate scatterer displacement represented with black arrows.

The ultrasound coordinate system defines motion relative to the ultrasonic probe. The frame of reference is the probe, so although the probe has physically moved downward after compression, as shown in Fig. 4.6 (a), the effect of the coordinate system results in positive displacement, as shown in Fig. 4.6 (b). Since the frame of reference is the probe, all movement appears either towards the probe, as shown in Fig. 4.6 (b), or away from the probe, which is the case when the phantom is uncompressed. 


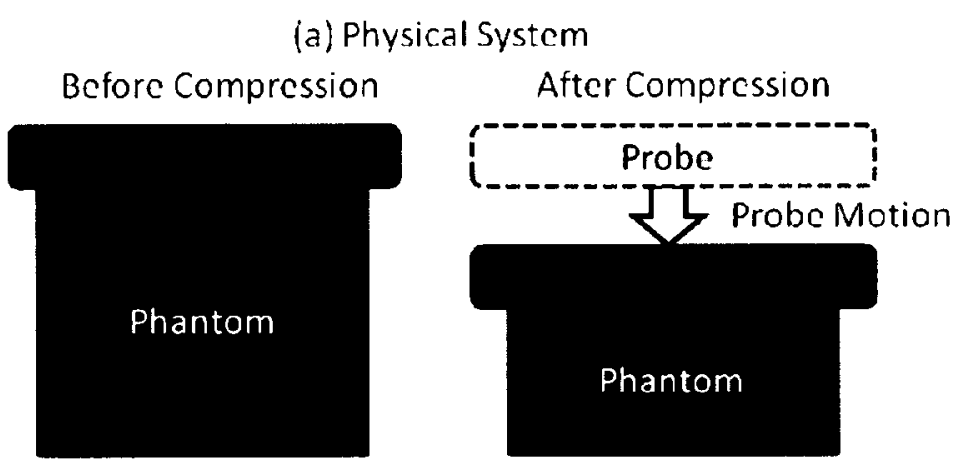

(b) Signal Processing

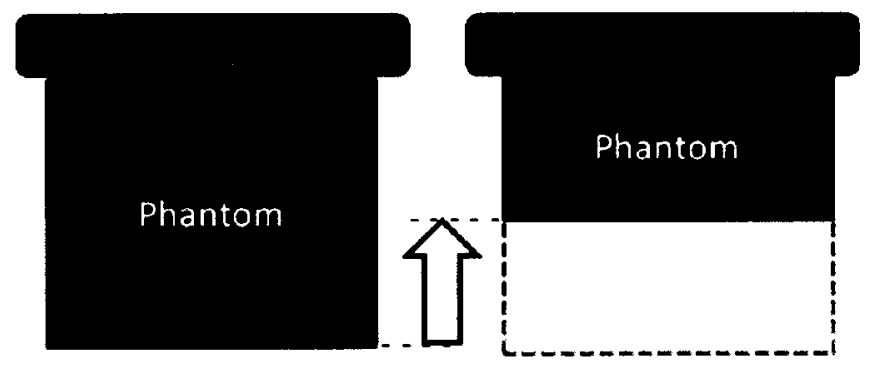

Figure 4.6: Diagrams to illustrate (a) physical system and (b) probe as frame of reference.

The same experimental setup and procedure used for stress-relaxation measurements, described in Chapter 3, section 3.2.2, with the addition of ultrasound RF data acquisition, was also used for displacement and strain imaging. RF signals from scatterers are collected at pre and post-compression conditions, as shown in Fig. 4.7. Although 127 scanlines are collected using B-mode (brightness) modality, for simplicity one scanline RF signal from the pre and post-compression is shown in Fig. 4.7 [30]. 


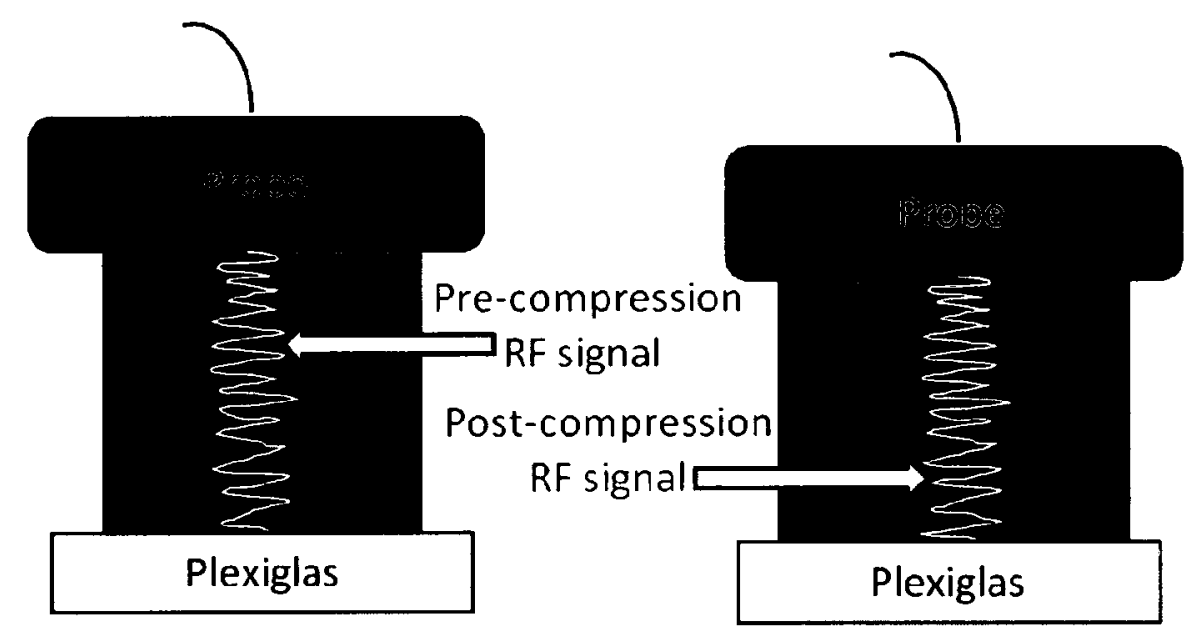

Figure 4.7: Diagram to illustrate RF signals collected for displacement and strain images. Copied from [30].

ART.LAB software (ESAOTE, Maastricht, The Netherlands) on the ultrasound system was used to acquire the RF signals. After contact was achieved the phantom was compressed $0.2 \mathrm{~mm}$ at $12 \mathrm{~mm} / \mathrm{s}$. Once the compression from the mechanical arm stopped, "freeze" was selected on the ultrasound imaging system. Selecting freeze on the imaging system stopped the circular buffer, which holds the past $5.4 \mathrm{~s}$ of event data, from updating. When "acquire" was selected, the data in the circular buffer were then moved to a .zrf file format.

Since the compression rate applied was $12 \mathrm{~mm} / \mathrm{s}$, the time for the phantom to reach $0.2 \mathrm{~mm}$ compression was approximately $0.02 \mathrm{~s}$. Since the ultrasound frame rate using B-mode modality was $30 \mathrm{~Hz}$, the total $0.2 \mathrm{~mm}$ compression was acquired within 1 frame. To ensure that the frames containing the pre- and post-compression were captured, and thus freeze was not selected too slowly, the data in the circular buffer were 
viewed in real-time by selecting "play" in ART.LAB. This procedure was then repeated for $0.4 \mathrm{~mm}, 0.6 \mathrm{~mm}, 0.8 \mathrm{~mm}, 1.0 \mathrm{~mm}$ and $1.2 \mathrm{~mm}$ compressions.

The .zrf file contained the RF signal data, $\mathrm{Z}$ signal data (the envelope of the RF signal), header and other information. When RF data were acquired the contents of the buffer, which contains the past six seconds of events, was dumped directly into memory, thus the frames were in an incorrect chronological order. Temporal reorganization was performed to sort the frames in the correct chronological order for displacement and strain signal analysis. To accomplish this, a Matlab function read.zrf, also provided by the manufacturer, was used to create a matrix (defined as a variable "zsig") that contained the $\mathrm{Z}$ signal data. Using header information in the zsig matrix the time zero frame was identified, and then all of the frames were sorted in the correct chronological order with reference to the time zero frame.

Before RF signals were analyzed the header information and the lens signals, the first 16 and 69 data points, respectively, were removed from the RF signals. In addition to the 85 data points, the water and Plexiglas signals were also removed for displacement and strain imaging.

Phantom compression caused the post-compression RF signals to shift in the time domain. Since scatterer displacement is assumed proportional to time shift, and strain is the gradient of displacement, displacement and strain can be calculated from the time shifts [10]. To determine local displacement and strain at all points, or depths, in the phantom, RF signals are commonly subdivided into windows, as shown in Fig. 4.8. Using correlation of the windowed pre- and post-compression signals, spline 
interpolation and the ultrasound sampling interval $(0.03 \mu \mathrm{s})$ the time delay or shifts, $\tau_{i}$, at each window can be determined.

Correlation is a common method used to estimate signal time delays for strain and displacement imaging [15],[16] . To determine each windows time shift Matlab's built in functions 'xcorr' was used to generate an output correlated signal (amplitudes were correlation coefficients). The peak location, or index, of the windowed auto-correlation of the pre-compression signal provided the windows time zero index (no shift has occurred). Cross-correlation of the windowed pre- and post-compression RF signals also provided an output correlation signal and the index of the peak was determined. However, the sampling interval $(0.03 \mu \mathrm{s})$ was not always sufficient to detect time shifts and the peak determined was not always the best estimate of the peak location, so spline interpolation was performed to improve the accuracy of estimating peak location. Improving the accuracy of the peak location improved time shift accuracy, and thus displacement and strain accuracy.

Spline interpolation was performed using Matlab's built in function 'spline,' which applies a cubic (third order) spline to connect data points. Cubic splines are preferred for this research as they have a smoothing affect at the peak data point (slope does not change rapidly as with linear splines). To improve processing time the entire length of the cross-correlation signal was not splined, but rather the data points before and after the peak data point were selected as end points (series of three data points), and 200 intervals were used between end points. The index of the peak of the splined crosscorrelation was subtracted from the index of the peak of the auto-correlation (does not 
need spline) and multiplied by the sampling interval to calculate the time shift at each window, $\tau_{\mathbf{i}}$.

The local displacement at each window, $\mathrm{d}_{i}$, can be calculated from the time shift at each window as follows [31]:

$$
d_{i}=\frac{1}{2}\left(c * \tau_{i}\right)
$$

where $\mathrm{c}$ is the measured ultrasound velocity from Chapter 3, section 3.3.1 Displacement is divided by 2 because the sound wave propagates through the phantom and returns. The strain, $\varepsilon$, or the gradient of the displacement, can also be calculated from the time shifts of the pre- and post-compression signals at each window [32]:

$$
\varepsilon=\frac{\tau_{i+1}-\tau_{i}}{T_{i}}
$$

where $\tau_{\mathrm{i}}$ is the time shift at each window, $\tau_{\mathrm{i}+1}$ is the time shift at the next window and $T_{\mathrm{i}}$ is the time between windows. 


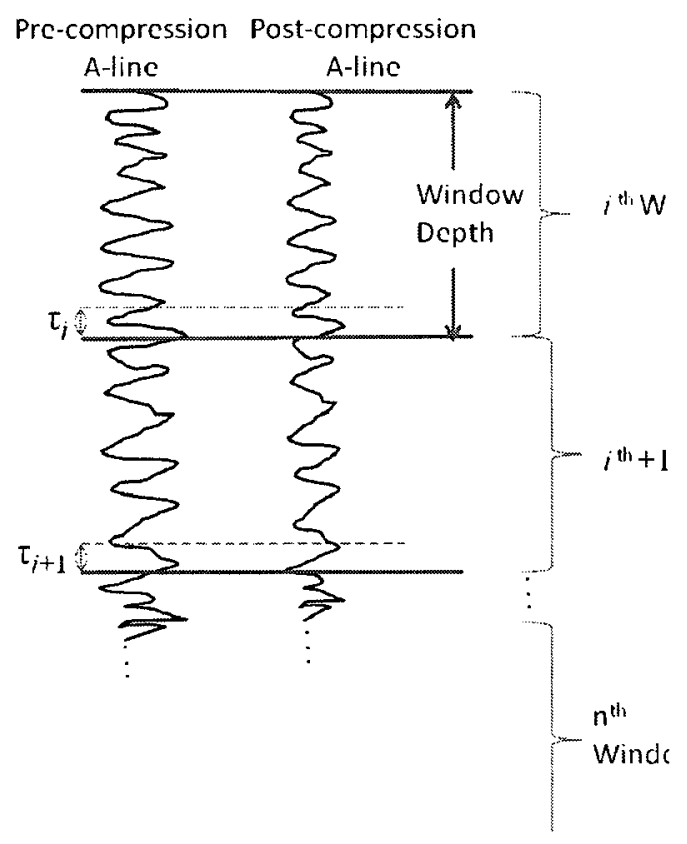

Figure 4.8: Diagram of pre and post-compression RF signals subdivided into windows.

Axial resolution is inversely proportional to the ultrasound system wavelength [33], and thus reducing window lengths and overlapping the windows, as shown in Fig. 4.9, improves axial resolution [34]. Since a center frequency, $f_{c}$, of $10 \mathrm{MHz}$ and an ultrasound velocity of $1490 \mathrm{~m} / \mathrm{s}$ was used from Chapter 3, section 3.3.1, the signal wavelength was calculated as $0.149 \mathrm{~mm}$ using:

$$
\lambda=\frac{c}{f_{\mathfrak{c}}}
$$

Since the system sampled at $0.03 \mu$ s and assume 90 data points were used, then the window length would be $2 \mathrm{~mm}$, or 13 wavelengths $(2 \mathrm{~mm} / 0.149 \mathrm{~mm})$, using:

$$
d_{w}=\frac{1}{2}(c * \text { sampling rate } * \text { data points })
$$




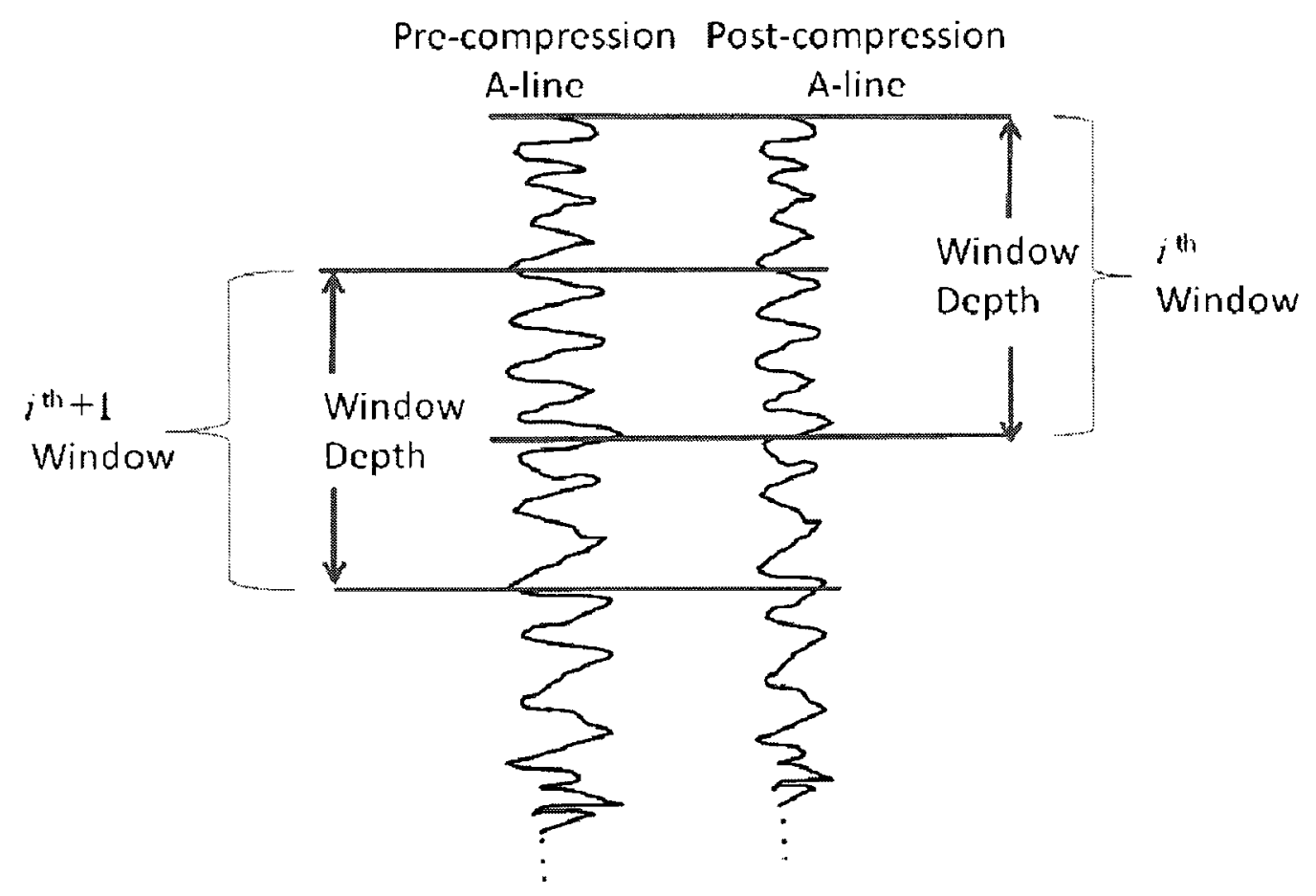

Figure 4.9: Diagram to illustrate overlapping windows to improve axial resolution.

A 2D displacement and strain image can be created using a surface plot of each window for each scanline, across the lateral width of the phantom. The three main types of noise that degrade elastographic quality are sampling noise, decorrelation noise and random noise. Performing spline interpolation not only improves time shift accuracy, but also reduces sampling artefacts [14]. False peaks, one type of sampling artefact, appear as spikes in the displacement and strain images [16]. A reduction in false peaks can also be accomplished with median filtering [33], [14]. A median filter replaces the center pixel in a neighborhood with the median value of its neighbors. For example, a $2 \times 2$ neighborhood would use a $2 \times 2$ matrix area around the center to determine the median value of the 
center. Median filtering helps to remove outliers, or for this thesis, artefact noise. There is a trade-off because increasing the size of the neighborhoods reduces the overall image size. Decorrelation, from tissue compression and scatterer movement noise is seen more with increasing strain [35]. The scatter movement towards the probe shortens the signal in the time domain, and therefore changes the envelope of the signal in the time domain. A shortening of the signal correlates with a widening in the frequency spectrum [35].

A multicompression technique was investigated to reduce signal decorrelation noise. This method uses small compression steps that replace a large compression and then the displacement or strain images are accumulated [35]. The small compression steps were not accomplished through physically compressing the phantoms with increasing strain, but with signal analysis. Without using multicompression the pre- and post-compression frames (total of 2) are used to determine strain. With multicompression the signals were analyzed using the pre- and post-compression frames and all the frames between the pre- and post-compression frames (for $0.8 \mathrm{~mm}$ compression this was 33 frames). The strain between each chronological frame was determined, and then these incremental strains were accumulated. Random noise was not addressed in this thesis.

Signal-to-noise ratio (SNR) is one measurement used to determine image quality, and is calculated in this thesis using displacement or strain [36]:

$$
S N R=\frac{A_{m}}{A_{s t d}}
$$

where $A_{m}$ and $A_{s t d}$ are the mean and standard deviation of the signal amplitude. Contrast, a measure of tumor detectability, as discussed in Chapter 3, section 3.32, is another 58 
method used in this thesis to determine image quality. Contrast can also be measured using strain of the tumor, $\varepsilon_{\mathrm{t}}$, and background, $\varepsilon_{\mathrm{b}}$ :

$$
C_{z}=\frac{\left.\varepsilon_{t}-\varepsilon_{\tilde{g}}\right]}{\varepsilon_{z}}
$$

\subsection{Experimental Results and Discussion}

\subsubsection{Displacement and Strain imaging}

Since the primary goal of this research work was strain imaging, only the dispiacement results that directly contributed to improvement in strain imaging are presented in this thesis. Fig. 4.10 is shown to illustrate poor probe alignment with the pinantom, which resulted in nneven displacement along the scanlines, or along the width of the phantom.

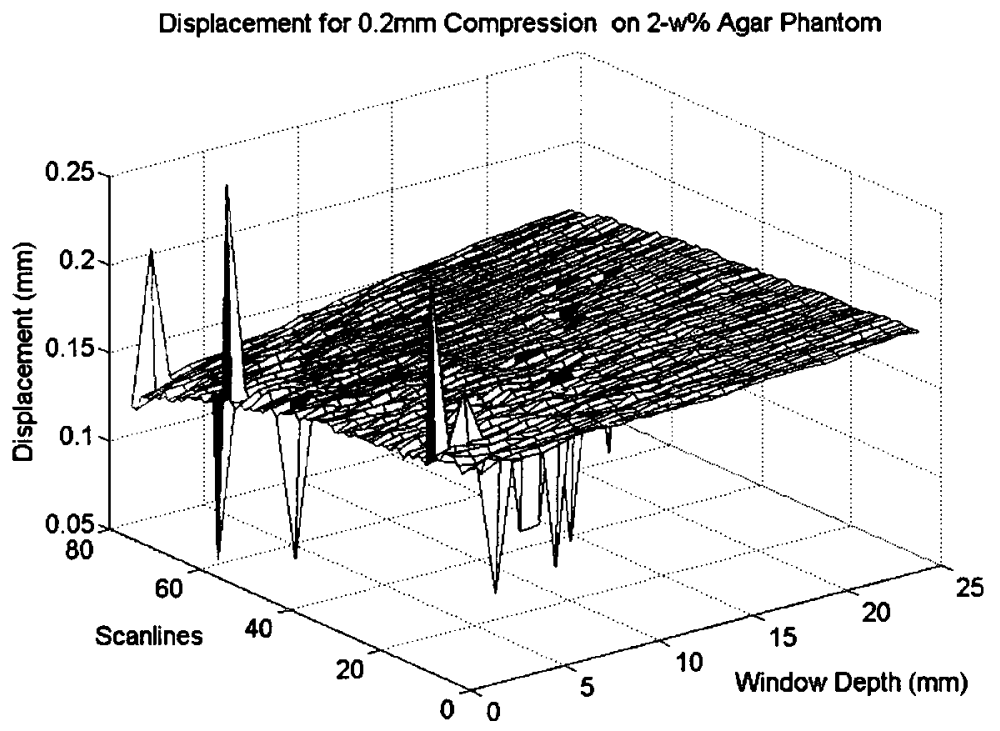

Figure 4.10: Displacement with poor probe alignment. 
The displacement SNR with respect to the window length of the cross-correlation was determined for the $0.6 \mathrm{~mm}$ compression on the 2-w\% agar phantom, as shown in Fig. 4.11. As the window length decreased noise was introduced into the signal, because too few wavelengths were used in the correlation. Improving the SNR was important to improve image quality, but increasing the window length also reduced spatial resolution. It was determined from Fig. 4.11 that the optimum window length was $2 \mathrm{~mm}$ and using a window length greater than $2 \mathrm{~mm}$ did not improve SNR. A window length of $2 \mathrm{~mm}$ was used for all signal processing presented in this thesis.

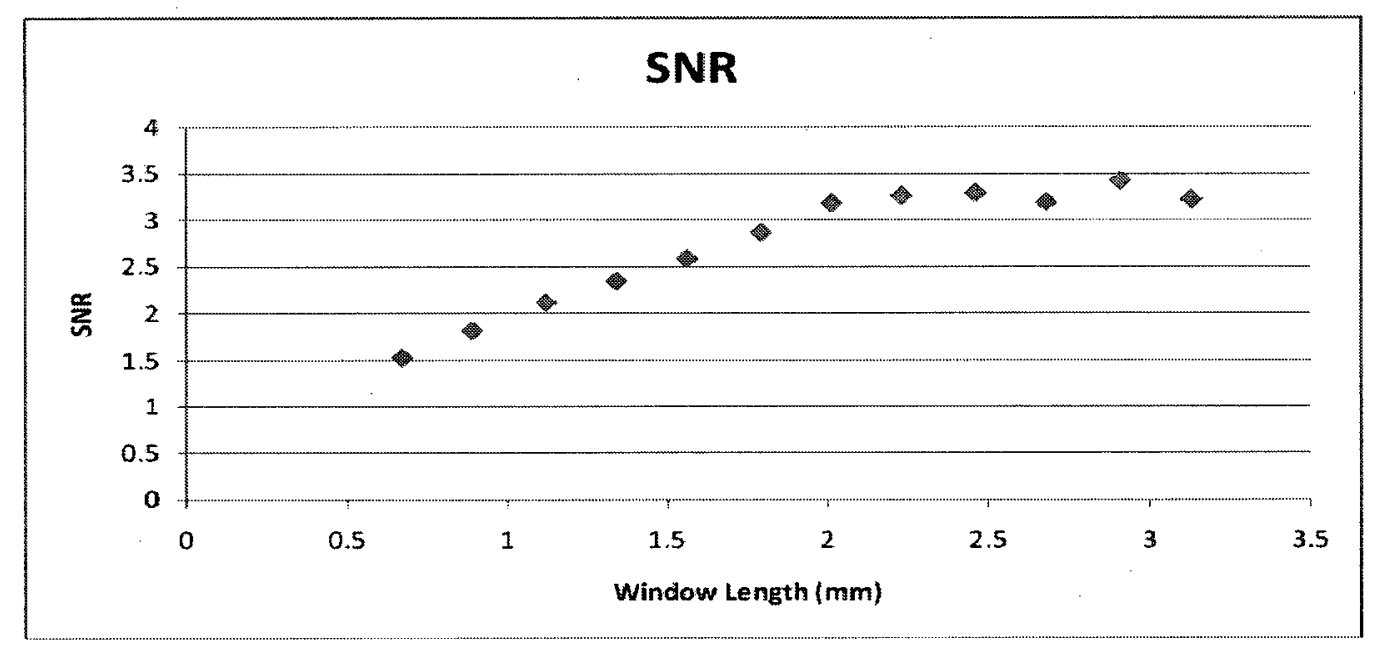

Figure 4.11: Displacement SNR versus increasing window length.

Fig. 4.12 shows tumor strain images for $0.2 \mathrm{~mm}$ compression (a) before filtering and after 2D median filtering using a (b) $2 \times 2$ and (c) $3 \times 3$ neighborhood. Fig. 4.12 (a), (b) and (c) maintains the same strain scale. The same images are shown in 2D in Fig. 4.13 (a), (b) and (c) with the strain scale expressed using a colorbar. Since the window size 
was $2 \mathrm{~mm}$ and overlap 50\%, each pixel represents $1 \mathrm{~mm}$. The full $30 \mathrm{~mm}$ phantom window depth is not shown because of limitations when using overlap and the number of data points. For example, if the window length remained at $2 \mathrm{~mm}$ (or 90 data points) and the overlap was removed (ignore the lens data points), then the image could have 15 windows (each window is $2 \mathrm{~mm}$ ) if there were at least 1350 data points. There would need to be at least 1350 data points before the plexiglas $(90$ data points $\mathrm{x} 15$ windows $=$ 1350 ), since the sample rate was $0.03 \mu$ s, and phantom ultrasound velocity was $1490 \mathrm{~m} / \mathrm{s}$. 
(a)

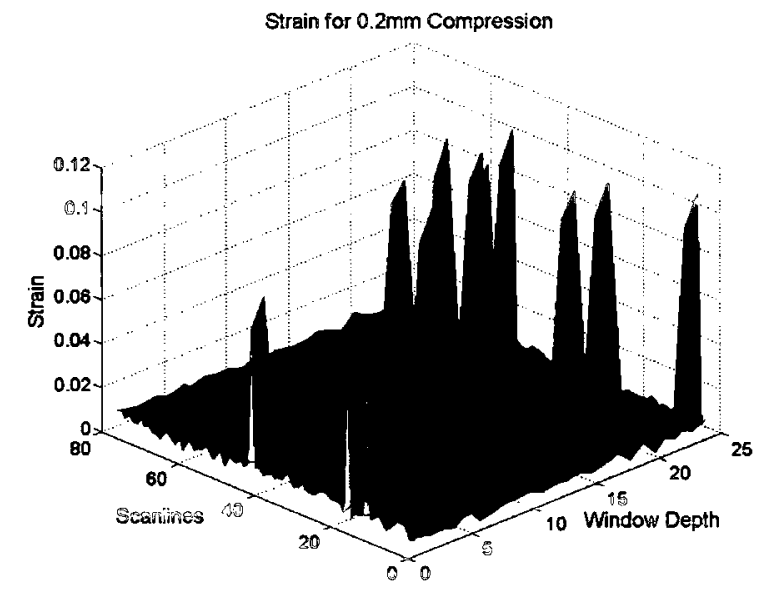

(0)

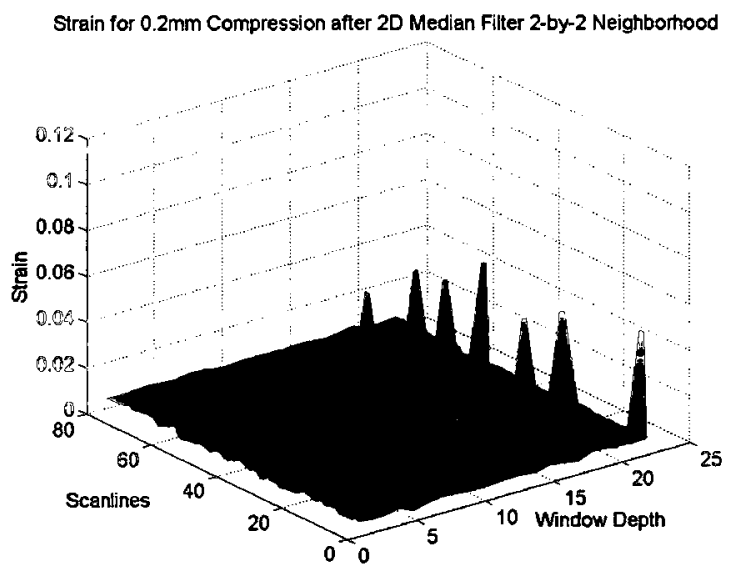

(c)

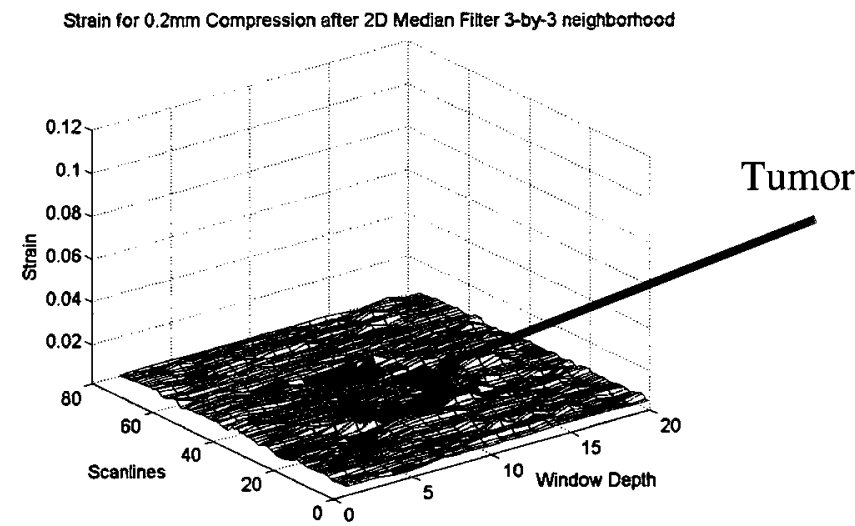

Figure 4.12: Strain images for $0.2 \mathrm{~mm}$ compression (a) before filtering and after median filtering using a (b) $2 \times 2$ and (c) $3 \times 3$ neighborhood. 
(a)
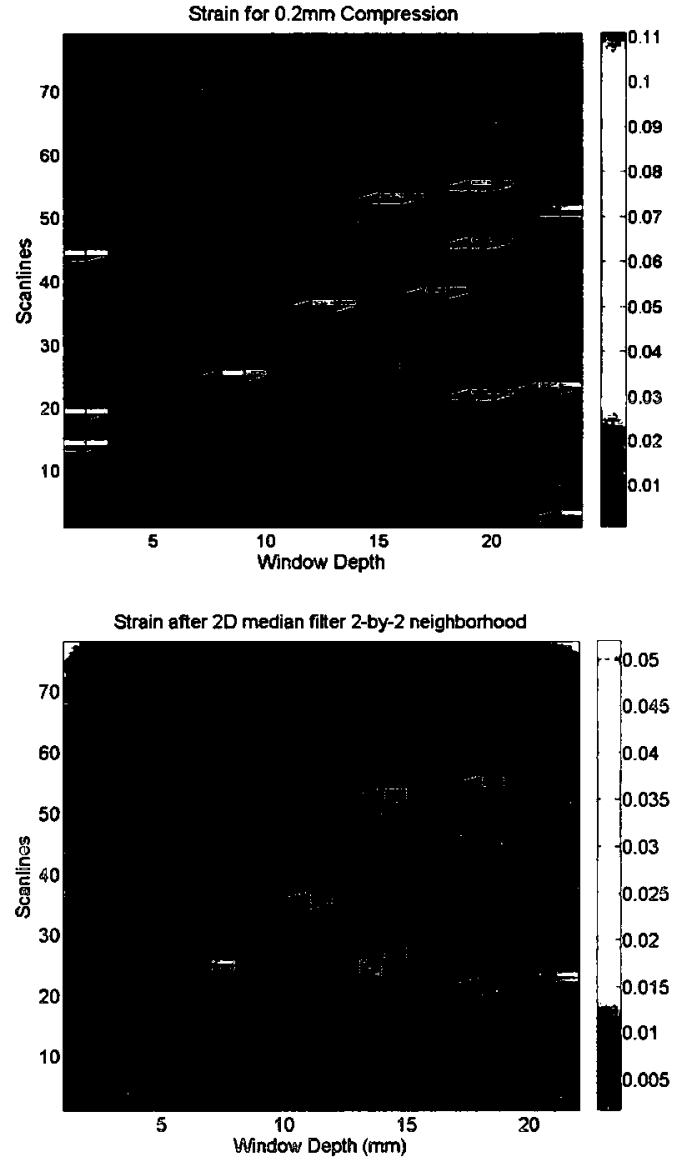

(c)

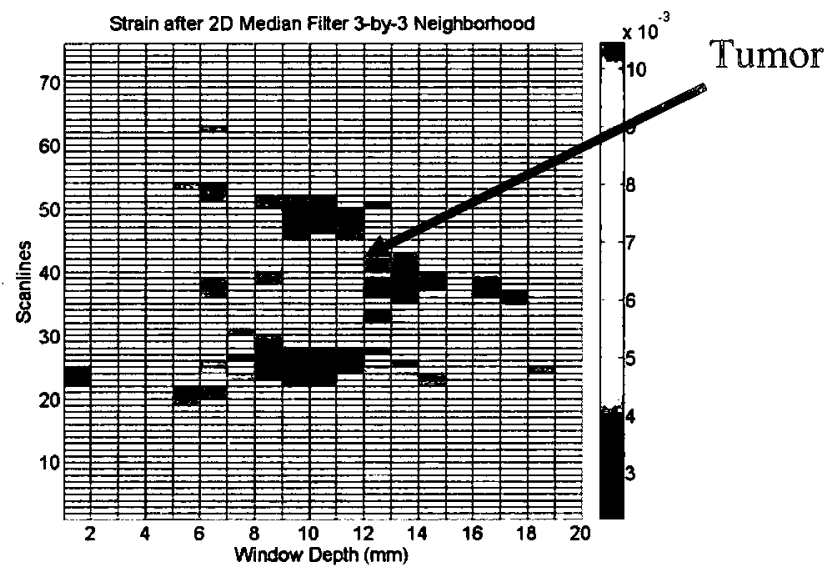

Figure 4.13: Strain images for $0.2 \mathrm{~mm}$ compression (a) before filter and after median filtering using a (b) $2 \times 2$ and ( $3 \times 3)$ neighborhood. Colorbar indicates strain. 
Fig. 4.14 shows tumor strain images for $0.8 \mathrm{~mm}$ compression without multicompression (a) before filtering and after 2D median filtering using a (b) $2 \times 2$ and (c) $4 \times 4$ neighborhood. The expected strain for $0.8 \mathrm{~mm}$ compression on a phantom with $30 \mathrm{~mm}$ depth is 0.027 . Fig. 4.15 shows tumor strain images for $0.8 \mathrm{~mm}$ compression with multicompression (a) before filtering and after a 2D median filter using a (b) $2 \times 2$ and (c) $3 \times 3$ neighborhood. 

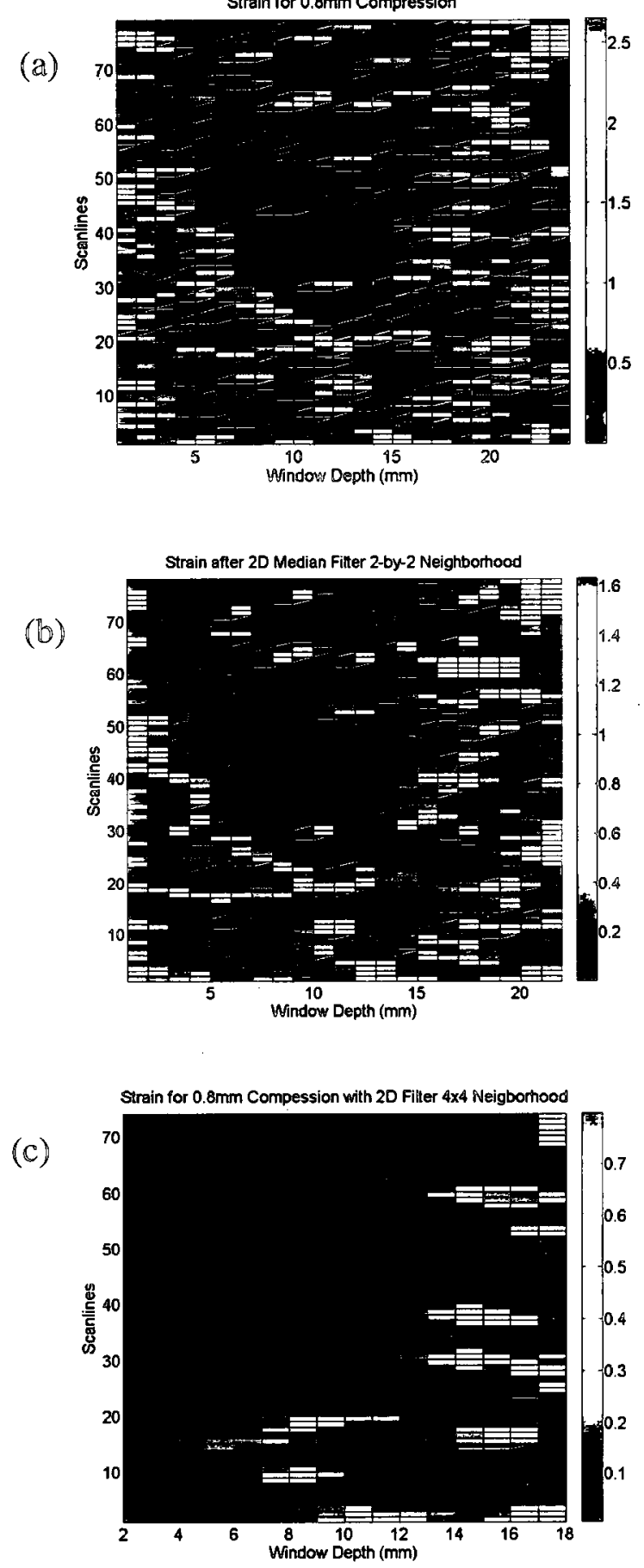

Figure 4.14: Strain image for $0.8 \mathrm{~mm}$ compression (a) before filter and after median filtering using a (b) $2 \times 2$ and (c) $3 \times 3$ neighborhood. 
(a)

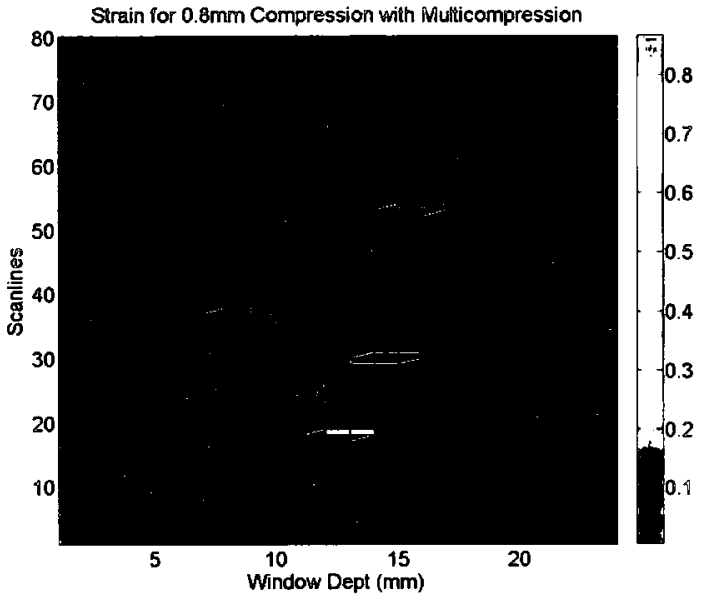

(b) Strain for $0.8 \mathrm{~mm}$ Compression with Multicompression and 2D Filter

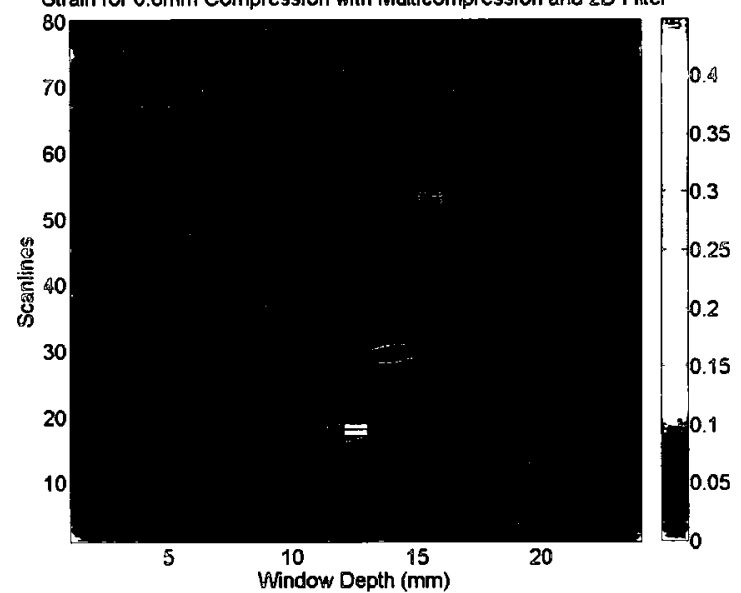

(C) Strain for $8 \mathrm{~mm}$ Compressoion with Multicompression and 20 Filter $3 \times 3$ Neighborhood

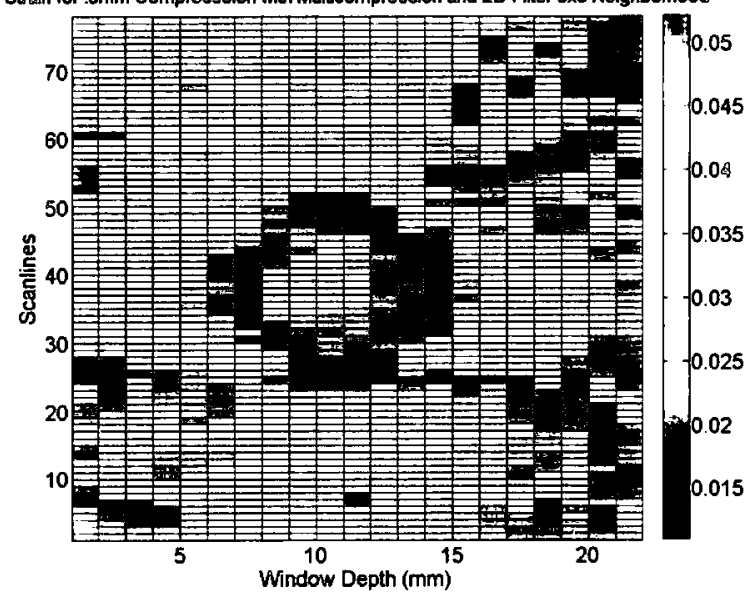

Figure 4.15: Strain image for $0.8 \mathrm{~mm}$ compression with multicompression (a) before filtering and after filtering using a (b) $2 \times 2$ and (c) $3 \times 3$ neighborhood. 
Qualitatively the images after multicompression for $0.8 \mathrm{~mm}$ compression in Fig. 4.15 (a) through (c) have improved delineation of the tumor boundaries compared to the images created without multicompression in Fig. 4.14 (a) through (c). The $0.8 \mathrm{~mm}$ compression images with multicompression, shown in Fig. 4.15 (a) through (c), appear more similar to the $0.2 \mathrm{~mm}$ compression images, as shown in Fig. 4.13 (a) through (c). The preferred images (qualitative) to visually observe the tumor are with $0.2 \mathrm{~mm}$ compression after 2D median filtering with a $2 \times 2$ neighborhood and $0.8 \mathrm{~mm}$ compression after multicompression and a 2D median filter with a 2x2 neighborhood, as shown in Fig. 4.13 (b) and Fig. 4.15 (b), respectively.

The tumor and background strain SNRs were calculated for strain images without and with multicompression and before and after filtering. The tumor and background SNRs were calculated using eqn. 4.5 and strain measurements (mean and standard deviation) from within the marked areas, as shown in Fig. 4.16. Note that the background area includes the area enclosed with dotted lines. The dotted lines were used to select a different background area to calculate contrast. In addition, the average of the tumor and background strain SNR was calculated to provide an overall image quality. The tumor, background and overall strain mean and SNR without and with multicompression are summarized in Tables 1 and 2, respectively. The background mean strain was also calculated for the areas enclosed within the dotted lines.

In addition to SNR, image contrast was also calculated for images without and with multicompression and before and after filtering. Contrast was calculated using eqn. 
4.6, from Chapter 4, section 4.1.2, and tumor and background mean strains. Image contrast results with and without multicompression are presented in Tables 1 and 2, respectively. Contrast calculation was repeated using eqn. 4.6 , from Chapter 4 , section 4.1.2, and the means of the tumor and the background areas enclosed within the dotted lines (a different background area). This different background area was chosen to demonstrate how the selection of areas for contrast calculations can affect results.

Young's modulus of the tumor and background were calculated using the maximum balance stress measurements and the ultrasound mean tumor and background strains and eqn. 3.3, from Chapter 3, section 3.2.3. The background mean strains were calculated using the areas enclosed within the dotted lines. 
Strain for .8mm Compressoion with Multi-compression and 2D Filter $3 \times 3$ Neighborhood

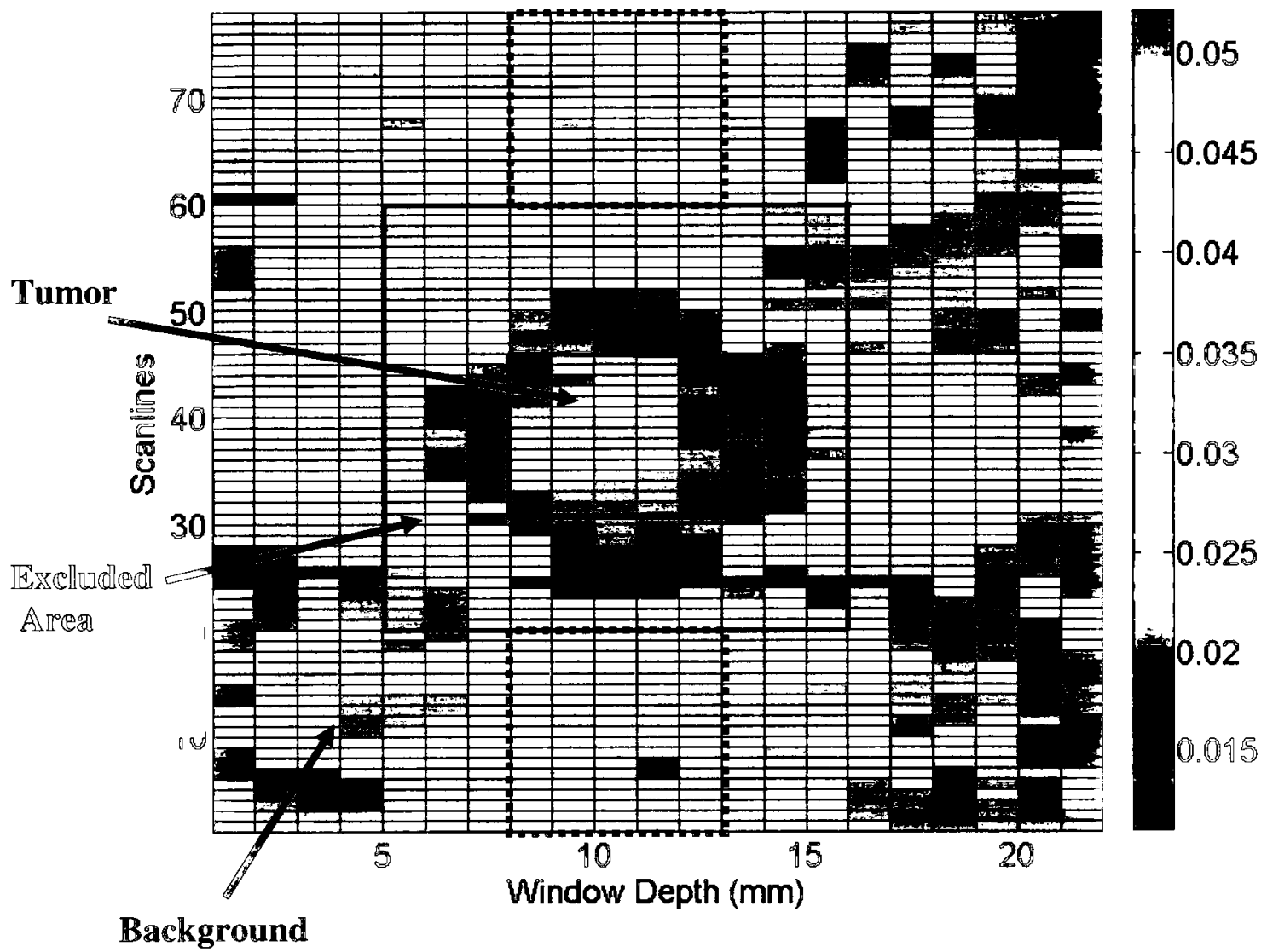

Figure 4.16: Strain image with marked areas used for $\mathbb{S N R}$ and comtrast calculations. 
Table 1: Results for $0.8 \mathrm{~mm}$ Compression without Multicompression.

\begin{tabular}{|l|l|l|l|l|l|l|}
\hline $\begin{array}{c}\text { 2D } \\
\text { Median } \\
\text { Filter }\end{array}$ & $\begin{array}{c}\text { Tumor } \\
\text { Mean } \\
\text { Strain }\end{array}$ & $\begin{array}{c}\text { Tumor } \\
\text { SNR }\end{array}$ & $\begin{array}{c}\text { Backgound } \\
\text { Mean } \\
\text { Strain }\end{array}$ & $\begin{array}{c}\text { Backgound } \\
\text { SNR }\end{array}$ & $\begin{array}{c}\text { Average } \\
\text { SNR }\end{array}$ & Contrast \\
\hline None & 0.0277 & 0.88 & 0.1649 & 0.77 & 0.83 & 0.832 \\
\hline $\begin{array}{l}\text { 2D Filter } \\
\text { (2x2) }\end{array}$ & 0.0236 & 2.22 & 0.1256 & 1.03 & 1.62 & 0.8119 \\
\hline $\begin{array}{l}\text { 2D Filter } \\
(3 \times 3)\end{array}$ & 0.0201 & 13.54 & 0.0848 & 1.04 & 7.29 & 0.7634 \\
\hline
\end{tabular}

Table 2. Results for $0.8 \mathrm{~mm}$ Compression with Multicompression.

\begin{tabular}{|c|c|c|c|c|c|c|}
\hline $\begin{array}{c}\text { 2D } \\
\text { Median } \\
\text { Filter }\end{array}$ & $\begin{array}{c}\text { Tumor } \\
\text { Mean } \\
\text { Strain }\end{array}$ & $\begin{array}{c}\text { Tumor } \\
\text { SNR }\end{array}$ & $\begin{array}{c}\text { Backgound } \\
\text { Mean Strain }\end{array}$ & $\begin{array}{c}\text { Backgound } \\
\text { SNR }\end{array}$ & $\begin{array}{c}\text { Average } \\
\text { SNR }\end{array}$ & Contrast \\
\hline None & 0.0252 & 1.44 & 0.025 & 3.63 & 2.53 & 0.0093 \\
\hline $\begin{array}{c}\text { 2D Filter } \\
(\mathbf{2 x 2})\end{array}$ & 0.0236 & 3.65 & 0.0236 & 5.06 & 4.36 & 0.0094 \\
\hline $\begin{array}{c}\text { 2D Filter } \\
(\mathbf{3 x 3})\end{array}$ & 0.0214 & 16.75 & 0.0232 & 7.67 & 12.2 & 0.0758 \\
\hline
\end{tabular}

The background SNR increased with multicompression, as shown in Fig. 4.18. Since the background SNR increased with multicompression, because of less decorrelation noise, contrast was reduced (less signal variation in the background means less image contrast). The background mean strain was also more accurate, closer to the expected strain of 0.027 , with multicompression, as shown in Table 2. Although not presented in Table 2, the background mean strain was 0.0267 when the background areas enclosed within the dotted lines, shown in Fig. 4.16, were used.

The median filter had a significant affect on image contrast, as shown in Fig. 4.19. In addition, contrast improved significantly, from 0.0758 to 0.19 , when calculated using the background from the areas enclosed within the dotted lines instead of the larger 
background area, as shown in Fig. 4.16. These different contrast results indicate that the areas selected for contrast calculations is crucial.

After the 2D median filter was applied to reduce artifact noise there was an improvement to image quality, as shown by an increase in tumor and background SNRs with multicompression in Fig. 4.17 and 4.18. The tumor SNR increased using increasing neighborhoods (from $2 \times 2$ to $3 \times 3$ neighborhood) for the median filter without and with multicompression, as shown in Fig. 4.17. The background SNR also increased using increasing neighborhoods for the median filter with multicompression, however the background SNR did not change after increasing the neighborhoods for the median filter without multicompression, as shown in Fig. 4.18.

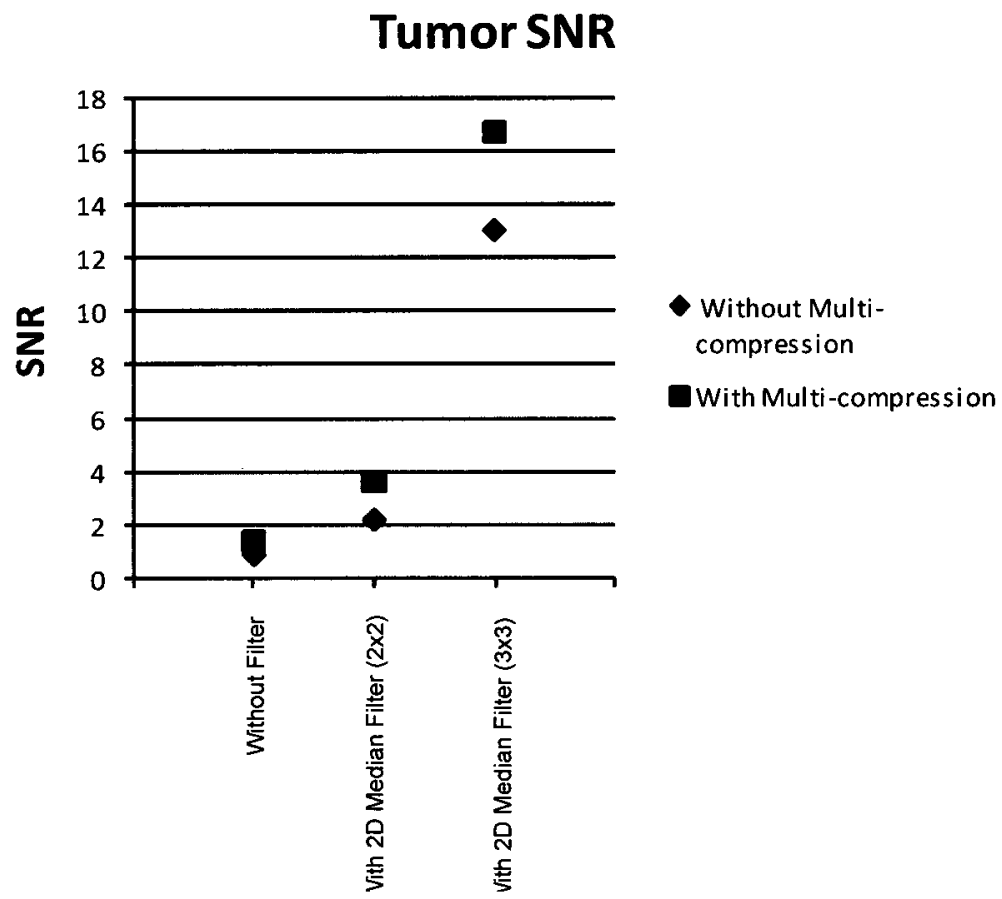

Figure 4.17: Tumor SNR without and with multicompression. 


\section{Background SNR}

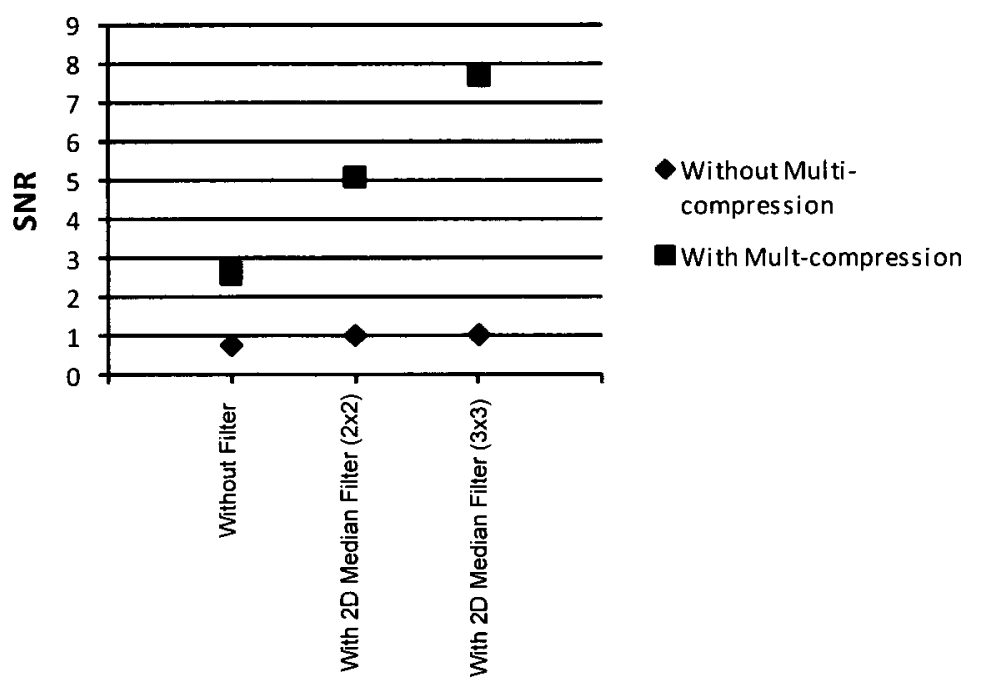

Figure 4.18: Background SNR without and with multicompression.

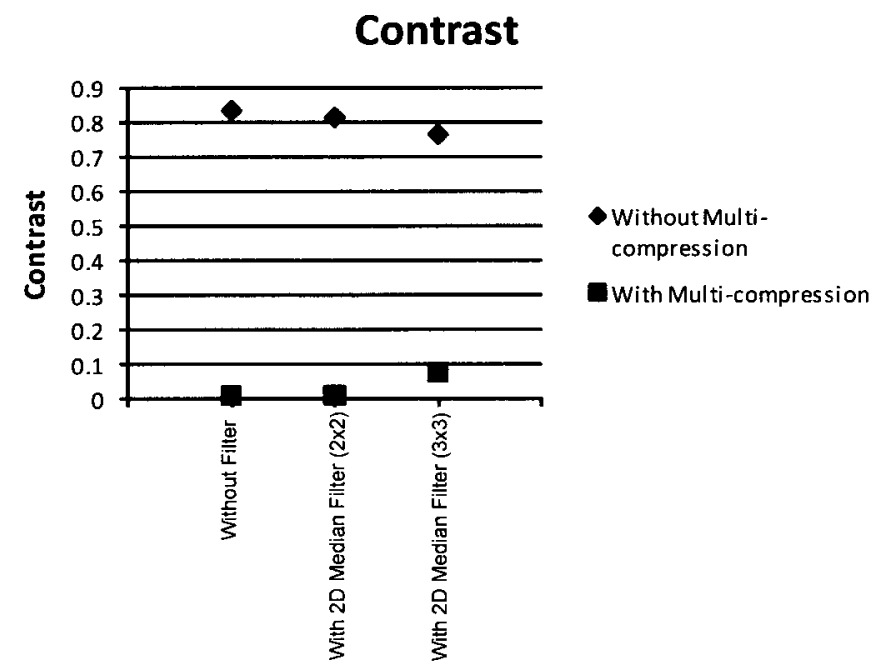

Figure 4.19: Contrast before and after median filtering. 


\subsubsection{Young's Modulus from Ultrasound Strain Images}

Fig. 4.20 shows the tumor and background stress-strain curves using the stress peaks from the strain imaging experiments, as described in section 4.1.2, and the ultrasound mean tumor and background strains for $0.2 \mathrm{~mm}, 0.4 \mathrm{~mm}$ and $0.8 \mathrm{~mm}$ compressions. The tumor mean strains were calculated using the area marked in Fig. 4.16 and the background mean strains were calculated from the areas within the dotted lines in Fig. 4.16. All strains in the stress-strain curves in Fig. 4.20 were calculated after median filtering with a $3 \times 3$ neighborhood. The mean strains measured for $0.2 \mathrm{~mm}$ and $0.4 \mathrm{~mm}$ compressions were analyzed without multicompression, while the $0.8 \mathrm{~mm}$ compression data were analyzed with multicompression to reduce decorrelation noise.

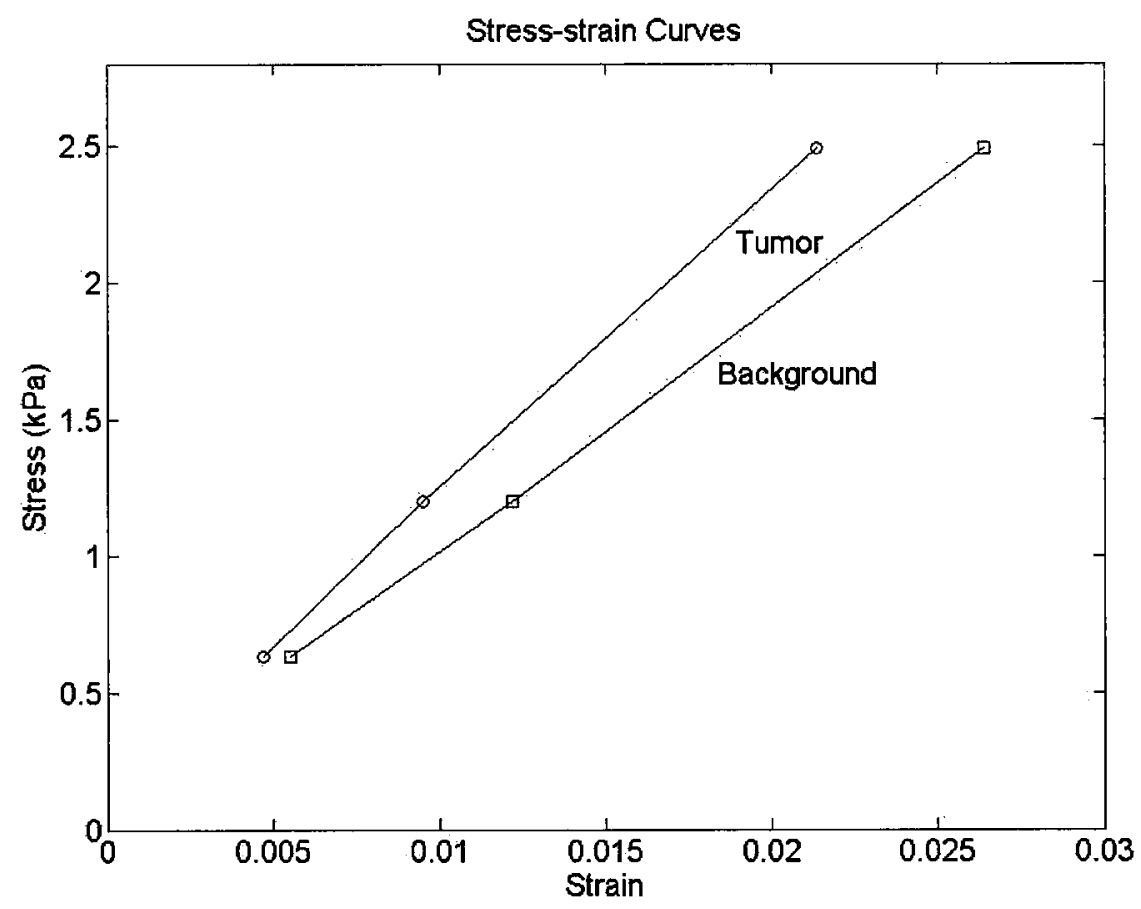

Figure 4.20: Original (markers) and linear fit (solid line) for tumor phantom stress-strain curves. 
Since the slope of the tumor stress-strain curve was greater than the slope of the background stress-strain curve, the tumor is stiffer than the background. The original ultrasound mean tumor and background stress-strain curves and the linear fit for the stress-strain curves are shown in Fig. 4.20. The Young's modulus of the background and tumor were determined to be $89 \mathrm{kPa}$ and $111 \mathrm{kPa}$, respectively. The Young's modulus calculated in chapter 3 , section 3.3 .3 , was $74 \mathrm{kPa}$ and $174 \mathrm{kPa}$ for the $2-\mathrm{w} \%$ and $3-\mathrm{w} \%$ agar phantoms (without ultrasound). One possible reason for the difference in Young's modulus is contact. A preload, which was implemented during tumor ultrasound experiments, provided improved contact between the phantom and the probe. This improved contact from preload ensured that the full compression was applied to the phantom. If poor contact existed between the phantom and the transducer/mechanical arm during stress-relaxation measurements, then the full compression may not have been applied. Less compression results in lower strain than expected, thus Young's modulus could be different. In addition, Young's modulus was determined from measured ultrasound strain in Fig. 2.10, unlike the Young's modulus from Fig. 3.22, which was determined from expected strain. Other possible factors that could explain the difference in Young's modulus are boundary conditions, temperature and age of the phantoms, to name a few. Unlike the single concentration phantoms that were constructed for stressrelaxation measurements, tumor phantoms were constructed with different agar concentrations and boundary conditions between the tumor and the background could have influenced measurements. Changes in temperature will change the Young's 
modulus of the phantoms. As phantoms age they dehydrate, and thus their Young's modulus changes over time.

Young's modulus of a 1.5-w\% and 3-w\% agar phantom was measured as $40 \mathrm{kPa}$ and $90 \mathrm{kPa}$, respectively, using atomic force microscopy (AFM) by a research group in Sapporo, Japan [37]. The Young's modulus measured in this thesis (89 $\mathrm{kPa}$ for 2-w\% and $111 \mathrm{kPa}$ for $3-\mathrm{w} \%$ by ultrasound and $74 \mathrm{kPa}$ for $2-\mathrm{w} \%$ and $174 \mathrm{kPa}$ for $3-\mathrm{w} \%$ by force measurement) was greater than the Young's modulus measured by the research group in Japan, assuming a linear relation between agar concentration and Young's modulus. Assuming a linear relationship between agar concentration and Young's modulus a 2-w\% agar phantom would have a Young's modulus of approximately $57 \mathrm{kPa}$. As previously discussed, there are other factors that impact the Young's modulus of a material, such as age of the phantoms, boundary conditions and temperature. Perhaps, given these influencing factors, the Young's measured compares sufficiently. 


\section{Chapter 5: \\ Conclusions and Future Work}

This chapter concludes this thesis, summarizes findings and suggests future work.

\subsection{Conclusions}

This thesis provided background on ultrasound physics, the methods used to determine soft tissue stiffness, two mechanical properties (stress-relaxation and hysteresis) of soft biological tissues that impact elastic imaging and one acoustic property, ultrasound velocity that was also used to create displacement images. Various agar-based isotropic single concentration phantom construction methods were presented. The constructed phantoms ultrasound velocity, stress-relaxation, Young's modulus and hysteresis were investigated and presented. Methods to construct tumor mimicking phantoms, and to perform displacement and strain imaging were then discussed. The displacement and strain imaging results were presented and discussed.

To conclude this thesis, the methods implemented to create strain images will be discussed. Next, results from the stress-relaxation, Young's modulus and hysteresis are concluded, and then in the last part of this section the ultrasound velocity results are concluded.

To improve image resolution and SNR the appropriate window length for crosscorrelation was selected. It was determined that the window length was $2 \mathrm{~mm}$ and beyond $2 \mathrm{~mm}$ there was little gain in SNR under the experimental conditions employed in this 
thesis. Should a different center frequency, other than $10 \mathrm{MHz}$, be used, then it is recommended that a new optimal window length be determined. Overlapping adjacent windows was also implemented and further improved axial resolution.

Several signal processing methods were also investigated to reduce noise, and thus improve the quality of the strain images for soft tissue mimicking phantoms. A multicompression technique was investigated and found to have reduced decorrelation noise, and thus improved strain SNR and measurement accuracy. In order to improve axial resolution, spline interpolation was employed in the signal processing to determine the phantom displacement and strain. 2D median filtering with two different neighborhoods were performed to remove outlier data points caused by the low correlation regions in the ultrasound data. Spline interpolation increased time shift accuracy and improved overall image quality. The median filter increased tumor and background SNR with multicompression. Increasing the neighborhoods (from $2 \times 2$ to $3 \times 3$ ) improved SNR, but there was a small loss of image at the image edge (one pixel $=1$ $\mathrm{mm}$ around the perimeter of the image).

Although multicompression, spline interpolation, 2D median filtering improved image quality, the quality of the image did not improve with implementation of a Hilbert transform. The Hilbert transform was investigated to take into account the possible phase shift of the post-compression signal, but no change was seen in the tumor and background mean strains and SNR.

It was determined through analyzing the stress-relaxation curves that the phantoms relaxed under constant strain and that contrast existed for 2-w\% and 3-w\% 
phantoms. Because the phantoms relax under constant strain, the Young's modulus of the phantom depends on when the stress was measured.

Using the peak stress measurements from the stress-relaxation data and the known applied strain, the stress-strains curves for the 2-w\% and 3-w\% agar phantoms were created and from analyzing the slope of the curves it was found that the 3-w\% agar phantom was stiffer than the 2-w\% agar phantom. In addition, linear fitting of the slopes of the stress-strain curves gave a Young's modulus of $74 \mathrm{kPa}$ and $174 \mathrm{kPa}$ for 2-w\% and 3-w\% phantoms, respectively.

Using the peak stress measurements from the ultrasound stress imaging experiments and the ultrasound tumor and background mean strain, the stress-strains curves for the background and the tumor were created and from analyzing the slope of the curves it was found that the tumor was stiffer than the background. In addition, linear fitting of the slopes of the stress-strain curves gave a Young's modulus of $89 \mathrm{kPa}$ and 111 $\mathrm{kPa}$ for the background and tumor, respectively. Since the Young's modulus is dependent on experimental conditions (phantom material additives, pre-load conditions and strain rate) it can be difficult to compare these results to other Young's modulus results in literature. A preload was applied for ultrasound tumor phantom experiments, but was not used for the stress-relaxation experiments.

The 2-w\% agar phantom experienced hysteresis and thus the stress-strain curves and Young's modulus are also dependent on whether the phantoms are being loaded or unloaded during data collection. 
In broadband reflection substitution technique to measure the ultrasound velocity of soft tissue mimicking phantoms, a method to place a thin $\mathrm{Al}$ foil reflector at the water/phantom interface has been proposed in order to improve the SNR of the ultrasound echo reflected from the phantom sample. According to the numerical calculation conducted to investigate the effect of the $\mathrm{Al}$ foil thickness on the velocity measurement, the error was estimated $0.05 \%$ for the $\mathrm{Al}$ foil having thickness of $15 \mu \mathrm{m}$ under the measurement conditions employed. If the required accuracy of the velocity measurement is around $0.1 \%$, the proposed technique would be a simple and convenient way to improve the SNR of the desired echo.

\subsection{Challenges}

There were many challenges to overcome in this thesis research. Two obstacles associated with elastic imaging were probe misalignment and poor contact for imaging. The misalignment from positioning the probe slightly off normal with the phantom surface gave undesired results. Methods that improved the slicing of the phantom into parallel sides included the design and construction of a more accurate slicing device. In addition, alignment was overcome through careful levelling of the probe with the mechanical arm, levelling the mechanical arm with the Plexiglas at the base of the Pyrex container (which housed the phantom during experiments) and the balance. Using a preload helped to achieve good contact between the probe and the phantom. Ultrasound realtime B-mode images of the phantom and the Plexiglas reflector attached at the bottom surface of the phantom helped to achieve good alignment and contact. 
Since all experiments were performed with phantoms their limitations would need to be discovered and overcome. When the phantoms were tested in dry air they became dehydrated and the weight of the phantom changed, albeit slightly, from the start to the end of the experiment. This was overcome by testing the phantoms in water, which kept the phantoms hydrated. Since the phantoms were being tested in water the weight of the water displacement from the mechanical arm and probe would need to be accounted for, so a procedure to determine the weight of the mechanical arm and the probe in the water with each compression was implemented. It was also determined that storing the phantom in silicone molds at room temperature in a humid environment, rather than wrapped in plastic in the fridge, also helped to maintain the phantoms. The phantom edges were no longer misshaped from the plastic wrap, but more importantly the phantoms did not get dehydrated and lasted longer.

\subsection{Future Work}

\section{Image Quality:}

Although SNR, axial resolution and contrast were investigated to evaluate image quality, other parameters that evaluate image quality could also be investigated. Lateral resolution, contrast-to-noise (CNR) and sensitivity are other commonly used parameters to provide insight into image quality. Lateral resolution is a measure of the separation between two adjacent points in an image in the transverse plane [1], [38]. Contrast takes into account the estimated backgrounds and tumors mean strains, while CNR takes into account the means and the standard deviations of the estimated tumor and background strains. Although these measurements were collected (used for SNR) the CNR was not 
presented in this thesis, and is thus recommended as future work. Sensitivity is the lowest measurable strain possible and gives insights into the limitations of the system. Matlab simulations would also prove worthwhile to investigate the various parameters used to evaluate images [35].

Investigating other image processing methods to improve image quality would be recommended. Random noise was not addressed in this thesis, but if random noise were to be addressed then one approach could be averaging ultrasound strain images [35]. It is also recommended that other methods used to further reduce decorrelation noise be investigated. One method is temporal stretching alone or in combination with multicompression. Temporal stretching was shown to further reduce axial decorrelation and thus increase SNR after multicompression [35]. Temporal stretching, changes the peak locations of the post-compression RF signal to match the peaks locations of the precompression RF signal [35]. There are also many ways of performing a Hilbert transform and one method not investigated in this thesis was using a FIR filter [16]. One other recommendation to improve image quality is multicompression with spectral crosscorrelation methods [35]. Using multicompression would reduce the phase shift uncertainties, which are present when axial motion is greater than a quarter of an ultrasonic wavelength.

\section{Other Phantom Materials:}

Investigating other phantom materials, such as gelatine which is commonly used for strain imaging, and using materials with a known stiffness for the tumor centers would benefit future research endeavours. To investigate tumor/background boundary 
conditions a tumor phantom could be constructed with 2-w\% agar (instead of 3-w\% agar) for the tumor and background. Other materials to consider are those which form tumor boundaries, since benign tumors are often encapsulated and not invading surrounding tissue. Investigating the properties of benign and malignant tumors and creating phantoms that resemble both benign and malignant tumors would be another recommendation. There are also tumor classification methods that use various strain features to differentiate if a tumor is benign or malignant [39].

\section{Ultrasound Velocity:}

In future studies, a thinner $\mathrm{Al}$ foil less than $15 \mu \mathrm{m}$ will be tested to reduce the measurement error. In addition, a more complete measurement model will be investigated by considering multiple interference echoes reflected from the $\mathrm{Al}$ foil. Other factors that may improve measurement variability could be considered. For phantom development and fabrication, additives such as glycerol (ultrasound velocity $1760 \mathrm{~m} / \mathrm{s}$ ) could be added to agar-based phantoms to increase and/or adjust the ultrasound velocity to a desired value [40].

\section{Hysteresis:}

Future work to determine phantom hysteresis could focus on using software to create a synchronized signal that would indicate the start and finish of the compression load and unload on the weight recordings. Also, a pre-compression prior to the first load cycle (pre-load) is also recommended to ensure good contact between the mechanical arm and the phantom. 


\section{Appendix A}

Various agar concentrations phantoms were constructed for characterization and elastic imaging. The method to construct the phantoms is outlined below and shown in the flowchart in

Fig. A.1. To construct uniform, single concentration phantoms follow steps 1-4, and then (a) through $(\mathrm{c})$. To construct tumor phantoms follow steps $1-4$, and then $\left(\mathrm{a}^{*}\right)$ through $\left(\mathrm{g}^{*}\right)$.

Phantom construction method:

1. Add agar ( $2 \mathrm{~g}$ for $2-\mathrm{w} \%$ agar) to $100 \mathrm{ml}$ degassed distilled water.

2. Heat and stir (alternate) to dissolve and combine the agar.

3. Stir to cool

4. Add carbon graphite powder.

for uniform phantom:

a) Pour into uniform silicone mold.

b) Place into ice water bath until set.

c) Store molded phantom in covered container partially filled with water.

for tumor phantom:

$\left.a^{*}\right)$ Pour into phantom silicone mold.

$\left.b^{*}\right)$ Place molded phantom into ice water bath until set.

c*) Repeat steps 1-4 using desired amount of agar for phantom center (tumor).

$\mathrm{d}^{*}$ ) Unmold phantom \& place into uniform mold.

$\left.\mathrm{e}^{*}\right)$ Tilt molded phantom and pour mixture into phantom hole.

$f^{*}$ ) Place molded phantom into ice water bath until set.

$\left.\mathrm{g}^{*}\right)$ Store molded phantom in covered container partially filled with water. 


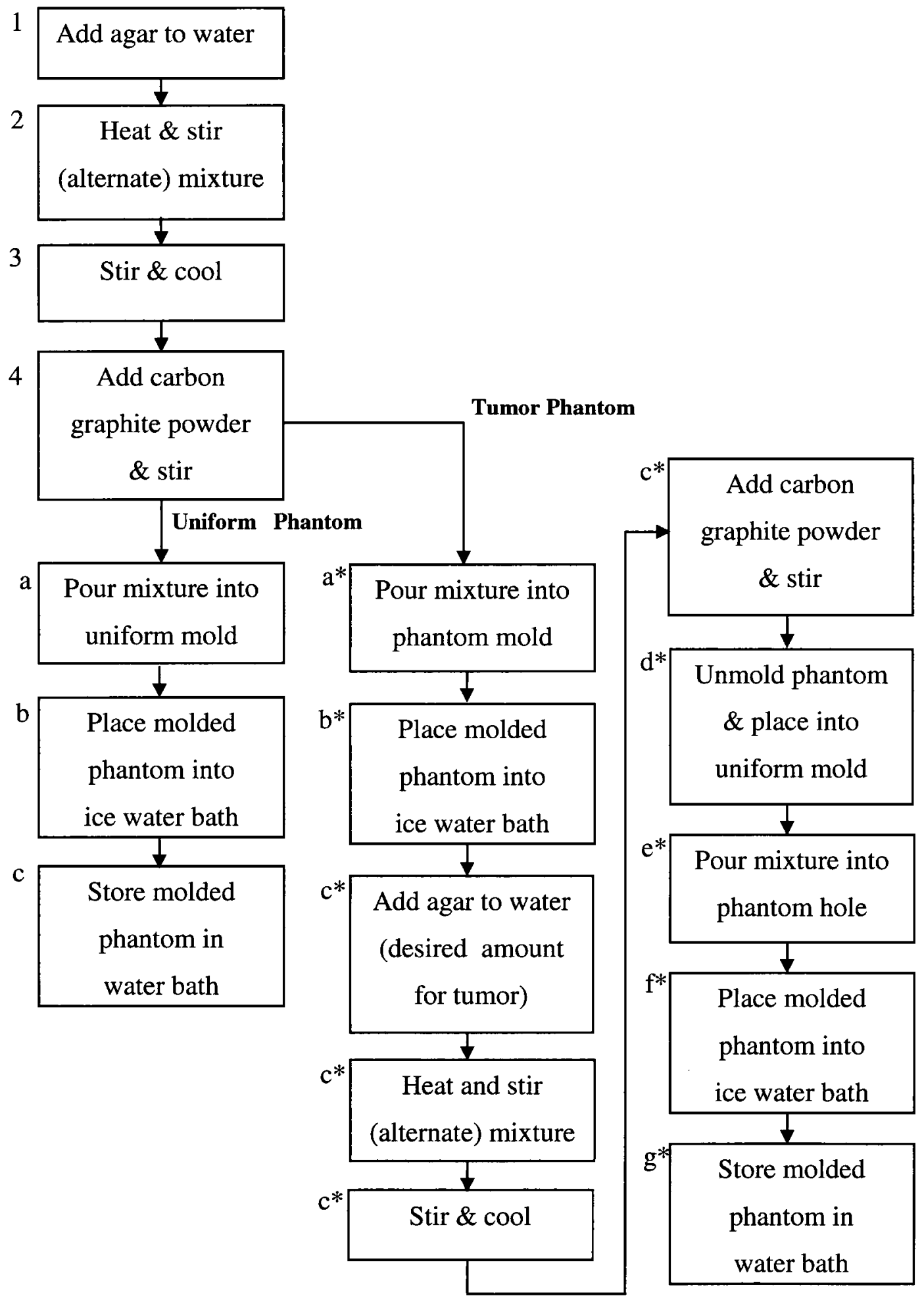

Figure A.1: Flowchart of phantom construction. 


\section{References}

[1] J. L. Prince and J.M. Links, Medical Imaging Signals and Systems, Upper Saddle River, NJ: Pearson Prentice Hall, 2006.

[2] P.R. Hoskins, A. Thrush, K. Martin and T.A. Whittingham, Diagnostic Ultrasound Physics and Equipment, London, England: Greenwich Medical Media Limited, 2003.

[3] W. R. Hedrick, D. L. Hykes, and D. E. Starchman, Ultrasound Physics and Instrumentation, $4^{\text {th }}$ ed., Missouri, U.S.A: Elesvier Mosby, 2005.

[4] J. Greenleaf, "Selected Methods for Imaging Elastic Properties of Biological Tissues," Annual Review Biomedical Engineering, vol. 5, pp. 57-78, 2003.

[5] F. W. Kremkau, Diagnostic Ultrasound: Principles and Instruments, $7^{\text {th }}$ ed., Missouri, U.S.A: Saunders Elesvier, 2006.

[6] T. L. Szabo, Diagnostic Ultrasound Imaging, Burlington, MA: Elsevier Academic Press, 2004.

[7] J. Browne, K. Ramnarine, A. Watson, and P. Hoskins, "Assessment of the acoustic properties of common tissue-mimicking test phantoms," Ultrasound in Medicine and Biology, vol. 29, no. 7, pp. 1053-1060, 2003.

[8] E. Madsen, J. Zagzebski, R. Banjavie, and R. Jutila, "Tissue mimicking materials for ultrasound phantoms," Am. Assoc. Phys. Med., vol. 5, no. 5, pp. 391-394, 1978.

[9] J., Ophir, "Elastography: Imaging the Elastic Properties of Soft Tissues with Ultrasound," Journal Medical Electronics, vol. 29, pp. 155-171, 2002.

[10] R. Souchon, Physics for Medical Imaging Applications, Dordrecht, The Netherlands: Springer Netherlands, 2007.

[11] U. Generoso, "Ultrasound Elastography: A Possible Improvement to the Paraphernalia of Pancreatic Imaging," Journal of Pancreas, vol. 9, pp. 666-667, 2008. 
[12] J. Ophir, B. Garra, F. Kallel, E. Konofagou, T. Krouskop, R. Righetti and T. Varghese, "Elastographic Imaging," Ultrasound in Medicine and Biology, vo. 26, supplement 1, pp s23-s29, 2000.

[13] R. Muthupillai, P. J. Rossman, D. J. Lomas, J. F. Greenleaf, S. J. Riederer and R. L. Ehman, "Magnetic Resonance Imaging of Transverse Acoustic Strain Waves," Magnetic Resonance in Medicine, vol. 36, pp. 266-274, 1996.

[14] J. Ophir, I. Cespedes, B. Garra, H. Ponnekanti, Y. Huang and N. Maklad, "Elastography: Ultrasonic Imaging of Tissue Strain and Elastic Modulus In-vivo," European Journal of Ultrasound, vol. 3, pp. 49-70, 1996.

[15] M. O'Donnell, A. R. Skovoroda, B. M. Shapo, and S. Y. Emelianov, "Internal displacement and strain imaging using ultrasonic speckle tracking,", IEEE Transaction on Ultrasonics, Ferroelectrics and Frequency Control, vol. 41, no. 3, pp. 314-325, 1994.

[16] R. Zahiri-Azar and S. E. Salcudean, "Motion Estimation in Ultrasound Images Using Time Domain Cross Correlation with Prior Estimates," IEEE Transactions on Biomedical Engineering, vol. 53, no. 10, pp. 1990-2000, 2006.

[17] K. Zell, J.I. Sperl, M.W. Vogel, R. Neissner, and C. Haisch, "Acoustical Properties of Selected Tissue Phantom Materials for Ultrasound Imaging," Physics in Medicine and Biology, vol. 52, pp. 475-484, 2007.

[18] A. Goldstein, "The Effect of Acoustic Velocity on Phantom Measurements," Ultrasound in Medicine and Biology, vol. 26, no. 7, pp. 1133-1143, 2000.

[19] J. Ammann and B. Galaz, "Sound Velocity Determination in Gel-based Emulsions," Ultrasonics, vol. 41, pp. 569-579, 2003.

[20] F. A. Duck, A.C. Baker, and H.C. Starritt, Ultrasound in Medicine, Institute of Physics Publishing, Bristol, UK, 1998.

[21] T. Hall, M. Bilgen, M. F. Insana, and T. A. Krouskop, "Phantom Materials for Elastography," IEEE Transactions on Ultrasonics, Ferroelectrics, and Frequency Control, vol. 44, no. 6, pp. 1355-1365, 1997.

[22] M.O. Culjat, D. Goldenberg, P. Tewari and R.S. Singh, "A Review of Tissue Substitutes for Ultrasound Imaging," Ultrasound in Medicine and Biology, vol. 36, no. 6, pp. 861-873, 2010.

[23] J. Silver, Y. Ono and A. Adler, "Development of an Ultrasound Based System for Measuring Skeletal Muscle Motion," Carleton University, Canada, 2009. 
[24] J. Silver, Y. Ono, and A. Adler, "An ultrasonic technique for imaging of tissue motion due to muscle contraction," presented at IEEE Ultrasonics Symp., Rome, Italy, Sept. 21-23, 2009.

[25] W. Kroebel and K.H. Mahrt, "Recent Results of Absolute Sound Velocity Measurements in Pure Water and Sea Water at Atmospheric Pressure," Acoustica, vol. 35, pp. 154-164, 1976.

[26] Y. C. Fung, Biomechanics: Mechanical Properties of Living Tissues, NY, USA: Springer-Verlag, 1993.

[27] M. Shridhar, J. Liu, M. F. Insana, 'Viscoelasticity Imaging using Ultrasound: Parameters and Error Analysis," Physics in Medicine and Biology, vol. 52, pp. 2425-2443, 2007.

[28] J.D.N. Cheeke, Fundamentals and Applications of Ultrasonic Waves, Florida, USA: CRC Press, Appendix B, 2002.

[29] J. Ophir, "Elastography: A Quantitative Method for Imaging the Elasticity of Biological Tissues," Ultrasonic Imaging, vol. 13, pp. 111-134, 1991.

[30] R. S. C. Cobbold, "Foundations of Biomedical Ultrasound," New York, NY, USA: Oxford University Press, 2007.

[31] G. M. Artmann and S. Chien, "Bioengineering in Cell and Tissue Research," Berlin Heidelberg : Springer Berlin Heidelberg, 2008.

[32] T. Varghese and J. Ophir, "A Theoretical Framework for Performance Characterization of Elastography: The Strain Filter," IEEE Transactions on Ultrasonics, Ferroelectrics, and Frequency Control, vol. 44, no. 1, pp. 164-172, 1997.

[33] R. Righetti, J. Ophir and P. Ktonas, "Axial Resolution in Elastography," Ultrasound in Medicine and Biology, vol. 28, pp. 101-113, 2002.

[34] H. Chen, H. Shi and T. Varghese, "Improvement of Elastographic Displacement Estimation using a Two-step Cross-correlation Method," Ultrasound in Medicine and Biology, vol. 33, pp. 48-56, 2007.

[35] T. Varghese, J. Ophir and I. Cespedes, "Noise Reduction in Elastograms using Temporal Stretching with Multicompression Averaging," Ultrasound in Medicine and Biology, vol. 22, pp. 1043-1052, 1996. 
[36] R. Chanrasekhar, J. Ophir , T. Krouskop and K. Ophir, "Elastographic Image Quality vs. Tissue Motion In-vivo," Ultrasound in Medicine and Biology, vol. 6, pp. 847-855, 2006.

[37] H. Hisashi, S. Sasaki, M. Morimoto, K. Kawabata, E. Ito, K. Abe and T. Sambongi, "Imaging Elastic Properties of Soft Materials Immersed in Water using Force Modulated Mode in Atomic Force Microscopy," Journal of Applied Physics, vol. 37, pp. 3860-3863, 1998.

[38] D. A. Christensen, Ultrasonic Bioinstrumentation, NY, U.S.A: John Wiley \& Sons, 1988.

[39] J. Suri, C. Kathuria, R. Chang, F. Molainari and A. Fenster, Advances in Diagnostic and Therapeutic Ultrasound Imaging, Norwood, MA, USA: Artech House, 2008.

[40] M. Singh, H.R. Singh, and A. Kumar, "Development of Tissue-equivalent Phantoms for Biomedical Ultrasonic Applications," International Journal of Biomedical Engineering and Technology, vol. 1, no. 3, pp. 273-286, 2008. 